\section{A) Check for updates}

Cite this: Nanoscale, 2021, 13, 10668

\title{
A review of oligo(arylene ethynylene) derivatives in molecular junctions
}

\author{
Luke J. O'Driscoll (D) * and Martin R. Bryce (ID)*
}

Oligo(arylene ethynylene) (OAE) derivatives are the "workhorse" molecules of molecular electronics. Their ease of synthesis and flexibility of functionalisation mean that a diverse array of OAE molecular wires have been designed, synthesised and studied theoretically and experimentally in molecular junctions using both single-molecule and ensemble methods. This review summarises the breadth of molecular designs that have been investigated with emphasis on structure-property relationships with respect to the electronic conductance of OAEs. The factors considered include molecular length, connectivity, conjugation, (anti)aromaticity, heteroatom effects and quantum interference (QI). Growing interest in the thermoelectric properties of OAE derivatives, which are expected to be at the forefront of research into organic thermoelectric devices, is also explored.

\section{Introduction}

Oligo(arylene ethynylene)s (OAEs) consist of (hetero)aromatic ring systems linked by alkynes in an alternating fashion (Fig. 1a). The prototypical OAE is oligo(phenylene ethynylene)

Department of Chemistry, Durham University, Lower Mountjoy, Stockton Road, Durham, UK, DH13LE.E-mail: luke.j.odriscoll@durham.ac.uk, m.r.bryce@durham.ac.uk

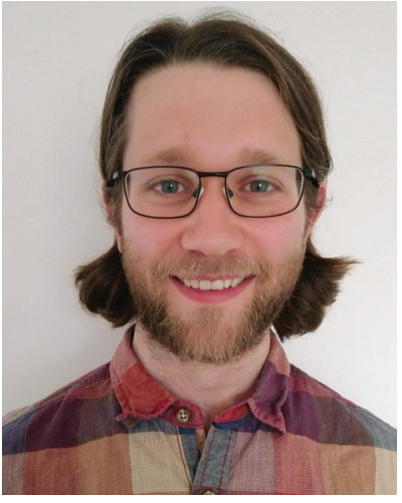

Luke J. O’Driscoll

molecules for molecular applications.
(OPE) (Fig. 1b). OAEs have found applications in diverse fields, including biosensors and biocides ${ }^{1}$ (including recently against the SARS-CoV-2 virus ${ }^{2}$ ), supramolecular chemistry ${ }^{3-5}$ and sensors, ${ }^{6,7}$ covalent organic frameworks, ${ }^{8}$ organic photovoltaics, ${ }^{9}$ and liquid crystals. ${ }^{10}$ Synthetically, OAEs have been used as graphene nanoribbon precursors ${ }^{11}$ and OAE templates have been used to prepare OAE macrocycles. ${ }^{12}$ OAEs have even been applied to the design of anthropomorphic molecules. ${ }^{13}$ This review focuses on OAEs designed for use in molecular

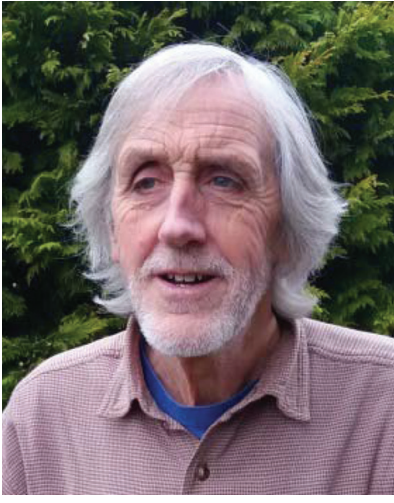

Martin R. Bryce
Martin Bryce graduated from Wolverhampton Polytechnic and obtained a D.Phil. from the University of York, UK. Following postdoctoral work at the University of British Columbia, Vancouver, with Prof. Larry Weiler, and at Bristol University with Prof. Roger Alder, Martin moved to Durham University. Since 1995 he has been a full professor of chemistry at Durham. He has held visiting scientist positions at the thesis of functional organic electronics and thermoelectric University of California, Santa Barbara, the University of Copenhagen and the University of Florida, Gainesville. Martin's research covers the design, synthesis and properties of optoelectronic materials (small molecules, oligomers and polymers), molecular electronics and nano-scale chemistry. 
a)

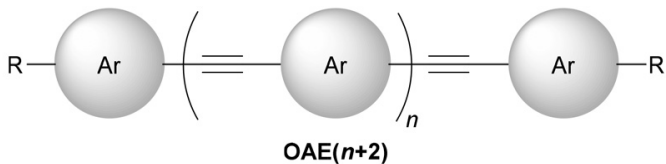

b)

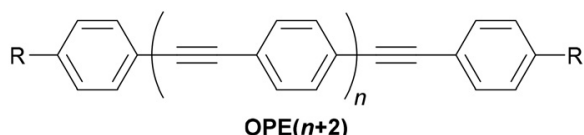

Fig. 1 (a) Illustration of a general $\mathrm{OAE}(\mathrm{OAE}(\boldsymbol{n}+2))$ with unspecified aryl units and terminal $\mathrm{R}$ groups. Each Ar or $\mathrm{R}$ group can be the same or different. (b) Structure of a general para-conjugated OPE $(\mathrm{OPE}(n+2))$ with terminal functionality ( $\mathrm{R}$ groups), which can be the same or different.

electronics applications, specifically in molecular junction experiments, where the OAE forms the conjugated, conductive backbone of a molecular wire held between two electrodes.

This review aims to highlight the chemical diversity of OAE derivatives and to discuss the impact of structural variation on their electronic and thermal properties in molecular junctions. Work in this field is highly interdisciplinary, combining chemical synthesis of bespoke molecules, experimental studies of their assembly and transport properties in junctions, and computational studies that provide insights into the experimental results. The topic is approached from a chemical viewpoint, but to provide appropriate context this introduction will include a concise overview of experimental and theoretical methods used in the study of molecular junctions.

\subsection{Nomenclature}

OAE molecular wires used in molecular junctions incorporate two essential structural features: (i) the OAE backbone itself and (ii) anchoring units to attach this backbone to electrodes (in some cases, anchoring groups also form part of the OAE backbone, e.g. terminal pyridine rings). A third feature that is often present is additional "side group" functionality, e.g. alkyl chains to enhance solubility. Section 2 provides a detailed discussion of the structural variation of OAEs.

This review will follow the usual convention in molecular electronics of naming OAEs (especially OPEs) based on the number of aromatic units present in the backbone. Hence when $n=1$, the molecule shown in Fig. $1 \mathrm{~b}$ is an OPE3. Shorter molecules, e.g. OAE2 derivatives (tolanes, $n=0$ ) are commonly studied. Studies of OPE series often include species based on a single benzene ring. They will be considered within this review where they serve as model compounds in this manner. Note that although benzene derivatives are sometimes referred to as OPE1 in this context, these species are not truly OPEs (they are not oligomers and do not contain ethynylene fragments).

Using this nomenclature, aromatic systems always form the termini of an OAE backbone; any additional functionality, even extra alkynes, will be considered either as anchoring groups or side groups, as appropriate. As discussed below, para-conjugated systems are favoured for high conductance and this review will consider this to be the default geometry; any deviation from all-para geometry will be emphasised. Note that it is common, but not necessary, for OAEs studied in molecular junctions to be symmetrical; this facilitates synthesis and prevents potential complexity regarding the orientation of molecules within a junction.

The scope of this review will extend to molecules in which arylene ethynylene backbones are used to link other functional groups, affording OAE-like molecular wires (see sections 2.2.4 and 2.2.5).

\subsection{Experimental and computational methods}

The conductance properties of molecular wires have been investigated using various methods. These are based on molecular junctions in which either single molecules or molecular assemblies are trapped between two electrodes. Single-molecule studies are the more common approach for OAEs, but numerous ensemble studies have also been reported, as will be seen below. Single-molecule conductance approaches include the scanning tunnelling microscopy break-junction method (STM-BJ), ${ }^{14}$ summarised in Fig. 2, and the mechanically controlled break junction (MCBJ) ${ }^{15}$ technique, summarised in Fig. 3. Ensemble studies of molecular assemblies, such as self-assembled monolayers (SAMs), can be carried out using
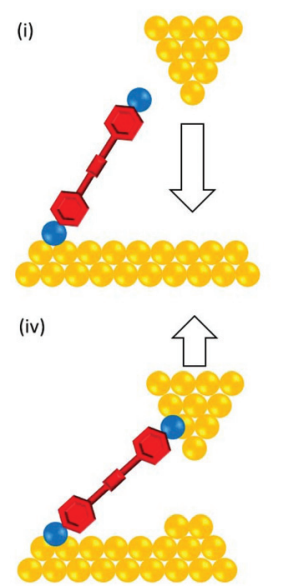

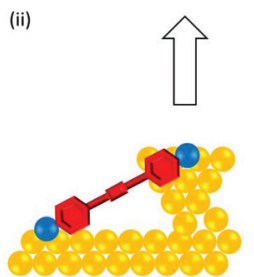

(v)

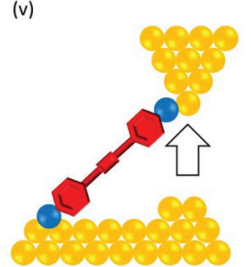

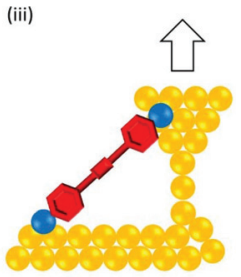

(vi)
Fig. 2 Cartoon illustrating an experimental cycle of the STM-BJ method with Au electrodes: (i) A Au STM tip is lowered towards a Au surface functionalised with the target molecule (or in a solution of the molecule). (ii) The tip contacts the surface and forms a junction containing multiple Au atoms. One or more molecules can bridge between the tip and the surface. Junction conductance is an integer multiple of $G_{0}$ (the conductance quantum). (iii) The tip is retracted until the junction contains a single gold atom. Junction conductance is $G_{0}$. (iv) Upon further retraction, the final $\mathrm{Au}-\mathrm{Au}$ contact ruptures and a molecule can remain bridging the junction. The molecular conductance is now detected (substantially less than $G_{0}$ ). (v) The geometry and conductance of the Au-molecule-Au junction vary slightly as the tip is retracted further. (vi) Further retraction results in the rupture of the molecular junction and the cycle can begin again. A typical experiment requires thousands of cycles. The Au surface can become disordered after repeated tip contacts; periodically changing the scan region can ensure good quality data is collected. 


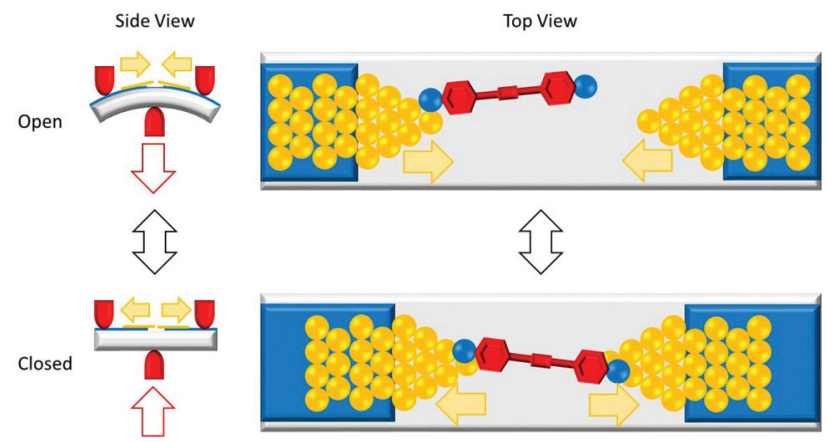

Fig. 3 Cartoon illustrating the MCBJ method. A piezoelectric motor is used to move push rods (red) to bend a flexible substrate (grey) with high precision. The substrate is coated with an insulating material (blue) upon which gold electrodes with sharp tips (yellow) are attached. The sharp tips required can be formed by breaking a single wire in situ. The electrodes are exposed to a solution of the target molecule. Lowering (raising) the central push rod closes (opens) the junction, decreasing (increasing) the distance between the tips. At sufficiently close proximity, a molecular junction can form, bridging the electrodes.

techniques including conductive probe atomic force microscopy (c-AFM) ${ }^{16}$ summarised in Fig. 4 , and eutectic gallium-indium (EGaIn) electrode based methods, ${ }^{17}$ summarised in Fig. 5. These and other methods have been thoroughly described in many reviews, ${ }^{18-26}$ so will not be subject to detailed discussion here. Some aspects of their development are discussed in section 1.3. Thermal properties of molecular wires can be determined using modifications to these and other methods. ${ }^{27-30}$

Molecular junctions are widely investigated using computational methods. Charge transport simulations using molecular orbitals derived from density functional theory (DFT) or Hückel (tight-binding) theory are used to calculate transmission functions for molecular junctions. ${ }^{25,31} \mathrm{~A}$ transmission function shows how the transmission coefficient $T(E)$ of a molecular junction varies with its energy $E$, usually considered relative to the system's Fermi energy $\left(E_{\mathrm{F}}\right)$. It represents the probability that an electron of energy $E$ will pass across a junction from one electrode to the other, through the molecule. $T(E)$ is proportional to conductance, $G$. In a typical molecular junction study, at low bias and in the absence of external influ-

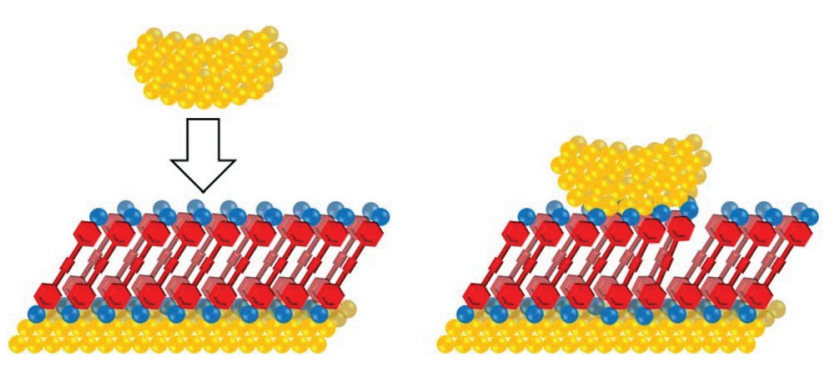

Fig. 4 Cartoon illustrating the C-AFM method. A c-AFM tip is used to contact a SAM of the molecule of interest on a Au surface. The large area of the tip relative to a molecule means multiple molecules bridge the surface-tip gap and contribute to junction conductance.

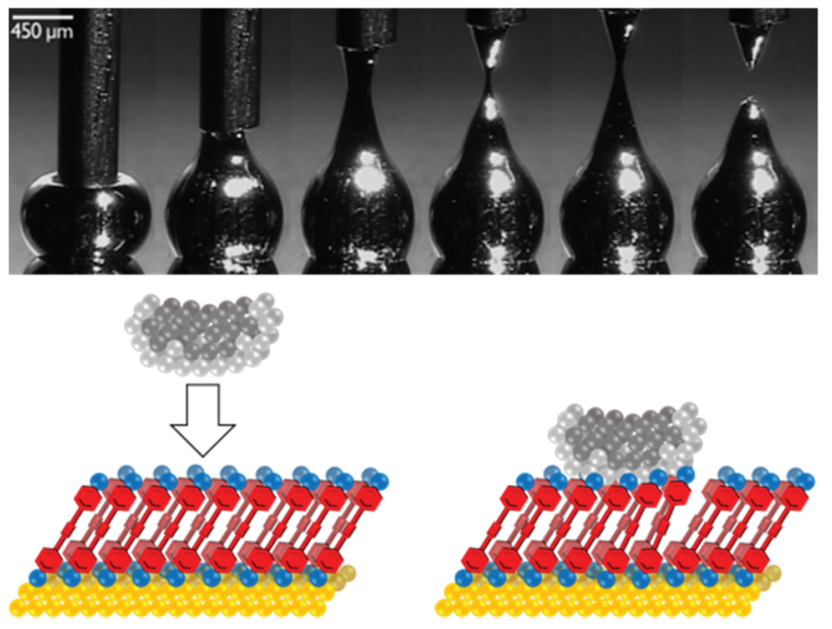

Fig. 5 Above: Sequential images showing formation of an EGaln tip by withdrawal of a syringe needle filled with EGaln from a drop of EGaln on a silver surface. This figure has been reproduced from ref. 17 with permission from Wiley-VCH, copyright 2008. Below: Cartoon of an EGaln tip contacting a SAM of molecules of interest on a Au surface. Lighter grey spheres represent the $\mathrm{Ga}_{2} \mathrm{O}_{3}$ oxide layer that coats the EGaln (darker grey spheres). The large area of the tip relative to a molecule means multiple molecules bridge the surface-tip gap and contribute to junction conductance.

ences such as gating, the energy of interest is usually close to $E_{\mathrm{F}}$. However, a disadvantage of charge transport simulations is that they often fail to provide accurate values of $E_{\mathrm{F}}{ }^{31,32}$ This can be mitigated through methods such as spectral adjustment, ${ }^{32}$ where energy levels are modified based on empirical data. Another source of discrepancy between theoretical and experimental studies is that charge transport simulations generally consider a single optimised junction geometry, whereas the geometry of an experimental junction is largely unknown, cannot be precisely controlled, and in the case of break junctions varies for each individual conductance trace. Nevertheless, at least qualitative agreement is usually seen with experimental conductance studies. ${ }^{25}$

Fig. 6 shows a representation of two typical transmission functions. Important features include resonances coincident with the highest occupied molecular orbital (HOMO) and lowest unoccupied molecular orbital (LUMO) of the molecule, which appear as Lorentzian peaks with maxima where $T(E)=$ 1. $E_{\mathrm{F}}$ lies in the region between these peaks, the HOMOLUMO gap. The orbital closest to $E_{\mathrm{F}}$ is most influential on the properties of the junction. ${ }^{33}$ Transmission functions contain features relating to quantum interference (QI) effects (see section 3.2). In most cases a smooth, featureless curve between the HOMO and LUMO peaks is indicative of constructive QI (CQI) (blue solid curve, Fig. 6). Destructive QI (DQI) is associated with sharp antiresonances where $T(E)$ approaches 0 (red dashed curve, Fig. 6). In simple systems these features occur close to $E_{\mathrm{F}}$ and result in low low-bias conductance. Certain structural features can cause antiresonances to be shifted away from $E_{\mathrm{F}}$ and thereby reduce their impact on conductance. ${ }^{34-36}$ 


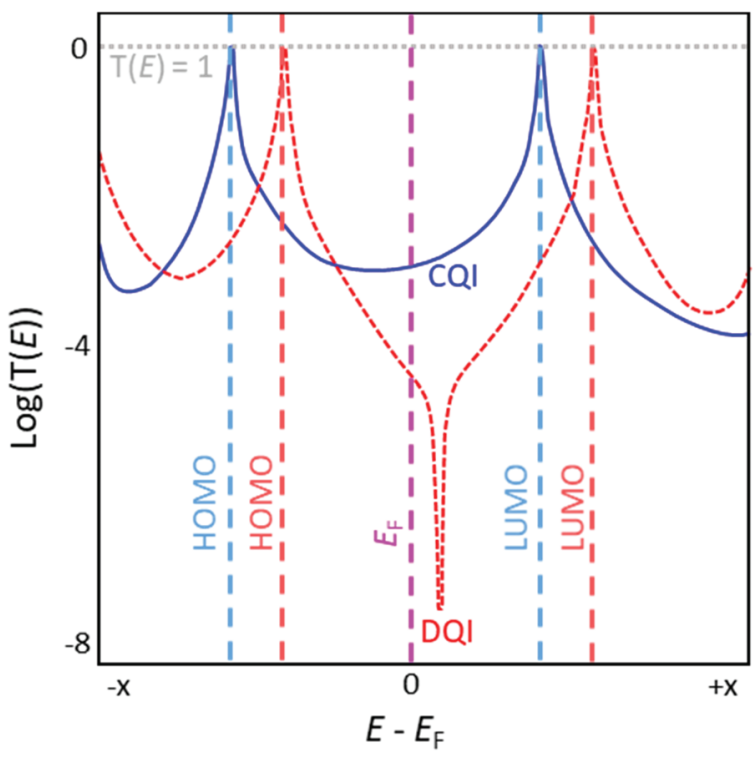

Fig. 6 Illustration of representative transmission functions of molecules exhibiting CQI (blue solid curve) and DQI (red dashed curve) on an arbitrary energy scale. Molecular orbital energies for each molecule are indicated using dashed lines in a matching colour, with the Fermi energy $\left(E_{\mathrm{F}}\right)$ shown in pink. This figure has been adapted from ref. 34 with permission from The Royal Society of Chemistry, copyright 2021.

\subsection{A history of OAEs in molecular electronics}

In the $1990 \mathrm{~s}$, before the technology to measure the conductance of molecules in junctions was developed, Tour and colleagues advocated OAEs as ideal candidates for conjugated molecular wires, and extensively described their synthesis and assembly on surfaces. ${ }^{37-43}$

The earliest example of an OAE wire (indeed, any conjugated molecular wire) in a molecular junction was presented in the 1996 work of Bumm et al., ${ }^{44}$ entitled "Are single molecular wires conducting?" The OPE3 molecule 1 (Fig. 7), prepared in Tour's group, ${ }^{37}$ was embedded within a SAM of $n$-dodecanethiol on a $\mathrm{Au}(111)$ surface. An STM was used to locate molecules of $\mathbf{1}$ within the SAM and to show that the conductance of 1 appeared qualitatively higher than the surrounding $n$-dodecanethiol, despite 1 being a longer molecule. Subsequently, an approach was developed in which the application of voltage pulses allowed patterning of OPE wires within an $n$-dodecanethiol matrix. ${ }^{45}$

After establishing that conjugated OAE-type molecular wires were, as expected, more conductive than saturated alkanethiols, molecular conductance was probed in a quantitative manner. Methods used to achieve this can be broadly divided

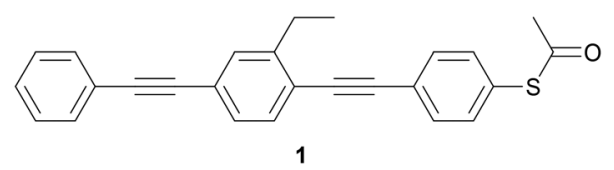

Fig. 7 OPE3 molecule 1 which was used in the earliest study of an OAE in a molecular junction. into those based on nanoscale molecular junctions containing individual molecules, and those based on larger molecular junctions containing many molecules. Single-molecule methods mostly rely on repeated junction formation, measurement and breaking in order to acquire statistically significant data sets, usually comprising hundreds or thousands of individual conductance traces. ${ }^{46}$ Advances in methodology mean this process can now largely be automated. In the vast majority of cases single-molecule measurements use $\mathrm{Au}-$ molecule-Au junctions. Ensemble approaches measure many molecules simultaneously and permit easier variability of electrode materials. However, determining "per molecule" contributions is not straightforward, which can complicate quantitative comparisons between studies or dissimilar molecules.

1.3.1. Electrical conductance. In 1997, Reed et al. determined the molecular conductance of benzene-1,4-dithiol in the first single-molecule MCBJ study. ${ }^{15}$ By 2002, the MCBJ method (Fig. 3) had been applied to OPE3 species. ${ }^{47}$ The STM-BJ technique (Fig. 2) was first reported by Xu and Tao in $2003,{ }^{14}$ in a study of alkanedithiols and 4,4'-bipyridine. The method was applied to benzene-1,4-dithiol ${ }^{48}$ and OPE3 dithiol species ${ }^{49}$ in the following two years. Both methods have since been widely adopted for the investigation of OAEs and other molecular wires. Variations on the STM-BJ approach include the $I(s)^{50}$ and $I(t)^{51}$ methods, which both avoid contact between the STM tip and underlying electrode and thus are particularly suited to delicate analyte assemblies. These techniques can also reduce the number of conductance features observed, ${ }^{52}$ facilitating data analysis, but they are more time consuming than standard STM-BJ methods as they require spontaneous junction formation.

c-AFM was developed to study the dielectric properties of $\mathrm{SiO}_{2}$ films in the early $1990 \mathrm{~s},{ }^{53}$ but was later applied to molecular conductance studies. By the early $21^{\text {st }}$ century c-AFM had been used to investigate the conductance of alkanethiols ${ }^{16,54}$ and conjugated molecules, ${ }^{54}$ including OAEs. ${ }^{55}$ By using mixed monolayers in some cases a single-molecule junction can be created in a cAFM experiment. ${ }^{55}$ Single-component SAMs are more common for OAEs; here the number of molecules in a c-AFM junction can vary from tens to hundreds. Ideally, the same AFM tip and force are used for a series of related molecules to ensure a similar contact area between the tip and the molecular assembly. Assuming the molecules assemble with similar density, the number of molecules contacted should be similar across the series, ${ }^{56}$ allowing valid comparisons to be made. To compare dissimilar molecules, the Hertz model ${ }^{57-59}$ can be used to approximate the contact area of the c-AFM junction. In conjunction with methods to determine molecular area, such as reductive desorption, the conductance per molecule can then be estimated. ${ }^{60}$ While break junction methods rely on identical electrode materials, c-AFM can investigate asymmetric junctions, for example by contacting assemblies of molecules on gold using AFM tips made of (or coated with) Ag, ${ }^{56} \mathrm{Pt}^{56,60}$ or graphene. ${ }^{60}$ As the tip is pushed into contact with the SAM, specific anchoring groups are not necessary at the top contact. 
The EGaIn method (Fig. 5) was first reported by Chiechi et al. in $2007 .{ }^{17}$ It has several advantages over the similar, older Hg-drop electrode method, ${ }^{21,61}$ not least the avoidance of toxic mercury which can form undesired amalgams with other metallic electrodes. ${ }^{17}$ EGaIn is a room temperature liquid metal from which a sharp tip can be formed by pulling a syringe of EGaIn away from a drop (Fig. 5). The tip retains its shape due to a surface layer of $\mathrm{Ga}_{2} \mathrm{O}_{3}$, which imparts the liquid with non-Newtonian properties. ${ }^{21}$ It can conform to a SAM surface upon contact. An EGaIn junction is therefore of the form: bottom electrode-SAM- $\mathrm{Ga}_{2} \mathrm{O}_{3}$-EGaIn, where the bottom electrode can be e.g. $\mathrm{Au}^{62} \mathrm{Ag}^{17}$ or $\mathrm{Pt} .{ }^{63}$ The tip diameter is largely defined by the properties of EGaIn, meaning it is consistently $c a .25 \mu \mathrm{m} .^{21}$ Interactions between the SAM and $\mathrm{Ga}_{2} \mathrm{O}_{3}$ layer are generally non-covalent, meaning that molecules do not necessarily require an anchoring group to interact with the top contact. As for c-AFM, it is possible to estimate the effective contact area of the EGaIn electrode to facilitate comparison with other methods. ${ }^{64}$ The conductance of an OAE was investigated using EGaIn in 2011, ${ }^{65}$ and data for a series of OPEs were presented in $2016 .{ }^{62}$ Subsequent studies examined more complex OAEs. ${ }^{63,66-69}$

An older method for determining ensemble conductance that is rare in recent literature is a crossed-wire junction. ${ }^{70} \mathrm{~A}$ SAM assembled on a Au wire is contacted by a second perpendicular Au wire, forming a junction containing ca. 1000 molecules. Early examples of this technique used OPE3s, amongst other molecules. $^{70}$

A third family of conductance studies are those based on nanogaps. The approaches used are much more variable between research groups than e.g. break junction studies. In general, they rely on the fabrication of devices in which two electrodes are separated by a nanoscale distance. Electrode materials can include gold, ${ }^{71}$ silicon, $^{72}$ graphene $^{73-75}$ and carbon nanotubes (CNTs). ${ }^{76,77}$ When exposed to a solution of a molecular wire of suitable length, it is possible for the gap to be bridged by one or more molecules which bind to both sides. The electronic properties of the junction can then be investigated. While the number of bridging molecules can vary between devices and depend on the nature of the nanogap, it is possible to achieve junctions containing a single molecule. ${ }^{73}$ However, the yield of suitable junctions is frequently low, ${ }^{71,73}$ prohibiting the collection of large data sets common to break junction methods.

Molecular junctions are not the only method by which charge transport along a molecular backbone can be investigated. $^{78}$ OAE derivatives also find use as bridging units between functional groups in donor-bridge-acceptor (D-B-A) systems, ${ }^{79-81}$ mixed valence complexes $^{82,83}$ and biradical systems. ${ }^{84}$ Such studies are outside the scope of this review.

1.3.2 Thermoelectric properties. In 2007, Reddy et al. used a modified STM-BJ approach to determine the Seebeck coefficient (thermopower) of single-molecule junctions containing molecules including benzene-1,4-dithiol, ${ }^{85}$ opening the door to the study of the thermoelectric properties of single molecules. Venkataraman and co-workers developed a method to simultaneously measure the conductance and Seebeck coefficient of molecular junctions in $2012,{ }^{86}$ reporting data for molecules including an OPE2 diamine. A modified MCBJ was used to measure the Seebeck coefficient of benzene-1,4-dithiol in 2015. ${ }^{87}$ However, single-molecule thermal conductance measurements were not achieved until 2019, when two STM-BJ based methods were independently reported by Reddy and colleagues, ${ }^{88}$ who measured a series of alkanedithiols, and by Mosso et al., ${ }^{89}$ who studied an alkanedithiol and an OPEdithiol. Now that the technology to measure the electronic conductance $(G)$, Seebeck coefficient $(S)$ and thermal conductance $(\kappa)$ of individual molecules is in place, it is possible to experimentally determine the thermoelectric figure of merit $(Z T)$ for molecular wires and identify promising candidates for organic thermoelectric devices. More detail can be found in section 4, which focuses of the thermoelectric properties of OAEs in junctions.

Ensemble approaches can also be used to analyse the thermal properties of molecules. Using c-AFM it is possible to simultaneously measure electrical conductance and thermopower. ${ }^{90}$ Measurement of thermopower using an EGaIn electrode was reported in $2018 .^{91}$ Notably, the Seebeck coefficient of a molecular junction is independent of the number of molecules present. ${ }^{28}$ This means that unlike studies of electrical conductance, comparisons can be made between materials and methods without needing to estimate the number of molecules present in an ensemble junction. Thermal conductance measurements of SAMs are possible, but so far are largely limited to alkane species. ${ }^{27}$

\subsection{Why use OAEs for molecular electronics?}

As the field of molecular electronics has developed, many different conductive conjugated backbones have been utilised in molecular wires. These include oligo(arylene vinylene)s ${ }^{92}$ (most commonly oligo(phenylene vinylene) (OPV) ${ }^{93-95}$ ), oligoarylenes $^{96}$ (including oligophenylenes $(\mathrm{OP})^{33,54,97,98}$ and oligothiophenes (OT) $)^{99-101}$ ), oligoynes (OY), ${ }^{102,103}$ cumulenes $(\mathrm{CM})^{104}$ and porphyrin tapes $(\mathrm{PT}){ }^{105}$ for which generic examples are shown in Fig. 8. Various ladder compounds have also been used as backbones; Fig. 8 shows representative examples of a ladder fluorene (LF), ${ }^{106}$ carbon-bridged OPV (CBO), ${ }^{71}$ and heteroacene (HA). ${ }^{107}$ Nevertheless, OAEs continue to attract considerable attention. In particular, OPE3-dithiol (Fig. 1b, $n=1, \mathrm{R}=\mathrm{SH}$ ) has been described as "a "fruit fly" molecule for detailed single-molecule transport studies'. ${ }^{108}$ Some key advantages of OAEs relative to alternative backbones include:

- Rigidity. OAEs are inflexible and length-persistent due to the sp-hybridised carbons within the alkyne units (unless multiple non-para-connectivities are present). While OPVs have been shown to be more conductive than OPEs, ${ }^{93}$ the possibility for isomerisation about alkene bonds means significant conductance variation is observed for OPVs. ${ }^{94}$ The possibility of aryl-aryl bond rotation means that oligothiophenes are not length-persistent; this isomerism has also been shown to affect conductance. ${ }^{100}$ 
<smiles>[R]c1ccc(C=Cc2ccc(C=Cc3ccc([R])cc3)cc2)cc1</smiles>

OPV

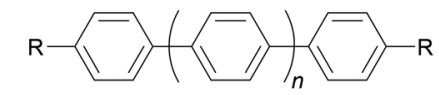<smiles>[R]c1ccc(C(C)(C)c2ccc(C(C)(C)c3ccc([R])s3)s2)s1</smiles>

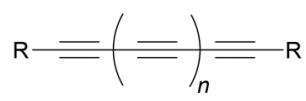

$\mathrm{R}_{\mathrm{R}}^{\mathrm{R}^{\prime}}=\bullet \neq \cdot \neq_{\mathrm{R}^{\prime}}^{\mathrm{R}}$

OY

$\mathrm{CM}$

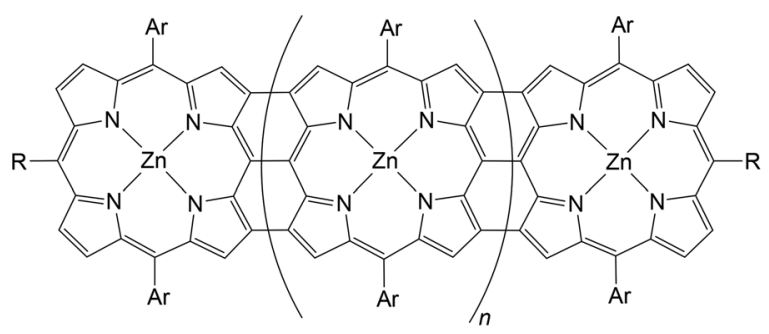

PT

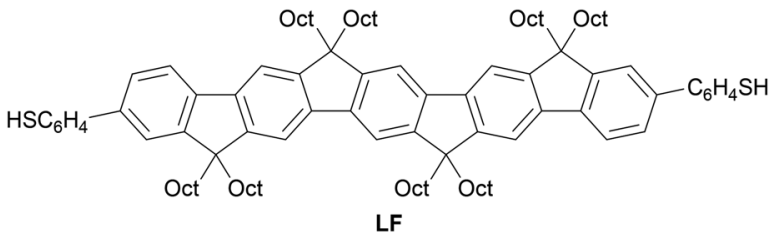
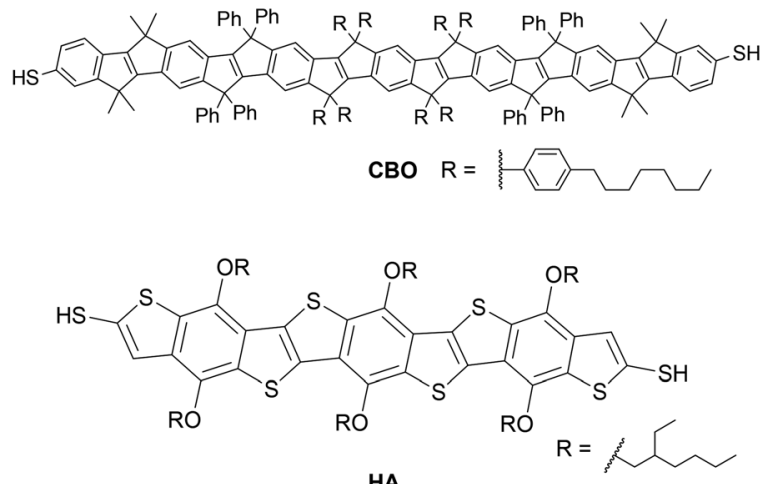

HA

Fig. 8 Structures of alternative conjugated backbones used in molecular wires.

- Stability. Oligoynes and cumulenes are rigid systems but beyond relatively short molecular lengths they are unstable in the absence of significant steric shielding. ${ }^{102,104}$ OAEs benefit from the rigidity of alkyne bonds but do not suffer from the same stability concerns.

- Synthetic accessibility and variability. Other rigid, stable backbones such as ladder compounds, ${ }^{71,106,107}$ butadiynyl- linked porphyrins ${ }^{109}$ and porphyrin tapes ${ }^{105}$ exhibit favourable conductance properties. However, their widespread uptake is hindered by synthetic challenges. In contrast, protocols for the preparation of OAE wires are well-established, typically highyielding, and readily allow the incorporation of diverse functionality, as discussed in section 2.

- Minimal steric strain. Oligophenylene wires are rigid, stable and readily synthesised, but their backbones are necessarily twisted due to steric strain between consecutive rings. Conductance has been shown to be a function of the dihedral angle between adjoining rings, and is highest for coplanar systems. ${ }^{110}$ The presence of an ethynyl unit between each aryl ring in OAEs means that all the aryl rings can be near-coplanar, although there is a low barrier to rotation of the aryl rings. ${ }^{111}$ OAE wires are, therefore, more favourable than oligophenylenes. While not necessarily representative of junction configurations, X-ray crystal structures of OAEs of different lengths show that near-coplanarity of aryl rings is often favoured in the solid state. ${ }^{112}$

- Magnitude of conductance. OAEs are experimentally convenient as the conductance of molecules from OAE2 to OAE5 and beyond generally lies within the window accessible to common measurement techniques. At shorter lengths, up to ca. OAE3, species where conductance is reduced, for example due to QI, are also often within the experimentally accessible range. This means that series of OAEs can provide insightful studies of structure-property relationships and QI effects. Many of these studies are discussed in section 3.

\section{Structure and synthesis of OAEs for molecular junctions}

This section will summarise some key synthetic methods used to prepare OAE molecular wires then highlight the structural variations possible within the OAE framework.

\subsection{Synthetic methods}

The Sonogashira cross-coupling reaction ${ }^{113,114}$ has been fundamental to OAE synthesis since the early days of the field. ${ }^{40}$ Indeed, a detailed review from 2011 remains highly relevant. ${ }^{115}$ An example of OAE synthesis using the Sonogashira coupling is shown in Scheme 1a and a general catalytic cycle is shown in Scheme 1b. It should be noted that uncertainty remains over the exact nature of the Cu-catalysed cycle. ${ }^{113-115}$ A palladium- and copper-catalysed reaction takes place between an aryl halide (or pseudohalide such as triflate, -OTf, or diazonium salt, $-\mathrm{N}_{2} \mathrm{X}$ ), Ar-X, and a terminal alkyne, $\mathrm{H}-\mathrm{C} \equiv \mathrm{C}-\mathrm{R}$, in which a $\mathrm{C}-\mathrm{C}$ bond is formed between the (pseudo)halidebearing $\mathrm{sp}^{2}$ carbon of the aryl unit and the proton-bearing $\mathrm{sp}$ carbon of the acetylene, forming $\mathrm{Ar}-\mathrm{C} \equiv \mathrm{C}-\mathrm{R}$ with effective loss of HX. Iodides are the most reactive halide, although triflates and bromides suffice in many cases. Mixed-halide precursors can be used chemoselectively in the synthesis of asymmetric systems. ${ }^{115}$ The presence of electron withdrawing groups on the aryl halide also enhances reactivity. ${ }^{115}$ 
a)

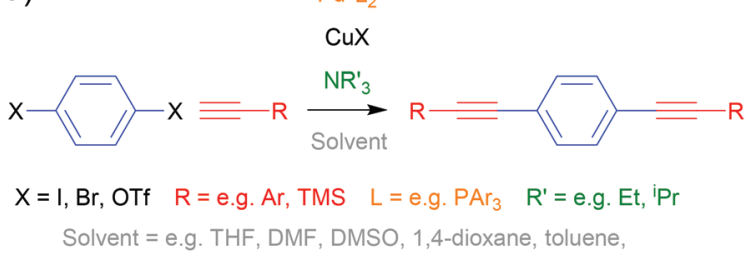

b)

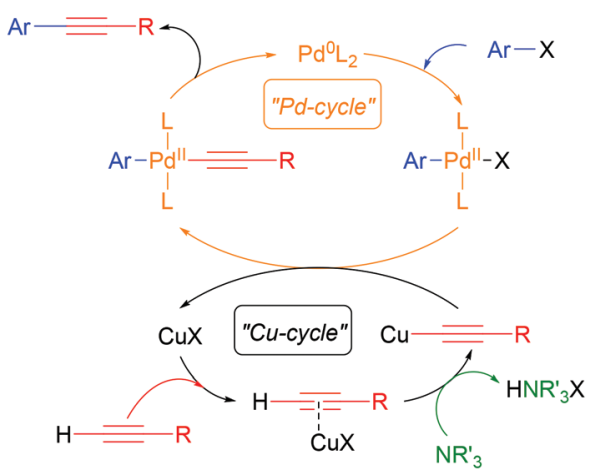

Scheme 1 Generic reaction (a) and general catalytic cycle (b) for the Sonogashira coupling as used in the preparation of OAEs and OAE precursors. For brevity, cis/trans-isomerisation of the Pd-complexes is not shown, although it should be noted that cis-geometry is required for the reductive elimination step.

Extensive early synthetic work by the Tour group on long OAEs utilised solid-supported synthesis. ${ }^{40}$ This method typically afforded a series of compounds with doubling lengths, e.g. OAE2, OAE4, OAE8, OAE16. Solid-supported approaches are rare in more recent literature, in which shorter OAEs are usually preferred in order to achieve experimentally-detectable conductances. For this reason smaller length differentials are usually desirable: the conductance of an OAE2, OAE3, OAE4 series is much less challenging to determine experimentally than that of an OAE2, OAE4, OAE8 series. For the typical length range of OAEs, at least at research laboratory scale, solution-based synthesis is currently favoured.

The broad availability of suitable aryl halide precursors and monoprotected alkynes such as trimethylsilylacetylene (TMSA) means that many OAE backbones can be readily synthesised in a stepwise fashion. For example, an OAE3 system can be prepared from a dihalogenated arene by first performing a twofold Sonogashira coupling with TMSA to afford a protected diyne (Scheme 1a, R = TMS). After deprotection, another twofold Sonogashira coupling with a halogenated anchoring group would afford an OAE3. Alternatively, the halogenated anchoring group could be functionalised with TMSA, deprotected and then coupled to the dihaloarene (Scheme 1a, $\mathrm{R}=$ anchoring group). Alternative methods ${ }^{115}$ to prepare alkyne precursors include the Corey-Fuchs synthesis, which requires an aldehyde precursor; however, this protocol is rarely utilised in the preparation of OAE wires for molecular junctions.

Not all key building blocks for OAE wires are available commercially. The functionalisation or construction of more complex polycyclic systems can often be necessary prior to their use in Sonogashira couplings, and can require many synthetic steps. ${ }^{66,116,117}$ This can be particularly relevant for connectivity in unreactive positions, even for simple monocyclic species. ${ }^{118}$

As an example, the key building block 4-(acetylthio)iodobenzene (2) will be considered. Although acetyl-protected thiols are arguably the most popular anchoring unit for OAE wires, 2 is not widely available commercially. However, it can be prepared from various precursor molecules. Scheme 2 shows the following synthetic protocols for 2: (a) nucleophilic substitution of a diazonium salt ${ }^{119}$ (prepared from 4-iodoaniline, not shown), (b) reduction and protection of 4-iodobenzenesulfonyl chloride, ${ }^{120}$ (c) a multi-step transformation of 4-iodophenol, ${ }^{121}$ and monosubstitution of 1,4-diiodobenzene using either (d) butyllithium ${ }^{42}$ or (e) copper catalysis. ${ }^{60,122}$ To our knowledge, the diazonium route was the first reported synthesis of 2, but it is not frequently used. The reduction method is more widespread and reportedly high-yielding. This route and the multi-step route from 4-iodophenol are both described as more reliable than selective monolithiation. ${ }^{120,121}$ The copper-catalysed route was reported more recently. ${ }^{122}$ It relies on statistical monosubstitution, but this can be favoured by using an excess of diiodide and recycling the unreacted material. ${ }^{60}$

The relatively labile nature of the acetyl protecting group means that it is not compatible with basic reaction conditions, and consequently 2 must often be added in the final synthetic step. Where this is not possible or convenient, transprotection of other sulfur species (e.g. tert-butyl or cyanoethyl thioethers) provides an alternative. ${ }^{123-126}$ These less labile protecting groups also find use in approaches reliant on orthogonal protection. For example, cyanoethyl thioethers were used in conjunction with 2-hydroxyprop-2-yl protected alkynes in the synthesis of long OAEs, in which the polar 2-hydroxyprop-2-yl protecting group additionally facilitated purification. ${ }^{127}$ Pyridine, amine and methyl thioether (SMe) anchors have a wider tolerance of reaction conditions than thioacetates, and halogenated

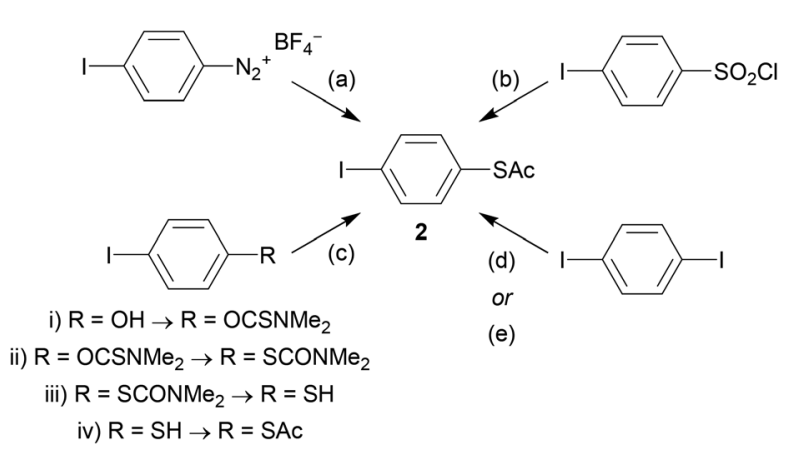

Scheme 2 Synthetic routes to 4-(acetylthio)iodobenzene (2). Reagents and conditions: (a) KSAc, DMSO, RT; (b) i. Zn, $\mathrm{Me}_{2} \mathrm{SiCl}_{2}, 1,2$-dichloroethane, DMA, $75{ }^{\circ} \mathrm{C}$, ii. $\mathrm{AcCl}, 50{ }^{\circ} \mathrm{C}$; (c) i. $\mathrm{NaH}, \mathrm{N}, \mathrm{N}$-dimethylthiocarbamoyl chloride, DMF, RT $\rightarrow 80^{\circ} \mathrm{C}$, ii. $235^{\circ} \mathrm{C}$, in melt, iii. $\mathrm{KOH}$, $\mathrm{MeOH}$, reflux, iv. $\mathrm{AcCl}$, pyridine, $\mathrm{RT}$; (d) i. $t$ - $\mathrm{BuLi}^{\circ} \mathrm{Et}_{2} \mathrm{O},-78^{\circ} \mathrm{C}$, ii. $\mathrm{S}_{8}, \mathrm{THF}$, $0{ }^{\circ} \mathrm{C}$, iii. $\mathrm{AcCl},-78{ }^{\circ} \mathrm{C}$; (e) $\mathrm{KSAC}$, Cul, 1,10-phenanthroline, toluene, $100{ }^{\circ} \mathrm{C}$ or reflux. 
derivatives are readily available. These anchor groups can, therefore, be utilised earlier in a synthetic route without the need to prepare them first. This helps to rationalise the widespread use of these anchoring groups despite the oftensuperior properties of thiols - e.g. stronger binding to $\mathrm{Au}$ and higher conductance.

\subsection{Structural diversity of OAEs}

As stated above, an OAE wire can be considered to consist of two primary components: the conductive OAE backbone and its anchoring groups. Side group functionalisation of the OAE backbone is another common feature. Variation based on each of these three structural components will be discussed below. The use of OAE backbones as a means of incorporating other functionality into molecular wires will then be examined.

2.2.1 Conductive backbone. Three elements within an OAE backbone can be easily varied synthetically to investigate structure-property relationships in molecular wires. These are: (1) oligomer length, i.e. the number of arylene-ethynylene repeat units, (2) the nature of the aryl units, which can vary from simple benzene rings to complex polycyclic systems such as porphyrins, and (3) geometry, i.e. the connectivity between aryl units and alkynes (e.g. ortho, meta, or para for 6-membered rings).

Fig. 9 shows OAEs 3-6 which have long backbones (up to OAE9) and have been studied in molecular junctions. ${ }^{76,112,128,129}$ From a synthetic viewpoint, while the preparation of shorter OAEs is less laborious, molecular length is limited primarily by availability and solubility of precursors. Given these factors, increasingly long wires can be prepared iteratively by increasing the number of synthetic steps performed. Indeed, early work on OAE wires describes the synthesis of an OPE18, ${ }^{41}$ OAE19 ${ }^{42}$ and OAE23. ${ }^{43}$ The discrepancy between the lengths of OAE available synthetically and those typically measured in junctions in part relates to the decay of conductance with molecular length (see section 3.1); the electrical conductance of very long OAEs is generally below the noise level of the equipment used to determine molecular conductance experimentally. Longer molecules are necessarily more prone to intermolecular interactions than shorter analogues, and surface interactions, through e.g. van der Waals forces, can effect molecular assembly. ${ }^{130}$ There is no clearly defined limit on the molecular length for which measurements are practical, as conductance properties are influenced by many other factors. However, most conductance studies investigate OAE5 molecules or shorter, as will be seen in the discussion below. This range coincides with OAEs which can be synthesised relatively quickly and easily.

The number of OAE repeat units is not the only factor to affect molecular wire length. Clearly, the size of arylene systems can have a significant influence (e.g. benzene vs. porphyrin, or OAE9s 4 and 5 in Fig. 9). Changes to molecular geometry exert little effect on the length of the conductance pathway through the OAE backbone in terms of the number of $\mathrm{C}-\mathrm{C}$ bonds included. However, they can significantly alter the shortest through-space distance between anchoring groups and hence reduce the distance between junction electrodes.

Many different aryl groups have been incorporated into conductive OAEs. The effect on electronic conductance of factors such as the connectivity, aromaticity and extent of conjugation of these aryl groups is discussed in more detail in section 3 . The simplest OAE wires are unfunctionalised OPEs (Fig. 1b), with purely hydrocarbon backbones. One of the simplest modifications of an OPE to afford an OAE is the replacement of one or more benzene rings with pyridine rings. In cases where pyridine rings are terminal, they typically act as anchor groups; such systems are discussed in the section 2.2.2. Pyridine func-

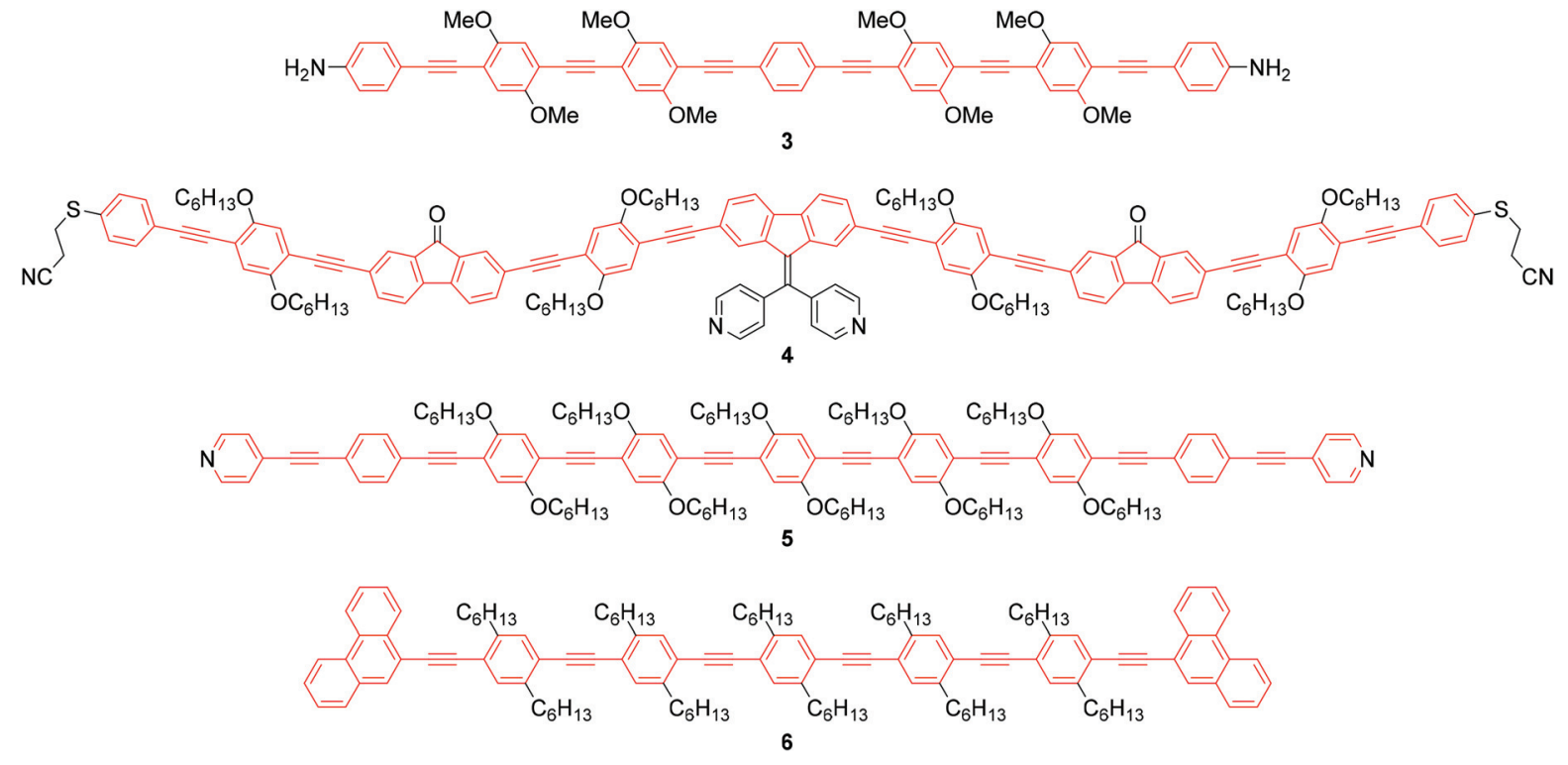

Fig. 9 Structures of selected long OAEs that have been studied in molecular junctions. The OAE backbones are highlighted in red. The end-groups shown served as the anchors, except for 4 which was deprotected in situ to give the thiolate. 

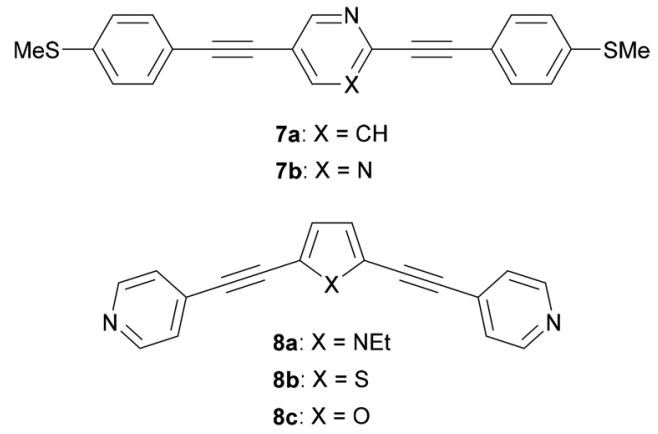

Fig. 10 Structures of some OAEs with simple heterocycles in the conductive backbone.

tionality can also be included elsewhere in the OAE backbone, e.g. as the central ring of an OAE3 in 7a, Fig. $10 .^{131}$ QI effects have been studied by varying the location of the nitrogen atom of a central, meta-disubstituted pyridine in a series of OAE3s, ${ }^{132}$ see section 3.4.

Other simple heterocycles are also readily incorporated into OAEs as either terminal or proximal aryl units. In some cases, terminal heterocycles can be used directly as anchoring units (see section 2.2.2). Examples of heterocycles which have been used as proximal aryl units include pyrrole, thiophene, furan and selenophene (e.g. 8a-c, Fig. 10). ${ }^{118,131,133,134}$ Note that the inclusion of 5-membered heterocycles results in more angular molecular wires compared to linear para-linked OPEs. Incorporation of heterocycles can have undesired effects: $7 \mathbf{b}$ (Fig. 10), an OAE3 with a central pyrimidine ring, was observed to display two distinct conductance features in STM-BJ experiments. One was assigned to conventional terminal anchoring, the second was attributed to additional anchoring interactions with the pyrimidine ring. ${ }^{131}$

Polycyclic systems have been widely studied, particularly as the central units of symmetric OAEs. Purely hydrocarbon units include biphenyl (9), ${ }^{68,135-137}$ fluorene (10), ${ }^{68,135-138}$ naphthalene (11), ${ }^{125,136}$ anthracene (12), ${ }^{125,136,139}$ anthanthrene $(\mathbf{1 3})^{117,140}$ and azulene (14); ${ }^{141}$ example structures are shown in Fig. 11. Heterocyclic derivatives include carbazole (15a), ${ }^{137}$ dibenzothiophene (15b), ${ }^{137}$ dibenzofuran (15c) ${ }^{137}$ benzodifuran (16), ${ }^{142,143}$ benzodithiophene (17), ${ }^{66}$ bithiophene (18), ${ }^{68}$ and (metallo-)porphyrins (19); ${ }^{144-146}$ example structures are shown in Fig. 12.

OAEs containing ligating heterocyclic moieties such as bipyridine (20), ${ }^{147}$ phenanthroline $(\mathbf{2 1})^{148}$ and terpyridine $(22)^{149}$ have been used to investigate the effects of metal complexation on molecular conductance (Fig. 13). The conductance of 20 was shown to increase upon coordination to Pd. ${ }^{147}$ Switching of $\mathbf{2 1}$ upon Cu-complexation was anchor group dependent, ${ }^{148}$ as discussed in section 3.6. An OAE with a central terpyridine unit formed the iron complex 22, which exhibited bistable junction properties dependent on an applied electric field. This behaviour was attributed to a change in the spin state of the iron ion, from low- to highspin. This spin crossover is caused by distortion of the octahedral coordination sphere of $\mathrm{Fe}^{2+}$ upon alignment of the
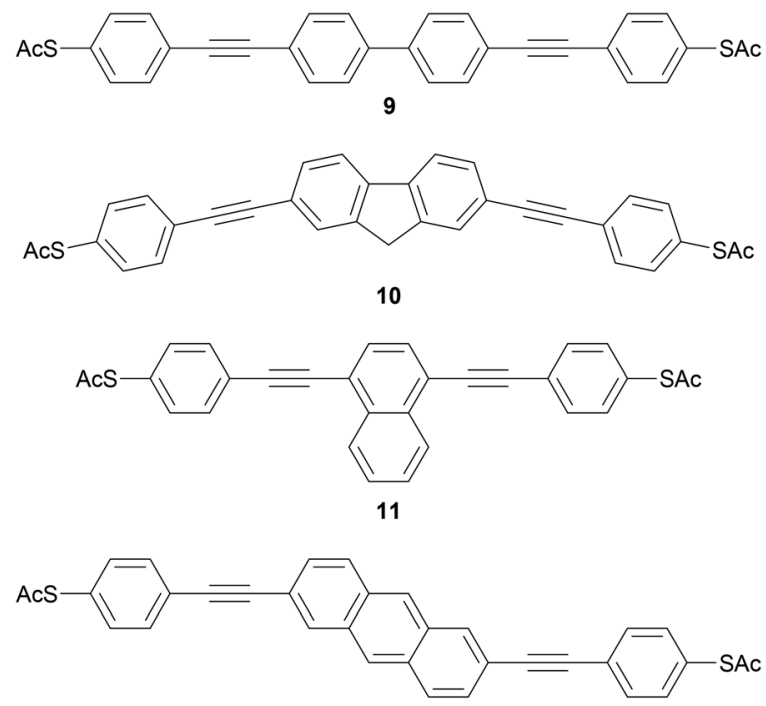

12

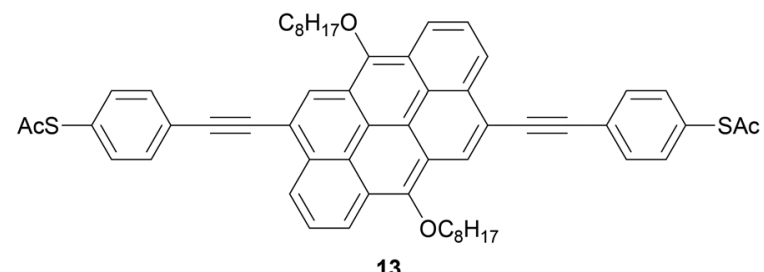

13

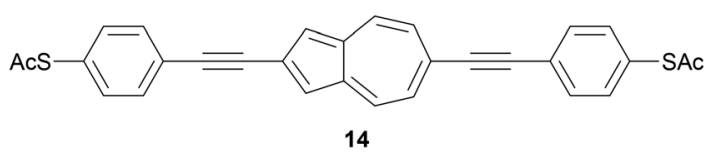

Fig. 11 Examples of OAE3 wires with a central polycyclic hydrocarbon.

dipole of the asymmetric second ligand with the applied field above a critical voltage. ${ }^{149}$ Notably, the use of an OAE architecture with isopropyl shielding groups significantly increased the proportion of junctions which displayed bistability compared to an unshielded oligoarylene analogue. This was related to a reduced potential for interaction between the functional subunit and the electrodes brought about by the increased distance from the electrodes and steric shielding. ${ }^{149}$

The molecular geometry of an OAE provides another source of structural variation. In the simplest example, difunctionalised benzene (or other 6-membered) rings can have ortho-, meta- or para-substitution. Early studies suggested that the single-molecule conductance of $o$-benzenedithiol (and $o$-phenylenediamine) could not be measured, and that the two anchor groups preferentially bind to the same electrode due to their close proximity. ${ }^{150}$ Subsequent developments in breakjunction technology have allowed the conductance of $o$-benzenedithiol to be measured, albeit with low junction formation probability $(<10 \%) .^{151}$ While for benzenedithiol isomers the length of the conductance pathway increases such that ortho $<$ meta < para, the conductance is predicted to follow the trend ortho > para > meta due to QI effects (see section 3.2), which was indeed observed experimentally. ${ }^{151}$ 


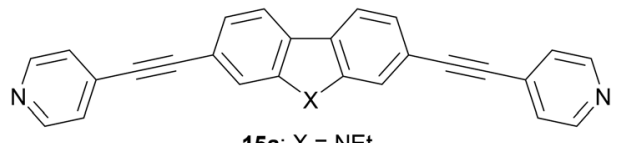

15a: $X=N E t$

15b: $X=S$

15c: $X=0$

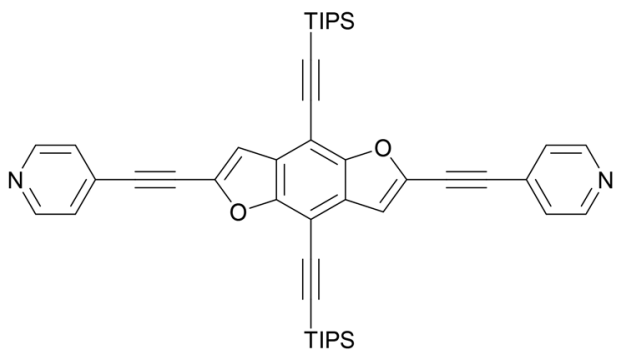

16

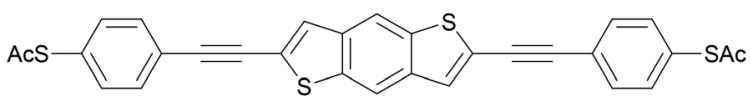

17

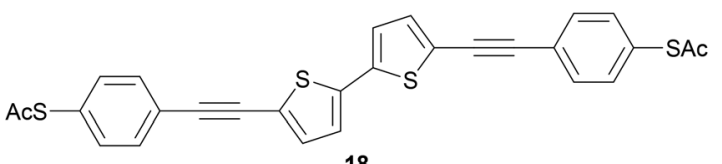

18

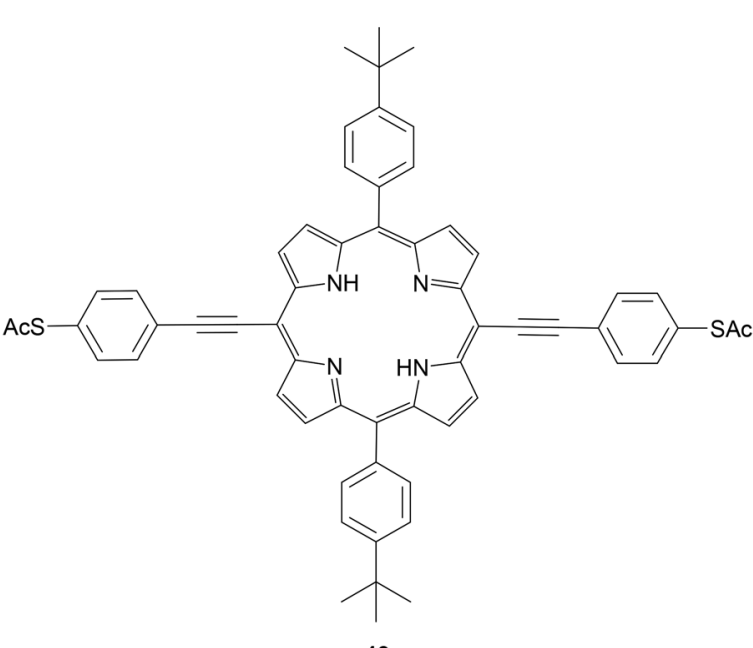

19

Fig. 12 Examples of OAE3 wires with a central polycyclic heterocycle.

Fig. 14 illustrates the same $o / m / p$-isomerism at the central ring of generic OPE3s (o-, $\boldsymbol{m}$ - and $\boldsymbol{p}$-OPE3). Like $\boldsymbol{o}$-benzenedithiol, the anchoring groups of $\boldsymbol{o}$-OPE3 are positioned such that they can more easily bind to the same electrode than to two different electrodes. This straightforward structural observation means that despite favourable QI properties, ortholinked OAEs are not prevalent in molecular junction studies. The rigid nature of alkyne bonds means that $\boldsymbol{m}$-OPE3 and $\boldsymbol{p}$ OPE3 can both easily bridge between two electrodes, and indeed many such species have been investigated in molecular junctions. $^{132,152,153}$
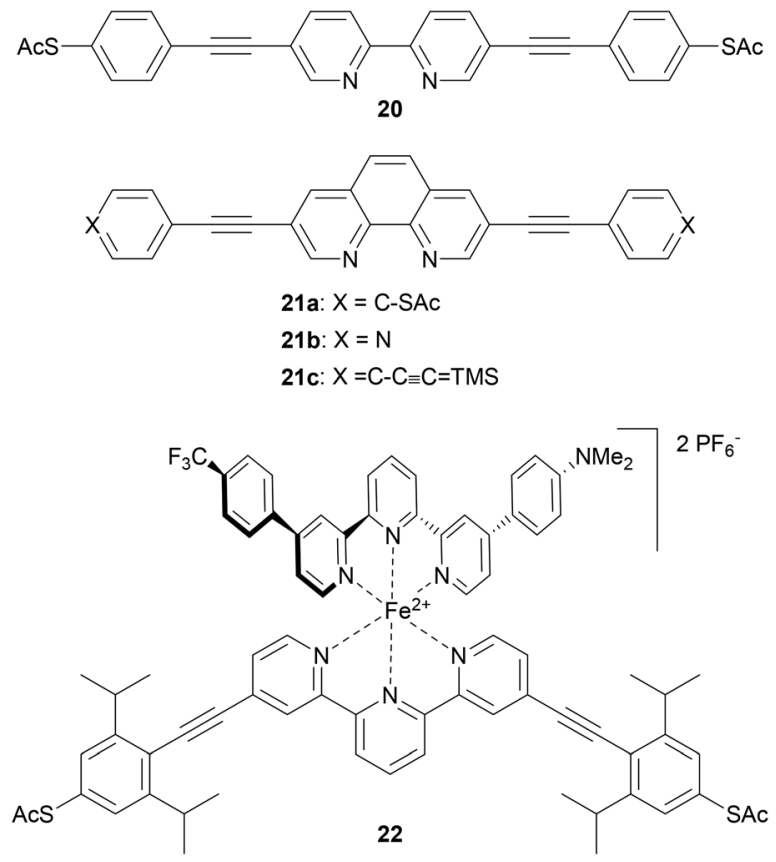

Fig. 13 OAE3 wires containing central units which can be used as ligands to form metal complexes.

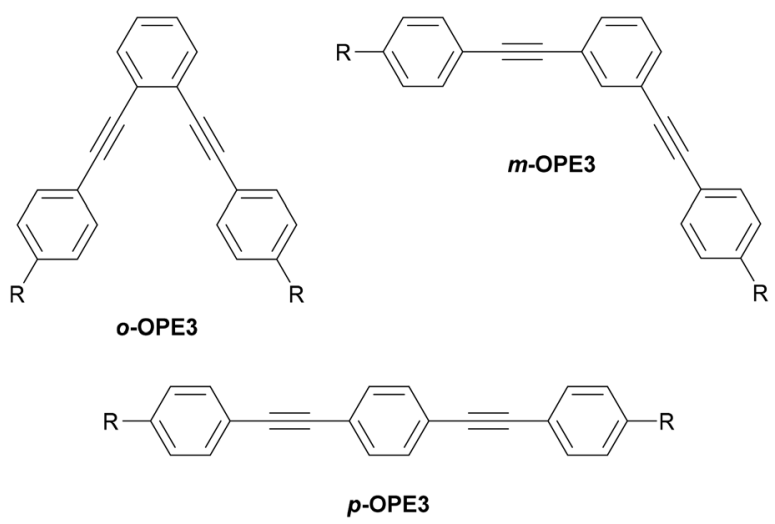

Fig. 14 Structural isomers of a generic OPE3 differing in their substitution pattern on the central ring. $\mathrm{R}$ denotes an anchoring group.

The location of the anchoring groups can be varied in a similar manner. The importance of this effect was shown experimentally in 2003 by comparing the OAE3 wires $\boldsymbol{m}$-23 and $\boldsymbol{p}$-23 (Fig. 15), which differ only in the connectivity of their anchoring groups relative to the OAE. ${ }^{154} p-23$ was found to be significantly more conductive than $\boldsymbol{m - 2 3}$.

More recently, Manrique et al. investigated a series of OAE3s with ortho-, meta- or para-pyridyl anchoring groups (relative to the backbone alkynes) and either para- or meta-connectivity at the central ring $(\boldsymbol{x}, \boldsymbol{y}, \boldsymbol{z}-\mathbf{2 4}$ in Fig. 15, where the preceding letters $x, y$ and $z$ indicate $o$-, $m$ - or $p$-geometry in the order anchor group, central ring, anchor group, i.e. left-toright as drawn). ${ }^{155}$ Their results also showed that para-connected anchoring groups afforded higher conductances than 

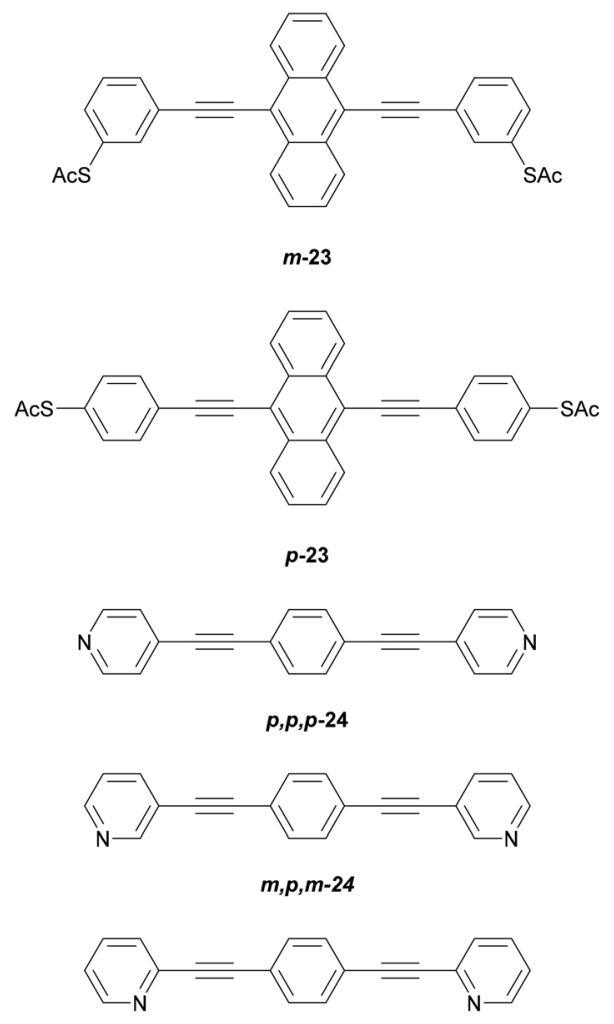

$0, p, 0-24$
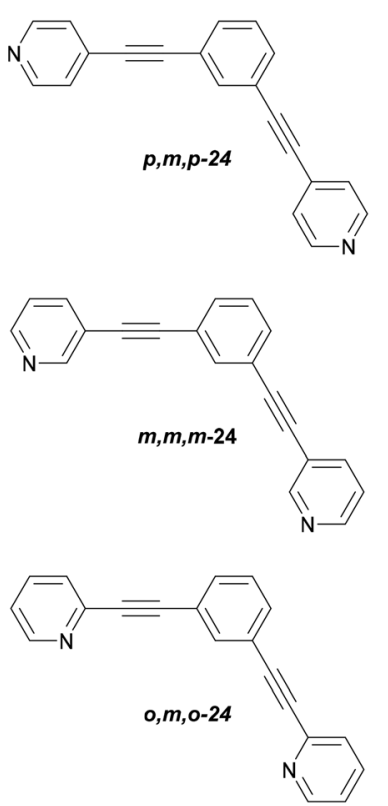

Fig. 15 OAE3 wires used to investigate the influence of anchoring group geometry.

their meta-isomers. Similarly to benzenedithiol isomers, orthoconnected anchoring groups were found to have low junction formation probability (attributed to steric effects), but higher conductance than their meta-isomers; indeed $p, p, p-24$ and $\boldsymbol{o , p}$, $\boldsymbol{o - 2 4}$ had the same conductance in STM-BJ measurements, an order of magnitude higher than $\boldsymbol{m}, \boldsymbol{p}, \boldsymbol{m}-\mathbf{2 4} .^{155}$ A further dis- advantage of ortho-anchoring was seen in the case of $\boldsymbol{o , m , o - 2 4}$, for which no conductance feature was observed. This was attributed to a combination of the short distance between the anchoring nitrogen atoms and a low expected conductance, which meant that any molecular conductance features were overlaid by the direct $\mathrm{Au}-\mathrm{Au}$ tunnelling current. ${ }^{155}$ The study also showed that para-connectivity at the central ring affords higher conductance than meta-connectivity; ${ }^{155} \boldsymbol{p}, \boldsymbol{p}, \boldsymbol{p}-\mathbf{2 4}, \mathbf{m}, \boldsymbol{p}$, $\boldsymbol{m - 2 4}$ and $\boldsymbol{o , p}, \boldsymbol{o}-\mathbf{2 4}$ were all at least an order of magnitude more conductive than their respective analogues, $\boldsymbol{p}, \boldsymbol{m}, \boldsymbol{p}-\mathbf{2 4}, \boldsymbol{m}, \boldsymbol{m}, \boldsymbol{m}$ 24 and $\boldsymbol{o}, \boldsymbol{m}, \boldsymbol{o}-\mathbf{2 4}$. Similar results have been consistently observed for OAE3 species with a range of anchoring groups. ${ }^{132,152,156,157}$ While para-connectivity is clearly advantageous in terms of electronic conductance, meta-linked species have favourable thermal properties. ${ }^{152}$ This will be discussed in more detail in section 4.2.

Geometric variation is not limited to ortho-, meta- and paraisomers of 6-membered rings. Many of the heterocyclic and polycyclic aryl units discussed above (e.g. Fig. 11 and 12) have been investigated in connectivity comparisons. ${ }^{117,118,125,126,137,138}$ However, it should be noted that as the complexity of the aryl unit increases it becomes less likely that all possible isomers can be easily synthesised, or indeed synthesised at all. The effects of varying connectivity in polycyclic and heterocyclic systems are discussed in detail in sections 3.2 and 3.4, respectively.

2.2.2 Anchoring groups. Table 1 shows a selection of anchoring groups that have been used to form OAE molecular junctions, inclusive of the terminal aromatic ring of the OAE unit. The following section discusses these and other anchors. Anchoring groups that are yet to be used in OAE-type molecules for junction studies are not considered in this review; the interested reader is directed to more general reviews of molecular electronics. ${ }^{46,158}$ OAE wires frequently have an anchoring group at each end, although species with a single anchor can still form junctions in break junction experiments, e.g. as $\pi$-stacked dimers ${ }^{159}$ or through $\pi$-gold interactions. ${ }^{123}$ For ensemble junctions the nature of the top contact means that an anchoring group is not necessarily required at this interface. All of the anchoring groups in Table 1 are shown in the para-position relative to the OAE backbone; as discussed in section 2.2.1 this is the preferred position because of steric availability and QI effects (see section 3.2). ${ }^{154,155}$

The most widely used anchoring groups for metal-molecule-metal (generally $\mathrm{Au}-$ molecule-Au) junctions are thiol $(-\mathrm{SH})$, pyridine $\left(-\mathrm{C}_{5} \mathrm{H}_{4} \mathrm{~N}\right)$ and amine $\left(-\mathrm{NH}_{2}\right)$ groups. ${ }^{160,161}$ These functionalities are usually easy to incorporate into an OAE structure and they afford sufficiently stable and reproducible junctions for conductance studies. ${ }^{161}$ While pyridine- and amine-bearing species can be assembled directly on surfaces, thiol assemblies are usually prepared from a protected precursor. This is because free thiols readily oxidise to form disulfides, which do not assemble efficiently and can result in polymerisation of wires bearing two thiol anchors. ${ }^{39}$ Thioacetates are archetypal masked thiols, as they can be deprotected under mild conditions to form thiolates. ${ }^{39,124,187}$ Notably, 
Table 1 Selected anchoring groups used in the formation of OAE molecular junctions, their anchoring mode to a relevant electrode and examples of their use. R represents the remainder of the OAE structure, beginning with an alkyne and inclusive of the remainder of the backbone, any side groups and additional anchoring groups where present. For simplicity, anchoring groups are shown attached to a benzene ring unless they already form part of the terminal aromatic system (e.g. pyridine derivatives). Note that anchoring groups can also be attached to other terminal aromatic species

Structure and name (abbreviation)<smiles>[R]c1ccc(S)cc1</smiles>

$\mathrm{R}$<smiles>[R]c1ccc(SC(C)=O)cc1</smiles>

Thioacetate (-SAC)

(Protected thiol)<smiles>[R]c1ccncc1</smiles>

Pyridine $\left(-\mathrm{C}_{5} \mathrm{H}_{4} \mathrm{~N}\right)$<smiles>[R]c1ccc(N)cc1</smiles>

Amine $\left(-\mathrm{NH}_{2}\right)$<smiles>[R]c1ccc(SC)cc1</smiles>

Methyl thioether / Methylthio (-SMe)<smiles>[R]c1ccc2c(c1)CCS2</smiles>

Dihydrobenzothiophene (DHBT)

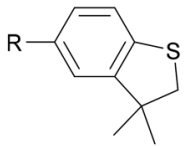

3,3-Dimethyldihydrobenzothiophene (DMBT)<smiles>[R]c1ccsc1</smiles>

3-Thienyl $\left(-\mathrm{C}_{4} \mathrm{H}_{3} \mathrm{~S}\right)$<smiles>[R]c1ccc([Se]C)cc1</smiles>

Methyl selenoether / Methylseleno ($\mathrm{SeMe})$<smiles>[R]c1ccc(C#N)cc1</smiles>

Cyano / Nitrile (-CN)<smiles>[R]c1ccc([N+](=O)[O-])cc1</smiles>

Nitro $\left(-\mathrm{NO}_{2}\right)$<smiles>[R]c1ccc([N+]#[C-])cc1</smiles>

Isocyano / Isonitrile (-NC)
Anchoring mode to typical surface

Covalent (S-Au), loss of $\mathrm{H}^{+}$

Covalent (S-Au), loss of $\mathrm{Ac}^{+}$

Dative $(\mathrm{N} \rightarrow \mathrm{Au})^{a}$

Dative $(\mathrm{N} \rightarrow \mathrm{Au})$

Dative $(\mathrm{S} \rightarrow \mathrm{Au})$

161,164

Dative $(\mathrm{S} \rightarrow \mathrm{Au})$

102, 157, 162 and 164

Dative $(\mathrm{S} \rightarrow \mathrm{Au})$

165 and 166
156 and 167

164

Dative $(\mathrm{Se} \rightarrow \mathrm{Au})$

160, 162 and 163
Literature examples (Ref. no.)

15 and 48

$160-163$

160-162

$160-162$ 
Table 1 (Contd.)

Structure and name (abbreviation)

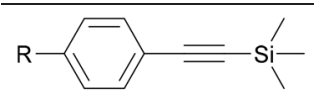

Trimethylsilylethynyl (-TMSE)<smiles>[R]c1ccc(C#C[Si]([R])([R])[R])cc1</smiles>

Trialkylsilyl-protected alkyne $\left(-\mathrm{C} \equiv \mathrm{C}-\mathrm{SiR}_{3}{ }_{3}\right)$,

$$
\mathrm{R}^{\prime}=\mathrm{Me},{ }^{\mathrm{i}} \mathrm{Pr}
$$

(Direct C-Au bond)<smiles>[R]c1ccc(C#C)cc1</smiles>

Alkyne $(-\mathrm{C} \equiv \mathrm{CH})$

(inert atmosphere)

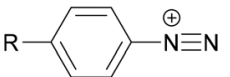

Diazonium as a precursor for electrochemical direct attachment $\left(-\mathrm{N}_{2}{ }^{+}\right)$ (Direct Ar-Au bond)

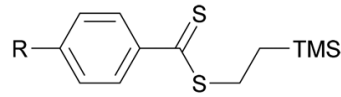

Trimethylsilylethyl-protected carbodithioate ( $-\mathrm{CS}_{2} \mathrm{CH}_{2} \mathrm{CH}_{2} \mathrm{TMS}$ )<smiles>[R]c1cn[nH]c1</smiles>

Pyrazol-4-y<smiles>[R]c1ccc(-c2nnco2)cc1</smiles>

1,3,4-Oxadiazol-2-yl

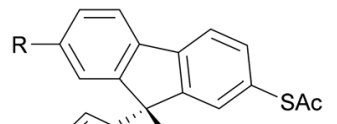<smiles>C=CC[C@@]1(C)c2ccc([As])cc2-c2cc(S(C)(C)C)ccc21</smiles>

Spirobifluorene tripod<smiles>[R]c1ccc(C(c2ccc(-c3ccncc3)cc2)(c2ccc(-c3ccncc3)cc2)c2ccc(-c3ccncc3)cc2)cc1</smiles>

Tetraphenylmethane-based tripod
Anchoring mode to typical surface

$\mathrm{e}^{-}$donation from $\mathrm{Au}(\mathrm{Au} \rightarrow \mathrm{Si})$

Covalent (C-Au), in situ removal of $\mathrm{SiR}_{3}^{\prime}$

Covalent (C-Au), loss of $\mathrm{H}^{+}$

Covalent (Ar-Au), loss of $\mathrm{N}_{2}$

Covalent ( $\mathrm{S}-\mathrm{Au})$, mono- or bidentate, in situ removal of TMS- $\mathrm{CH}_{2} \mathrm{CH}_{2}{ }^{+}$

Dative $(\mathrm{N} \rightarrow \mathrm{Au})$

Covalent $(\mathrm{S}-\mathrm{Au} \times 3)$

$\pi$-Interaction $(\pi-\mathrm{Au})$
182
Literature examples (Ref. no.)

170 and 171

142, 172 and 173

174 and 175

122

143, 145 and 176

179-181 
Table 1 (Contd.)

Structure and name (abbreviation)

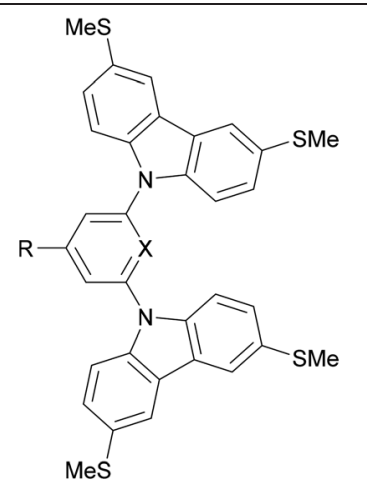

Carbazole-based tetrapod, $\mathrm{X}=\mathrm{CH}, \mathrm{N}$

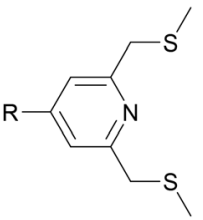

Bis((methylthio)methyl)pyridine

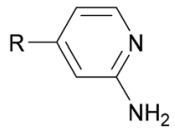

2-Aminopyridine

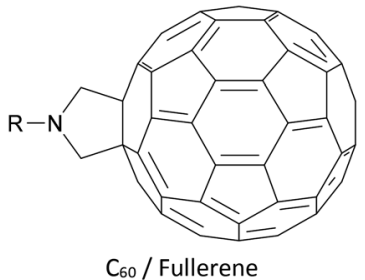

(One backbone bonding strategy shown)

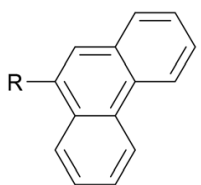

Phenanthrene

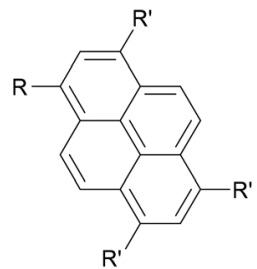

Pyrene $\left(\mathrm{R}^{\prime}=\mathrm{H}\right)$

1,3,8-Tridodecyloxypyrene $\left(\mathrm{R}^{\prime}=\mathrm{OC}_{12} \mathrm{H}_{25}\right)$
Anchoring mode to typical surface

Dative $(\mathrm{S} \rightarrow \mathrm{Au} \times 4)$ and $\pi$-Au

Dative $(\mathrm{N} \rightarrow \mathrm{Au}$ and $\mathrm{S} \rightarrow \mathrm{Au} \times 2)$

Dative $(\mathrm{N} \rightarrow \mathrm{Au}$ and $\mathrm{N} \rightarrow \mathrm{Au})$

$\pi$-Interaction $(\pi-\mathrm{Au})$

$\pi$-Interaction $\left(\pi-\pi_{\mathrm{CNT}}\right)$

$\pi$-Interaction $\left(\pi-\pi_{\mathrm{Graphene}}\right)$
183

186

76 and 77

Literature examples (Ref. no.)

60

184 and 185

$73-75$ 
Table 1 (Contd.)

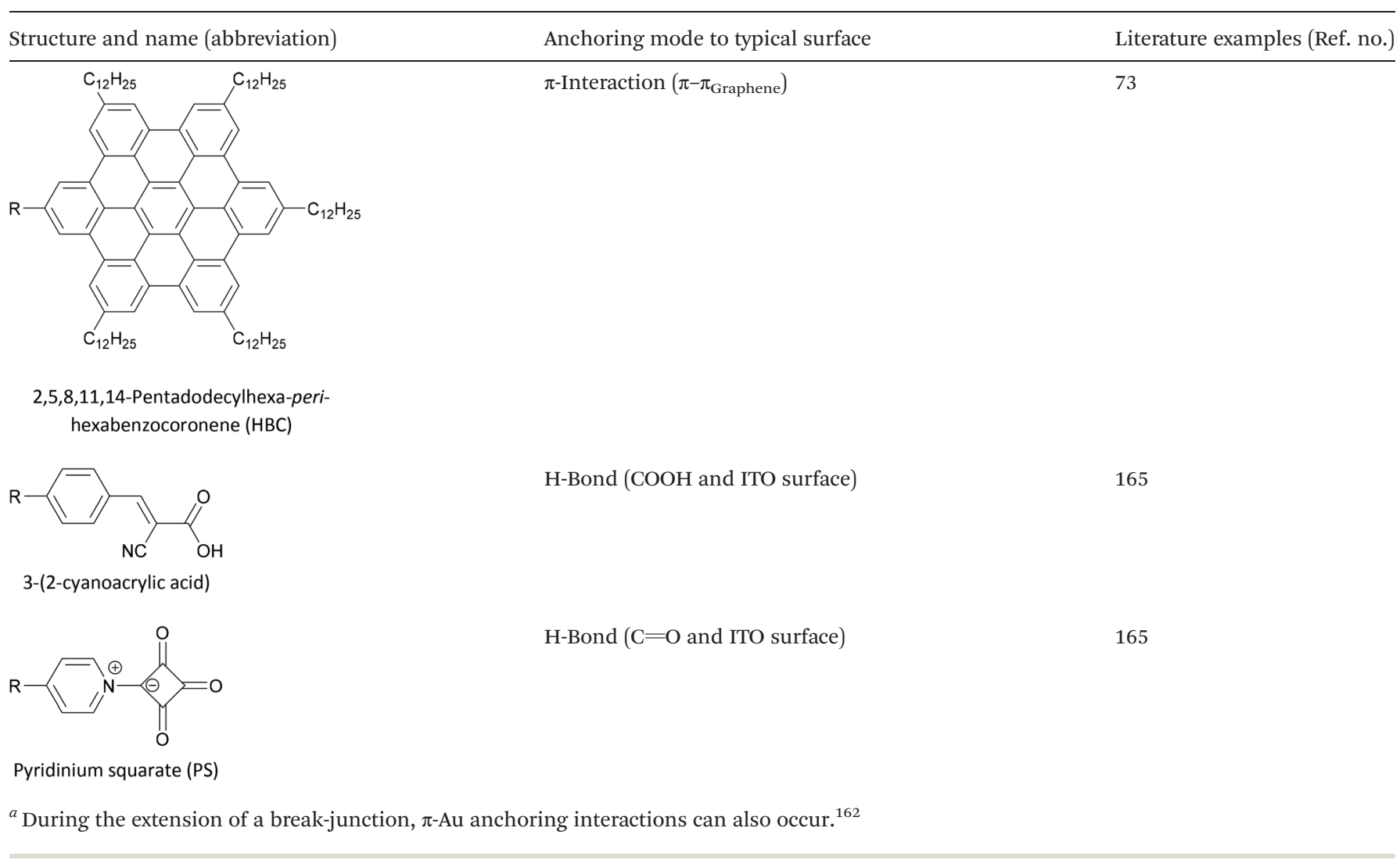

thioacetates spontaneously cleave in the presence of gold to form the direct $\mathrm{Au}-\mathrm{S}$ bonds that would be expected from thiols or thiolates. ${ }^{39}$ Investigations comparing assemblies prepared via spontaneous cleavage to those formed using a deprotecting agent suggest that the optimal method depends on the proposed application. ${ }^{124,187}$ For experiments where a densely packed SAM is not required, such as single-molecule conductance studies, chemical deprotection is not necessary. However, controlled use of a suitable basic deprotecting agent can facilitate the formation of densely packed high-quality SAMs suited to ensemble studies. ${ }^{124}$ Cyanoethyl ${ }^{129}$ and trimethylsilylethyl ${ }^{188}$ protecting groups have also been used to mask thiols, although in these cases addition of deprotecting agents is necessary in order to form junctions.

Thioether-based anchors, such as methyl thioether (methylthio, -SMe $)^{161,164}$ and dihydrobenzothiophene $(\mathrm{DHBT})^{102,157}$ derivatives have seen increasing use in recent years as they allow for strong Au-S interactions (albeit weaker than those between thiols and gold ${ }^{161}$ ), can assemble directly on surfaces, and have wider chemical compatibility during synthesis than protected thiols. An important difference between -SMe and -SAc anchoring groups is that the methyl group is retained upon binding to a gold surface whereas the acetate group is not; the lower conductance of thioethers relative to thiols is attributed to this phenomenon. ${ }^{161}$ The dimethylated analogue of DHBT, namely DMBT, is also an effective anchor for gold surfaces. ${ }^{165,166}$ Thiophene has been used as a sulfur-based anchor, usually as a 3-thienyl $\left(-\mathrm{C}_{4} \mathrm{H}_{3} \mathrm{~S}\right)$ substituent. ${ }^{156,167}$ Selenium-based anchoring groups, for example methyl selenoether (methylseleno, -SeMe), have been shown to have similar properties to their sulfur analogues. ${ }^{164}$ Their lack of widespread use is therefore not surprising, given the lower availability and increased cost of precursor molecules compared to sulfur species and the foul smell of many organoselenium compounds.

Other simple functional groups which have been utilised for anchoring include cyano- (nitrile, $-\mathrm{CN}$ ), ${ }^{160,163}$ nitro$\left(-\mathrm{NO}_{2}\right)^{163}$ and isocyano- (isonitrile, $\left.-\mathrm{NC}\right)^{168}$ moieties. These do not anchor as effectively or reliably as the species discussed above, and so are less commonly used. For example, isocyanoanchored OPEs were observed to desorb from gold surfaces upon exposure to air through decomposition to isocyanates. ${ }^{169}$ Carboxylic acid functionalised OPEs have been assembled in Langmuir-Blodgett (LB) films, transferred onto gold surfaces and captured in molecular junctions using an STM. ${ }^{189}$ Recent results show that a pyridinium ring can be an effective anchor in the presence of an external electric field. ${ }^{190}$

Trialkylsilylethynyl groups are often present in intermediates used in the preparation of OAEs (see section 2.1). They have also been applied to anchoring in two different ways. Trimethylsilylethynyl (TMSE) groups can bind with a gold surface through the silicon atom. This is attributed to a switch from a tetrahedral to a trigonal bipyramidal geometry upon electron donation from the gold surface. ${ }^{170}$ The resulting molecular junctions give well-defined conductance features, attributed to 
the steric bulk of the TMS group, although conductance is lower than for common anchoring groups. ${ }^{170,171}$ In an alternative approach, in situ treatment of TMSE ${ }^{172}$ or triisopropylsilylethynyl (TIPSE) $^{142,173}$ terminated OAEs with tetrabutylammonium fluoride (TBAF) allows for assembly of molecular wires on metallic surfaces through direct alkyne-metal bonds, i.e. $\mathrm{R}-\mathrm{C} \equiv \mathrm{C}-\mathrm{Au}$. The single-molecule conductance of such junctions was shown to be higher than that of comparable thiol-anchored species. ${ }^{172}$ However, within $1 \mathrm{~h}$ surface-mediated oxidative dimerization of the deprotected species results in the formation of oligomers, which can also be captured in junctions, complicating data analysis. ${ }^{172}$ Direct alkyne binding has been applied to functionalisation of $\mathrm{Au}, \mathrm{Pt}, \mathrm{Pd}, \mathrm{Rh}, \mathrm{Ir}$ and highly ordered pyrolytic graphite (HOPG) surfaces, although rehybridisation of the atoms involved in bonding may occur in some cases. ${ }^{191}$ Switching between pyridyl and alkyne anchoring during the course of a MCBJ experiment has been achieved using 16 (Fig. 12) though in situ deprotection of the TIPSE units. ${ }^{142}$

Assembly of alkynes on gold has also been described using hydrogen-terminated alkynes as the anchoring group precursor, with formation of $\mathrm{R}-\mathrm{C} \equiv \mathrm{C}-\mathrm{Au}$ linkages. ${ }^{174,175}$ The reported assembly conditions usually specify an inert atmosphere to prevent oxidation of the alkyne functionality, and an overnight assembly period, indicating that this method is slower and more sensitive than those based on in situ deprotection. Aumolecule-Au junctions have additionally been formed from assemblies of OAE or OAE-like molecules bearing a terminal alkyne-Au complex. ${ }^{192,193}$ After self-assembly or LB film transfer onto a gold substrate (using a conventional anchoring group), thermally-induced decomposition of the Au complex results in the loss of ancillary ligands, a reduction from $\mathrm{Au}(\mathrm{I})$ to $\mathrm{Au}(0)$ and the formation of $\mathrm{Au}$ nanoparticles (NPs) on top of the molecular monolayer. The conductance of the resulting $\mathrm{Au}-[\text { molecule }]_{x^{-}}$ $\mathrm{Au}_{\mathrm{NP}}$ junctions, containing one or more molecular wires, can be investigated by contacting the NPs with a c-AFM tip. Another method to form a direct $\mathrm{C}-\mathrm{Au}$ bond relies on the use of aryldiazonium salts, which can be electrochemically grafted to gold surfaces with loss of $\mathrm{N}_{2}$, forming a direct $\mathrm{Ar}-\mathrm{Au}$ bond. ${ }^{122}$

The carbodithioate $\left(-\mathrm{CS}_{2}{ }^{-}\right)$moiety has been shown to anchor effectively to gold surfaces and afford an order of magnitude increase in single-molecule conductance versus equivalent thiols for several different conductive backbones. ${ }^{143,145,176}$ The molecules used in these studies are prepared as their 2-(trimethylsilyl)ethyl carbodithioate esters, which can be deprotected in situ with TBAF. The limited uptake of this promising anchoring group may relate to the poor availability of a key precursor material, (2-bromoethyl)trimethylsilane. Mono- and bidentate binding modes were observed in the same STM-BJ study of a symmetric OAE3 wire with carbodithioate anchors, with different conductance features assigned to junctions in which both, one, or neither of the anchoring groups bound in bidentate fashion. ${ }^{145}$ Dithiafulvene (DTF) derivatives have also been investigated as anchors with multiple sulfur contact points. They functioned more reliably in ensemble than in single-molecule junctions, but studies are limited and the precise nature of the anchoring remains unclear. ${ }^{194,195}$
Two distinct anchoring modes have also been observed when pyrazole is used as a terminal group. ${ }^{177}$ As a break junction containing a pyrazole-anchored OAE3 was stretched, the molecular conductance increased prior to breaking of the junction. This was attributed to a switch from a monodentate, dative interaction between the pyridine-like nitrogen and the STM tip to a bidentate covalent interaction following loss of the proton from the pyrrole-like nitrogen. ${ }^{177}$ Oxadiazole is another small heterocycle that has been applied as an anchoring unit, again with evidence to support different binding conformations. $^{178}$

To increase the stability of molecular assemblies for molecular junctions and other applications, multipodal anchoring units incorporating several anchoring groups have been designed. ${ }^{196}$ Additional binding functionality increases the strength of molecule-surface interactions relative to a single anchoring group. The designs used to achieve this effect often have a larger molecular footprint than simple anchor groups and so occupy a higher surface area per molecule, resulting in a less dense molecular assembly; this can be useful as a means of preventing intramolecular interactions. Multipodal anchoring units that have been used in OAE wires include a spirobifluorene-based tripod, ${ }^{179-181}$ a tetraphenylmethanebased tripod ${ }^{182}$ and carbazole-based tetrapods. ${ }^{60}$ Smaller-footprint approaches using a pyridyl anchor buttressed by two (methylthio)methyl substituents, ${ }^{183}$ and a bidentate 2-aminopyridine anchor ${ }^{184,185}$ have also been reported.

The synthesis of OAEs bearing $\mathrm{C}_{60}$ (fullerene) moieties in anchoring positions was reported in the early 2000s. ${ }^{197,198}$ Despite this, only simple disubstituted benzene ("OAE1") systems with $\mathrm{C}_{60}$ anchors have been used to form molecular junctions, ${ }^{186}$ although $\mathrm{C}_{60}$ anchors have been utilised in larger systems with other molecular backbones, ${ }^{199}$ and $\mathrm{C}_{60}$-terminated OPE hybrids have been prepared for D-B-A studies. ${ }^{80}$

Increasing interest in junctions based on graphitic materials has led to new anchoring approaches. The extended $\pi$-system of materials such as graphene and CNTs is well suited to large, planar aromatic anchor groups, which bind through $\pi-\pi$ interactions. These aromatic systems are readily incorporated as the terminal aryl groups of OAE-like wires. Phenanthrene-terminated OAEs and OAE-like systems such as 6 (Fig. 9) have been shown to bridge CNT nanogaps and form CNT-molecule-CNT junctions. ${ }^{76,77} 6$ has also been partially lifted from a Au(111) surface using an STM tip and the properties of the resulting Au-(sub-)molecule-tip junctions investigated. ${ }^{130}$ Graphene nanogaps have been bridged using porphyrin-based OAEs bearing (substituted) pyrene or alkylated hexa-peri-hexabenzocoronene (HBC) anchoring groups. ${ }^{73-75}$ Continued interest in the unique properties of graphene means that the development of additional specialised anchoring groups of this type can be expected. CNT nanogaps have also been covalently bridged by OAEs with amine anchors, which can react with carboxylic acid functionality at the CNT edge to form amides. ${ }^{200}$ A similar covalent approach based on in situ imine formation was used to bridge a silicon nanogap. ${ }^{72}$ 
Indium tin oxide (ITO) is a widely used electrode material in organic light emitting diodes and photovoltaics as it is both transparent and conductive. ITO-molecule-Au junctions containing asymmetric OAE2 species were recently investigated using the $I(t)^{51}$ modification of the STM-BJ method. ${ }^{165}$ Carboxylic acid, 2-cyanoacrylic acid, and pyridinium squarate (PS) were all shown to be suitable anchoring groups for ITO. PS showed no affinity for Au surfaces, meaning selective junction orientation could be achieved using a species bearing both SAc and PS anchor groups. ${ }^{165}$ Selectivity studies of this type are of relevance to controlled device manufacturing, and comparable studies with other electrode materials would be insightful.

2.2.3 Role of side groups. Side groups are commonly needed to increase the solubility of OAE wires, particularly longer systems. ${ }^{112,123,128}$ They can additionally facilitate synthesis, purification and conductance studies by increasing the range of suitable solvents and reducing the propensity of OAEs to stack through $\pi-\pi$ interactions, which can complicate single-molecule conductance data by providing additional signals relating to e.g. $\pi$-stacked dimers. ${ }^{123}$ Side groups also provide a means to incorporate further functionality into OAE wires.

Simple alkyl or alkoxy chains are generally sufficient for solubility enhancement. In an important early study, Huber et al. first showed that the addition of two methoxy or hexyloxy substituents (25b and 25c, respectively, Fig. 16) to the central benzene ring of unsubstituted OPE3 (25a) resulted in no significant change to conductance histograms or the most probable conductance obtained via MCBJ. ${ }^{93}$ The tert-butylated analogue (25d) was later shown to have comparable conductance to 25 b using the $I(s)$ method. ${ }^{123}$ Similar results were subsequently observed for pyridine-anchored analogues with and without methoxy subsituents, ${ }^{112}$ and for a series of amineanchored OPE3s bearing a wider range of substituents on the central ring (including alkyl, alkoxy and fluoride substituents). ${ }^{201}$ In the latter case some concentration effects were noted and attributed to differing intramolecular interactions. $^{201}$ It should be noted that these studies investigated only symmetrically substituted, para-linked OAEs. In contrast, the addition of methoxy side groups to different positions on a meta-linked OPE3 substantially impacts on the molecular conductance due to QI effects, ${ }^{153}$ as discussed in section 3.4. Computational studies have demonstrated this effect for a

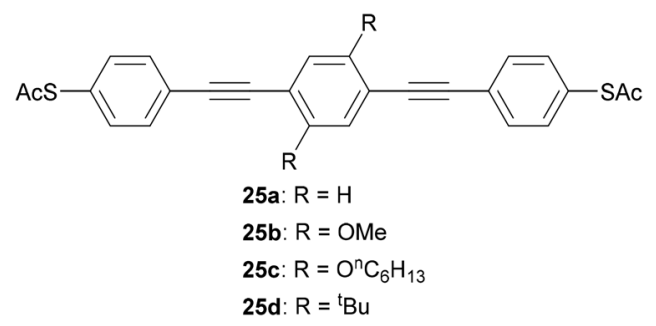

Fig. 16 Examples of OPE3 species used to show that side groups have little effect on molecular conductance for para-connected systems. range of electron-withdrawing and electron-donating side groups. $^{35,36}$ Side group functionalisation with alkoxy chains and halides has also been found to affect thermoelectric properties, ${ }^{202,203}$ as discussed in section 4.2.

Nitro side groups were associated with negative differential resistance (NDR) in early studies of molecular junctions. ${ }^{204}$ NDR was studied in SAMs of thiol-terminated nitro-functionalised OPE3 molecules. ${ }^{205}$ It was concluded that under certain assembly conditions extensive redox reactions can occur involving oxidation of the sulfur with concomitant reduction of nitro to amine, thereby impacting the reproducibility and the analysis of the electrical properties of the assemblies. ${ }^{205}$ It is notable that nitro groups are rarely seen in more recent experimental conductance studies.

While linear alkyl chains suffice to enhance solubility, other approaches provide more extensive shielding of OAE backbones from the surrounding environment. Wang et al. studied the series of dendron-functionalised OPE3s shown in Fig. 17 in various $\mathrm{Au}-$ molecule-Au junctions. ${ }^{206}$ In ensemble junctions, increased dendron size was associated with lower conductance per area. This was in part accounted for by X-ray photoelectron spectroscopy (XPS) data, which showed a
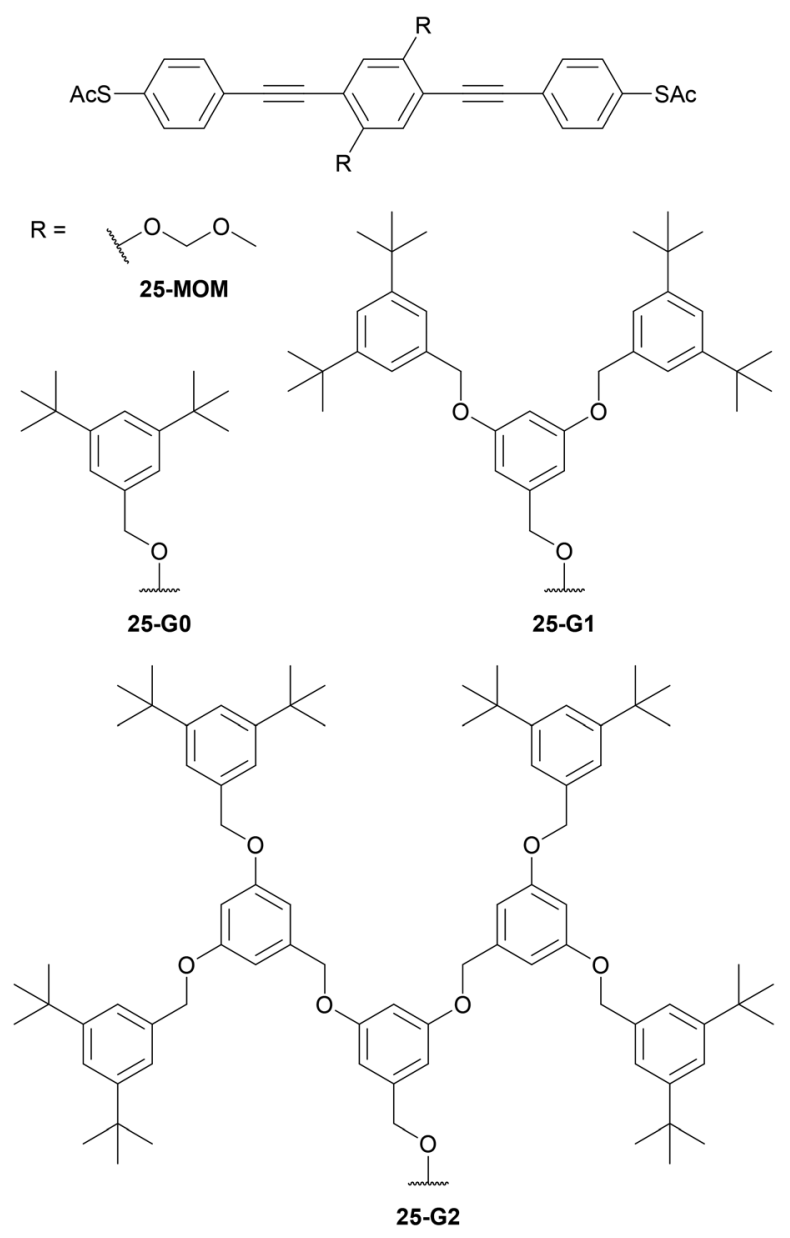

Fig. 17 Dendron-functionalised OPE3s used to investigate shielding of the molecular backbone. 
reduced SAM density with increasing side group size. Additional discrepancy within the series was ascribed to increased SAM disorder for the species with larger dendrons, which further reduced the number of molecules bridging the two electrodes. ${ }^{206}$ In STM-BJ experiments, reference compounds (monothiolated OPE3 and 25-MOM) afforded a conductance feature that was attributed to a $\pi$-stacked dimer. This was not observed for dendron functionalised species with bulky side groups. Conductance histograms for 25-MOM and 25-G0 contained features attributed to junctions comprising either one or two molecules, whereas that of bulkier 25-G1 displayed only the single-molecule feature. ${ }^{206}$ The implication is, therefore, that the smaller side chains are not sufficiently bulky to prevent the formation of multi-molecule junctions. The conductance histogram of 25-G2 was essentially featureless, suggesting that overly-large dendrons are not well-suited to molecular junction experiments, presumably as they can shield the anchoring functionality in addition to the backbone. ${ }^{206}$

Even more extensive shielding of an OAE backbone can be achieved by encapsulating it within a macrocycle, such as $\alpha$-cyclodextrin, to form a rotaxane. Extensive synthesis of long OAEs functionalised with multiple cyclodextrin units, including control of which positions are shielded, has been reported. ${ }^{207}$ Despite this, relatively few OAE rotaxanes have been the subject of conductance studies. ${ }^{208-210}$ The OAE backbones of rotaxane species 26 and 27 (Fig. 18) are respectively encircled by one or two $\alpha$-cyclodextrin macrocycles, which are attached to the OAE as side groups. In the case of 26, rotaxane shielding successfully prevented multiple molecules binding in STM-BJ experiments. It was also shown that junctions containing 26 displayed an asymmetric response to mechanical strain. Other than this, the authors state that the addition of the cyclodextrin ring had "no clear effect" on molecular conductance properties. ${ }^{208}$ In contrast, the shielding by the cyclodextrin rings in [3] rotaxane 27 was reported to afford sharp conductance features in STM-BJ experiments due to the restriction of intramolecular rotations, but features corresponding to multiple molecules in the junction were observed. ${ }^{210}$ Neither of these studies included a direct comparison with an unshielded analogue using the same STM-BJ equipment, making it difficult to confidently discern the overall effect of rotaxane shielding on molecular conductance.

DTF is an interesting functional side group that has been used by Nielsen and coworkers to produce a range of extended tetrathiafulvalene (exTTF) cruciform OPE molecular wires, ${ }^{56,194,195,211}$ exemplified by structures 28-30 in Fig. 19. (Species 30 is an example of an OPE with DTF-derived anchoring units.). exTTF cruciforms are of interest as candidate molecular redox switches. The OPE5 analogue of $\mathbf{2 8}$ has been investigated in a three-terminal device under gating and a magnetic field (see section 5.3 for further discussion). ${ }^{211}$ However, to our knowledge, studies of similar systems are limited to conductance measurements in the ground state. This may relate to poor reversibility in electrochemical studies. ${ }^{195}$ In c-AFM studies it was found that conductance increased as more DTF units were added to an OPE backbone. ${ }^{195}$ In contrast, in single-molecule studies (STM-BJ and MCBJ) little variation in conductance was seen for different numbers of DTF units. Furthermore, junction formation became less likely as the number of DTF units increased; this effect was attributed to the presence of increasing numbers of sulfur atoms, which could reduce the mobility of the molecular wires on gold surfaces. ${ }^{195}$ The differing trends between single-molecule and ensemble conductance measurements are believed to be due to both thioacetate protecting groups cleaving in the singlemolecule studies, but only that of the surface-bound sulfur in the c-AFM experiments. ${ }^{195}$

Other approaches have been used to add switching or sensing behaviour to OAE wires through side groups. Liu et al. investigated an OPE3 dithiol wire with dimesitylboryl side groups (25e, Fig. 20). ${ }^{212}$ The boron atoms can act as Lewis
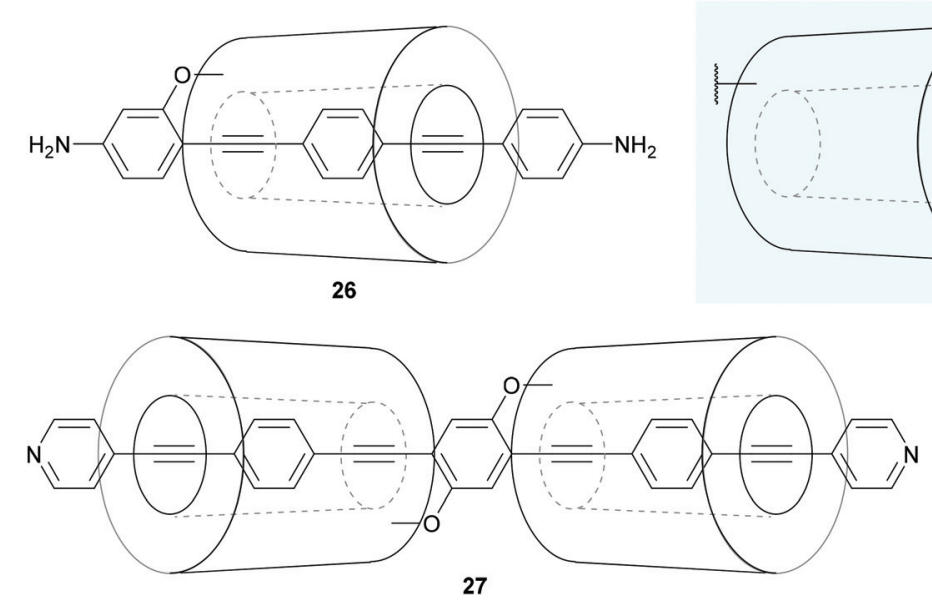

27

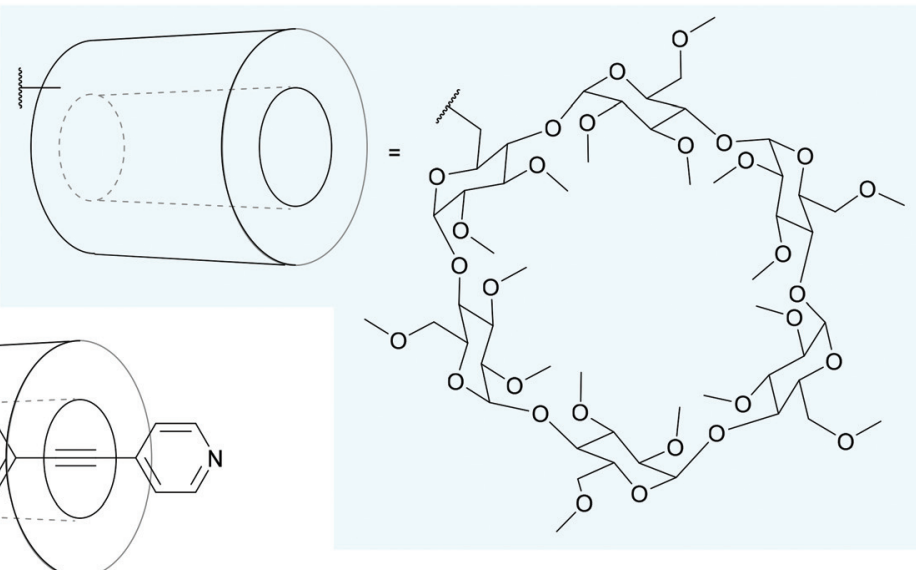

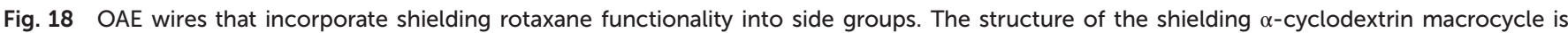
shown on a pale blue background. 


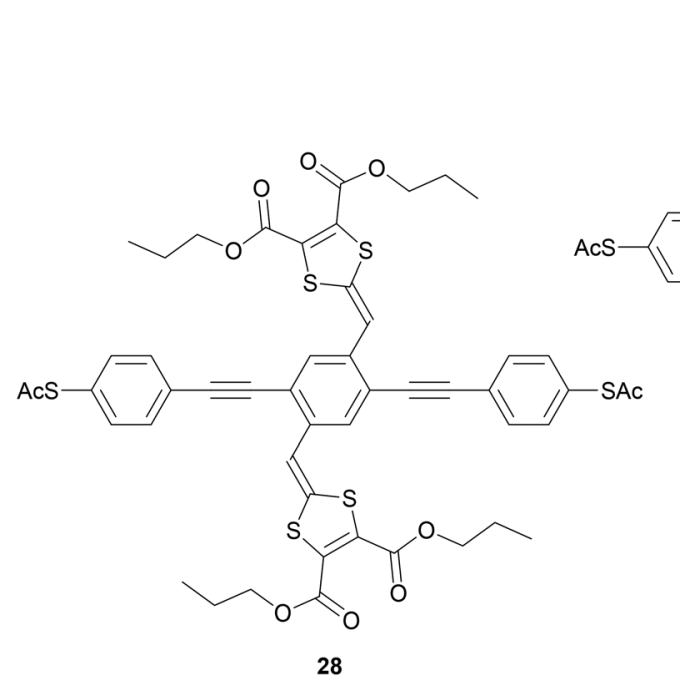

28

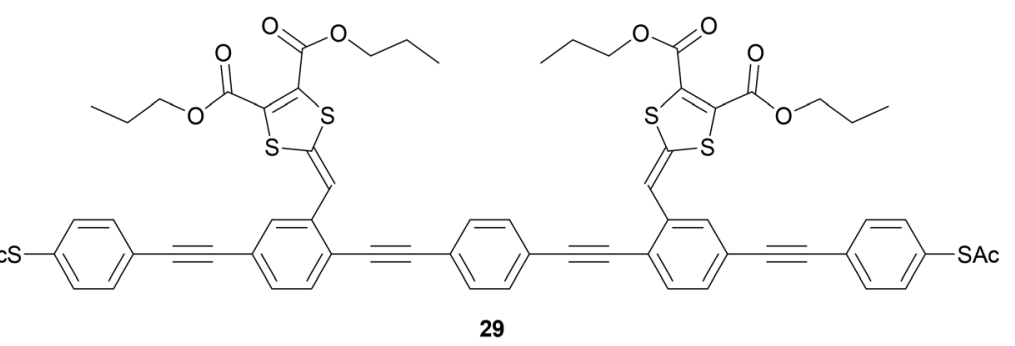

Fig. 19 Examples of DTF-functionalised exTTF OPE wires, ${ }^{195}$ with DTF units used as side chains on the same (28) or different (29) phenylene units, or as anchoring groups (30).

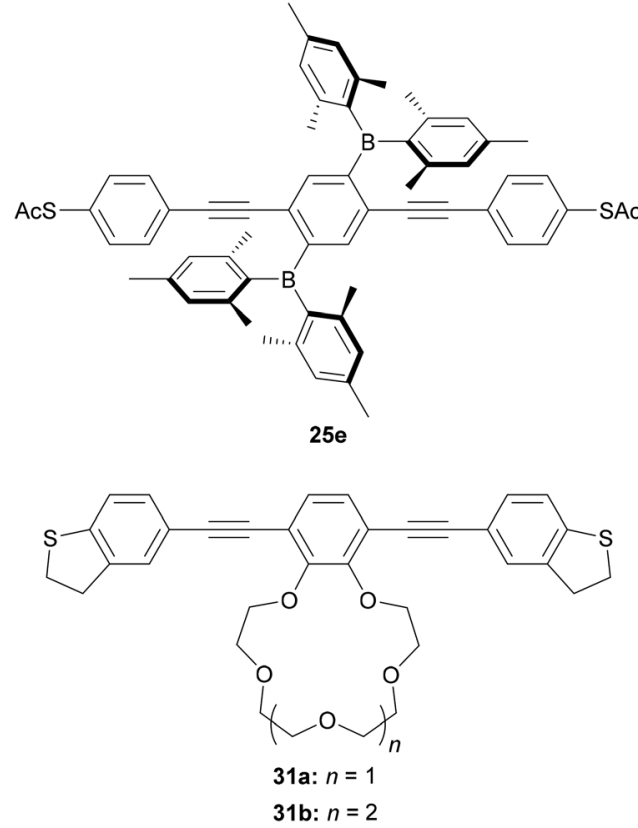

Fig. 20 Structures of OAE wires with side chain functionality that imparts switching properties through ion binding.

acids, which results in a $c a$. fourfold reduction in the conductance of 25e upon exposure to fluoride anions. The authors attribute this to a widening of the HOMO-LUMO gap upon fluoride binding, with support from charge transport simulations. $^{212}$ The reversibility of this switching was not investigated. Cation sensing is also possible. The conductance of a DHBT-anchored OPE3 with a 15-crown-5 type side group (31a, Fig. 20) increased by a factor of up to ca. 2 upon complexation with $\mathrm{Li}^{+}, \mathrm{Na}^{+}$or $\mathrm{Rb}^{+}$. The magnitude of the increase was shown to correlate with the ionic radius of the bound ion. ${ }^{213}$ A larger, $\mathrm{ca}$. four-fold increase was seen upon complexation of $\mathrm{K}^{+}$. This was attributed to the formation of a $2: 1$ 31a : $\mathrm{K}^{+}$complex in which both wires bridged between the electrodes of the STM-BJ based on charge transport simulations. ${ }^{213}$ Furthermore, STM-BJ studies of $\mathbf{3 1 b}$, with a larger 18-crown-6 type side group, for which 1:1 complexation of $\mathrm{K}^{+}$is expected, afforded a conductance in line with the trend observed for the other alkali metal cations. ${ }^{213}$ Again, the study did not consider the reversibility of this switching. The confirmation or development of reversible ion sensors is desirable if such materials are to be applied practically in devices.

2.2.4 OAEs linking other functionality. There are many examples of OAE derivatives that do not strictly fit the definition of OAEs as (hetero)aromatic ring systems linked by alkynes in an alternating fashion. Molecular wires with a central functional unit linked to anchor units by OAE-type backbones are common. Such species can provide useful references when compared to analogous OAEs, and have the potential to function as more complex circuit components, such as molecular switches. They are therefore highly relevant to this review.

Molecular wires containing cross-conjugated quinoidal units (Fig. 21), in particular anthraquinone derivatives such as 32, are well studied. They have been used in comparisons with linearly conjugated (anthracene, e.g. 12), and non-conjugated (dihydroanthracene) analogues. ${ }^{125,214}$ Comparisons have also been made with other cross-conjugated structures based on anthracene, including tetracyanoquinodimethane (TCNQ) derivatives (e.g. 33) and exTTFs (e.g. 34). ${ }^{67}$ These and anthraquinone wires can function as redox-switches by gaining aromaticity and becoming linearly conjugated as their oxidation state is changed, ${ }^{63,215-217}$ either electrochemically or by formation of charge-transfer salts. Quinoidal wires can also be 
<smiles>CC(C)(C)c1ccc(C#Cc2ccc3c(c2)C(=O)c2ccc(C#Cc4ccc(C(C)(C)C)cc4)cc2C3=O)cc1</smiles><smiles>Cc1ccc(C#Cc2ccc3c(=C(C#N)C#N)c4ccc(C#Cc5ccc(C(C)(C)C)cc5)cc4c(=C(C#N)C#N)c3c2)cc1</smiles>

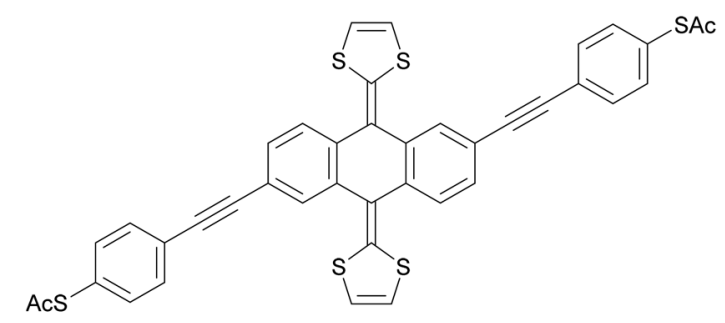<smiles>CC(C)(C)c1ccc(C#Cc2cc3c(s2)C(=O)c2cc(C#Cc4ccc(C(C)(C)C)cc4)sc2C3=O)cc1</smiles>

Fig. 21 Structures of molecular wires that incorporate a quinoidal unit into an OAE-like backbone.

based on other central units, such as benzodithiophene (e.g. 35). ${ }^{66}$

Various other functionality has been used to impart switching behaviour into OAE derivatives (Fig. 22). Examples of redox-active switches include the tetrathiafulvalene (TTF) wire 36, although conductance studies investigated only its ground state. ${ }^{218}$ The phenothiazine wire 37 was shown to have an impressive $c a$. 200-fold increase in conductance upon oxidation to a radical cation with trifluoroacetic acid, ${ }^{219}$ although the reversibility of this process was not commented upon. True OAEs containing arylene units such as naphthalenediimide (NDI), ${ }^{220}$ catechol $^{221}$ and benzodifuran ${ }^{143}$ have also been investigated as redox switches.

The photoswitching of diarylethenes such as $\mathbf{3 8}$ has been studied in both single-molecule ${ }^{222}$ and ensemble junctions. ${ }^{223,224}$ The conjugated, closed state $(\mathbf{3 8}-\mathbf{h})$ is around an order of magnitude more conductive than the open state (38-1). ${ }^{222,223}$ The norbornadiene-based wire 39 behaves as a memristor with two states that could be accessed by using current-induced local heating in one direction and single electron transfer in the other. ${ }^{225}$ Again, switching from a conju-

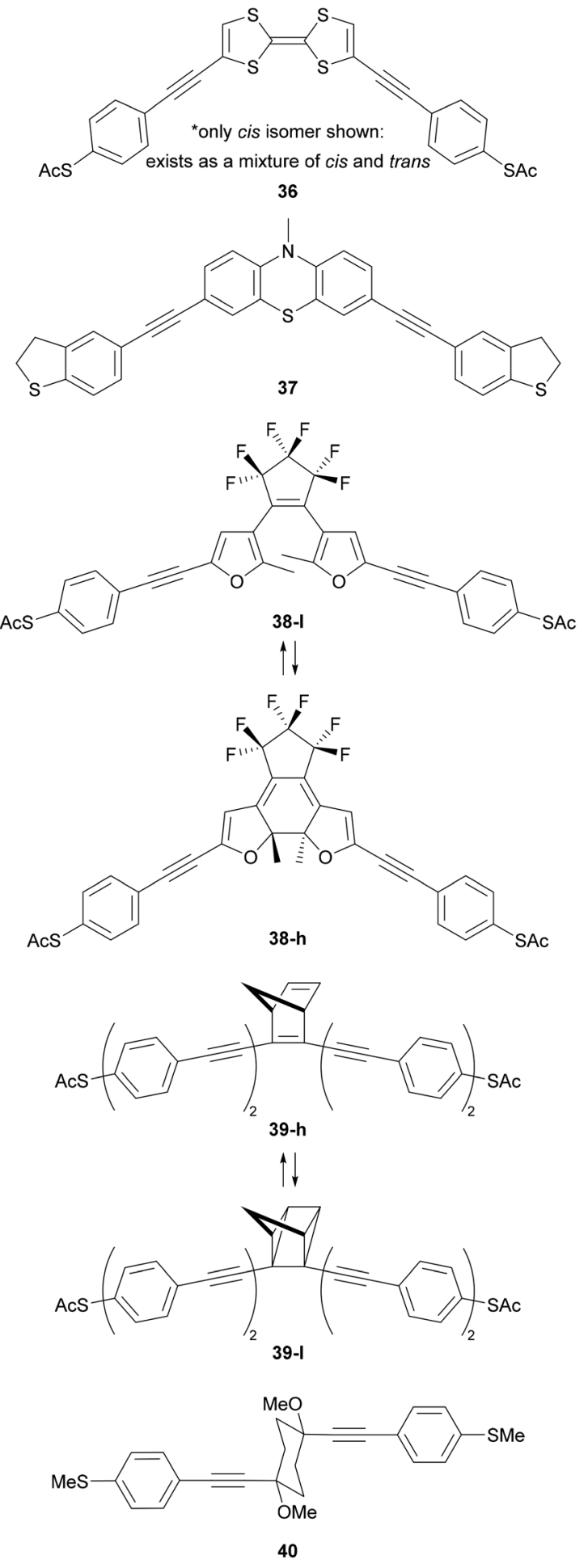

Fig. 22 OAE-like wires with switchable central units. High (h) and low (l) conductance forms of $\mathbf{3 8}$ and $\mathbf{3 9}$ are shown.

gated (39-h) to a non-conjugated (39-1) state reduced conductance by around an order of magnitude. ${ }^{225}$ The interconversion of conformational isomers of a cyclohexane ring in OAE3-like wires such as $\mathbf{4 0}$ has been detected in conductance measurements using the STM-BJ technique. ${ }^{226}$ 

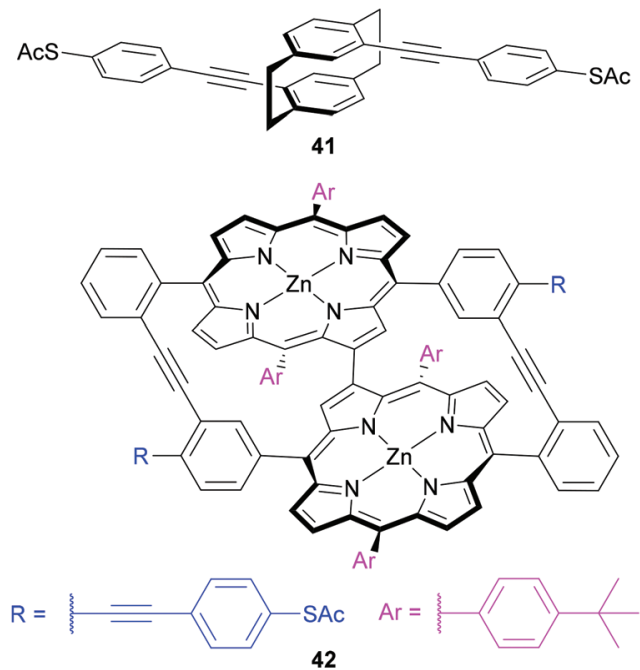

42

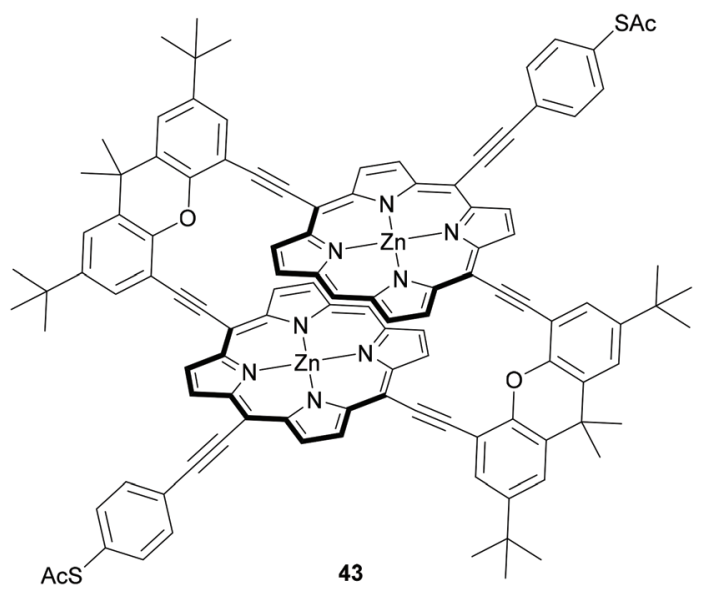

Fig. 23 OAE-like wires for investigation of mechanosensitive conductance.

Central units have also been designed to investigate mechanical switching of OAE-like wires (Fig. 23). Mechanosensitive conductance varying over an order of magnitude was observed for a para-cyclophane centred wire (41) by carefully controlling an MCBJ. ${ }^{227}$ This behaviour was attributed to QI effects related to the energy and relative phase of the frontier orbitals in the cyclophane unit, which are sensitive to the relative position of the two overlapping benzene rings. ${ }^{227}$ Initial synthetic attempts to improve on this design using a porphyrinbased cyclophane afforded instead an unusual triply-linked bowlshaped system (42), which was nevertheless subjected to conductance investigations. ${ }^{228}$ A change of approach gave a doublylinked porphyrin cyclophane (43), for which initial conductance results were recently reported. ${ }^{116}$ These molecular designs are a logical progression based on similar mechanosensitivity observed in junctions containing $\pi$-stacked OPE dimers. ${ }^{229}$ The use of cyclophanes ensures the overlapping $\pi$-systems are permanently held in place covalently, rather than only through transient supramolecular interactions.

The range of switching mechanisms discussed above emphasises that switching and sensing remains a hot topic in molecular electronics. Further innovative developments of functional OAEs which are responsive to diverse stimuli are easily anticipated, and could lend themselves to applications such as logic gates.

Central units in OAE-like wires have been selected for various other properties (Fig. 24). Biphenylene ${ }^{136}$ (e.g. 44) and dibenzopentalene ${ }^{230}$ have served to investigate the effect of antiaromaticity on molecular conductance (see section 3.5). Cyclopentadiene (e.g. 8d) has been used for comparison with 5-membered heterocycles 8a-c (see Fig. 10 and section 3.4). ${ }^{118,133}$ The photophysical properties of NDI derivatives have been utilised in molecular wire $\mathbf{4 5}$, which has broken conjugation. 45 is electroluminescent in a CNT-molecule-CNT junction architecture. $^{77}$

2.2.5 Organometallic analogues. In addition to purely organic species, molecular wires that combine OAE-type backbones with organometallic functionality are of interest. These compounds, where the metallic species forms part of the conductive backbone, are distinct from the true OAEs containing ligating moieties discussed in section 2.2.1 (Fig. 13). This section does not aim to cover organometallic approaches exhaustively, rather it will highlight some molecular designs of interest and comment on the effect of some key structural features. The interested reader is directed to a recent review concerned with molecular wires based on metal bis(acetylide) complexes (i.e. analogues of OAEs in which at least one aryl unit is replaced by a metal bearing auxiliary ligands). ${ }^{231}$<smiles>CSc1ccc(C#Cc2ccc3c(c2)-c2ccc(C#Cc4ccc(S(C)(=O)=O)cc4)cc2-3)cc1</smiles><smiles>C#CC1=CC=C(C#Cc2ccncc2)C1(C)C(=O)C#Cc1ccncc1</smiles><smiles>[R][CH]Oc1cc(C#CC(C)(C)C)c(O[GaH])cc1C#Cc1cc2ccccc2c2ccccc12</smiles>

Fig. 24 Example structures of OAE-like wires containing central functional units that disrupt or lack aromaticity or conjugation. 
Fig. 25 shows several OAE and OAE-like wires including organometallic functionality. Wire $\mathbf{4 6}$ and analogues with longer and shorter OAE units were used to show that the incorporation of ferrocene units into the backbone enhanced conductance relative to analogous OPEs. ${ }^{232}$ True OAEs, where one of the cyclopentadienyl ligands of a ferrocene moiety acts as an arylene unit in the OAE backbone, such as 47 , have also been studied, ${ }^{233}$ but a direct comparison with species resembling $\mathbf{4 6}$ does not yet appear to have been made.

Many metal bis(acetylide) complexes have been investigated in molecular junctions. ${ }^{231}$ Ruthenium and platinum com-
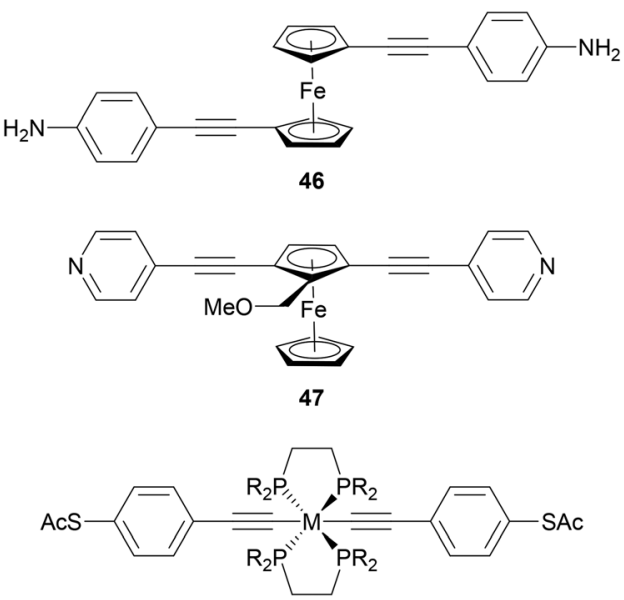

48a: $M=R u, R=P h ; 48 b: M=F e, R=E t ; 48 c: M=M o, R=P h$

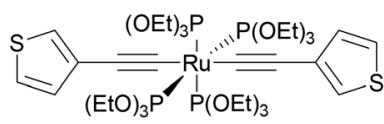

49

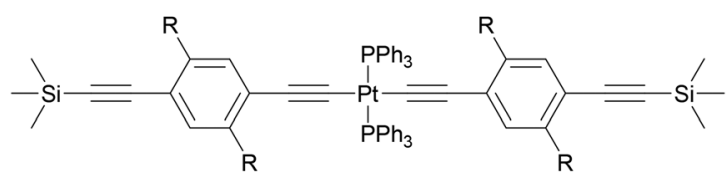

50a: $\mathrm{R}=\mathrm{H} ; \mathbf{5 0 b}: \mathrm{R}=\mathrm{OC}_{6} \mathrm{H}_{13}$
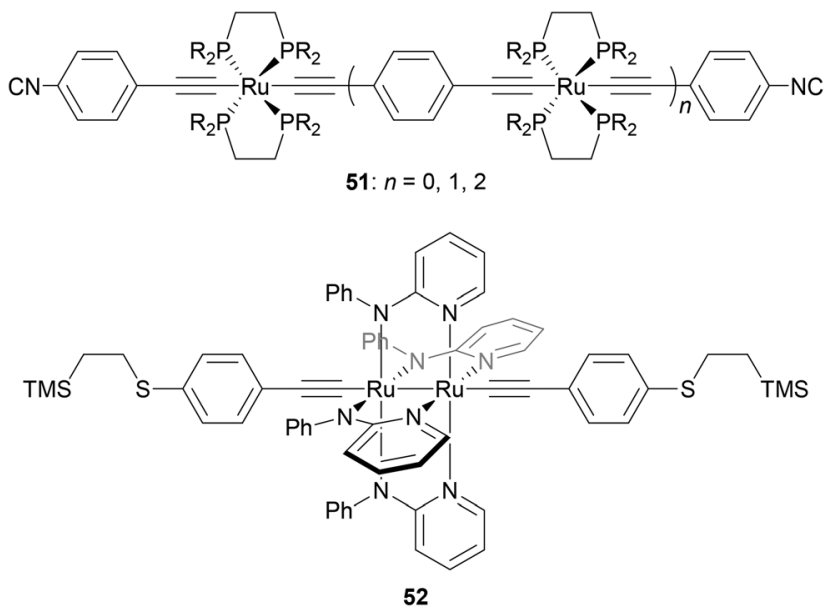

Fig. 25 Selected OAE-like wires containing organometallic functionality. plexes (e.g. 48a, 49, 50) are most widely-studied but other metals such as iron and molybdenum have also been used (e.g. 48b and 48c). ${ }^{231,234}$ Attractive properties of metal bis(acetylide) complexes include the possibility to finely tune molecular orbital energies through choice of metal and ancillary ligand, and opportunities to explore easily accessible redox states associated with transition metal centres. ${ }^{231}$ Conjugation is maintained across the metal centre because the metal $\mathrm{d}$-orbitals have the correct $\pi$-type symmetry to interact with the alkynyl $\pi$-orbitals.

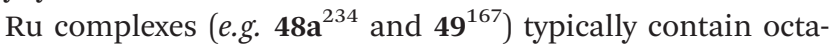
hedral metal centres bearing two bidentate or four monodentate phosphine (or phosphite) ligands in addition to the acetylide units. Pt complexes (e.g. $\left.\mathbf{5 0}^{235}\right)$ adopt a square planar geometry with two monodentate ancillary phosphine ligands. Comparisons between metal bis(acetylide) complexes and analogous OAEs give mixed results regarding the effect of replacing an aryl ring (usually benzene) with a metal centre. The extent of variation appears to relate to both the metal and the anchoring units. A Ru complex with SAc anchors was $c a .5$ times more conductive than the corresponding $\mathrm{OPE},{ }^{236}$ while a similar species with TMSE anchors was only around twice as conductive. ${ }^{171}$ In contrast, for Pt complex 50b, with TMSE anchors, comparable conductance to the OAE analogue was reported. ${ }^{235}$ With 3-thienyl anchors, the conductance of two $\mathrm{Ru}$ complexes with different ancillary ligands (e.g. 49) was similar to that of both Pt complex and OAE analogues. ${ }^{167}$ Using SMe or DMBT anchors, replacing the central benzene ring of an OPE3 with a Ru centre increased conductance by a factor of $c a$. 3-5 (dependent on anchor and ancillary ligands) whereas replacement with a Pt centre reduced conductance by more than a factor of $2 .{ }^{166}$

Similarly to all-organic wires, side groups can play an important role in organometallic systems. For example, hexyloxy chains hindered undesired additional anchoring interactions between an STM tip and $\mathrm{PPh}_{3}$ supporting ligands in a study of Pt bis(acetylide) complexes. ${ }^{235}$ The undesired throughligand conductance pathway dominates for 50a, whereas the expected pathway between the two TMSE anchoring groups is observed for 50b. Replacing the $\mathrm{PPh}_{3}$ ligands with $\mathrm{PEt}_{3}$ also prevents this interaction, showing that the choice of ancillary ligand can be important. ${ }^{235}$

Multimetallic systems have also been investigated because they offer the potential for enhanced redox response, amongst other properties. These include oligomeric species containing multiple metal centres, such as members of the isocyanoanchored series $\mathbf{5 1},{ }^{237}$ and diruthenium complexes such as 52. ${ }^{188}$ Multimetallic species have been combined with photoswitchable units to give highly functional OAE-like wires. ${ }^{238}$

\section{Electrical conductance of OAEs: structure-property relationships}

This section focuses on conductance studies that examine structure-property relationships in OAEs. The examples have 
been chosen to emphasise how the variety of structures available has enabled a detailed understanding of molecular conductance properties to be developed. The influence of factors including molecular length, connectivity, (cross-)conjugation, (anti)aromaticity and heteroatoms on molecular conductance will be discussed and, where relevant, related to QI.

\subsection{Impact of molecular length on conductance}

The conductance, $G$, of a series of oligomeric molecular wires can be related to molecular length, $L$, through the relationship:

$$
G=G_{\mathrm{c}} \mathrm{e}^{-\beta L}
$$

where $G_{\mathrm{c}}$ is the effective contact conductance, i.e. a term related to the properties of the anchoring group and electrodes. The attenuation factor, $\beta$, is primarily a property of the backbone and, in general, is lower for conjugated systems than non-conjugated systems. This relationship was shown to apply to $\mathrm{Au}-$ molecule-Au junctions in the early stages of molecular conductance studies ${ }^{54}$ and has been established for many molecular backbones. ${ }^{46} \beta$ is usually positive, i.e. conductance falls as molecular length increases. However, unusual negative values of $\beta$ were reported for porphyrin tapes ${ }^{105}$ and cumulenes. ${ }^{104}$ Related attenuation factors are observed for electron transfer in D-B-A systems and magnetic exchange coupling in biradical systems. ${ }^{78}$ However, these values are not directly comparable because different exchange interactions and tunnelling energy barriers will exist in the backbones under the very different experimental conditions. ${ }^{239}$

Many studies have experimentally determined values of $\beta$ for OAEs. ${ }^{60-62,112,124,125,128,172,176,214,240}$ Table 2 shows some of these values together with the structures of the oligomeric series and the junction type used to determine them. Although most of the examples use a similar para-linked OPE repeat unit, the $\beta$ value varies between 0.5 and $4.2 \mathrm{~nm}^{-1}$ using different anchoring groups and methods. Insightfully, two of the studies examined a sufficient range of oligomer lengths that a distinct reduction in $\beta$ ( $c a .1$ order of magnitude) was observed at molecular lengths beyond $2.5-3 \mathrm{~nm}$, as seen in Fig. 26. ${ }^{112,128}$ This change corresponds to a transition in the charge transport mechanism from tunnelling at short molecular lengths to hopping at longer molecular lengths. A related study of ferrocene-centred OAE-like species (e.g. 46, Fig. 25) found a similar transition and reported $\beta=2.2 \mathrm{~nm}^{-1}$ for the shorter species (albeit based on only two data points). ${ }^{232}$ Unlike the other studies discussed, which are concerned only with members of homologous series, Kaliginedi et al. combined an OPE oligomer series with OAE3s in which the central aryl unit was varied from benzene to naphthalene to anthracene. ${ }^{125}$ 2,6-Substituted naphthalene and anthracene derivatives (included in Table 2, entry 6) lay on the same trend line as the OPEs and were included when determining $\beta$, but 1,4substituted naphthalene (11) and 9,10-substituted anthracene $(\boldsymbol{p}$-23) did not and were excluded from the calculation of $\beta$ (for further discussion see section 3.3).

\subsection{Connectivity and quantum interference}

The discussion at the end of the previous section indicated that the connectivity of an aromatic unit within an OAE can have significant impact on its conductance. This can be attributed to the effects of QI. QI is the source of many differences between molecular and macroscale electronics. The nanoscale conductance pathways through a molecular wire are subject to quantum effects, and electrons propagating through different pathways can interfere with one another constructively (CQI) or destructively (DQI), depending on their phase. ${ }^{31}$

Studies described in section 2.2.1 showed that for benzene rings, para- (and ortho-) connectivity results in higher conductance than meta-connectivity. ${ }^{132,151,152,155-157}$ This archetypal demonstration of QI is rationalised in Fig. 27 for para- and meta-linked OAE3s with thienyl anchoring groups ( $\boldsymbol{p}$-53 and $\boldsymbol{m}$ 53, Fig. 27a). ${ }^{156}$ Fig. 27b shows the calculated transmission functions of the two wires, which can be separated into HOMO and LUMO contributions as seen in Fig. $27 \mathrm{c}$ and $\mathrm{d}$ for $\boldsymbol{p}$-53 and $\boldsymbol{m}$-53, respectively (higher- and lower-energy orbitals also contribute to a lesser extent, but are discounted for simplicity). Importantly, the HOMO and LUMO contributions have both an amplitude and a phase. In the case of $\boldsymbol{p}$-53, the phase of transmission is the same for both orbitals within the HOMOLUMO gap, so CQI takes place and $T(E)$ (and therefore conductance) is high around $E_{\mathrm{F}}$. Conversely, for $\boldsymbol{m} \mathbf{- 5 3}$, a phase difference of $\pi$ between the transmission through the HOMO and the LUMO in the HOMO-LUMO gap results in DQI, ${ }^{156}$ meaning $T(E)$ (and therefore conductance) is low around $E_{\mathrm{F}}$. The relationship between para- and meta-type connectivity with CQI and DQI, respectively, has also been demonstrated in OAEs containing larger aryl units such as fluorene. ${ }^{137,138}$

For larger aromatic systems the number of possible pathways increases and QI behaviour necessarily becomes more complex. For polycyclic aromatic hydrocarbons, QI behaviour can be qualitatively predicted using e.g. graphical methods ${ }^{34}$ or magic ratio rules. ${ }^{117,126}$ More quantitative predictions rely on the calculation of transmission functions ${ }^{31,32}$ (see Fig. 6 and section 1.2). The relationship between connectivity, QI and transmission functions was demonstrated experimentally in a comparison of para- and meta-linked OPE3s under external gating. ${ }^{157}$ Gating shifts the energy of the system away from $E_{\mathrm{F}}$, and therefore should allow access to more conductive regions of the transmission function for molecules that display DQI. In contrast, where CQI occurs $T(E)$ shows little variation until energies close to the molecular orbitals are reached. Indeed, as the gating voltage was varied the conductance of the meta-OPE3 spanned more than 2 orders of magnitude, whereas the conductance of the para-OPE3 varied by only a factor of $3 .{ }^{157}$

Polycyclic aromatics have been used to demonstrate different extents of CQI derived from connectivity. For a pair of anthanthrene-centred OAE3s (54 and 55, Fig. 28), 6,12connectivity (54, red) was predicted by magic ratio rules to be 81 times more conductive than 4,10-connectivity (55, blue). MCBJ measurements showed excellent agreement, with a conductance ratio of $c a .79$ at maximum junction extension. ${ }^{117}$ 
Table 2 Structures of OAE oligomer series used to determine attenuation factor, $\beta$, via different experimental methods

\begin{tabular}{|c|c|}
\hline OAE structure & $\beta / \mathrm{nm}^{-1}$ and method \\
\hline & 2.0 (OPE1-OPE3), 0.30 (OPE4-OPE7), ${ }^{128}$ STM-BJ \\
\hline
\end{tabular}

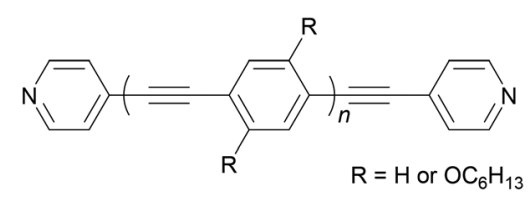

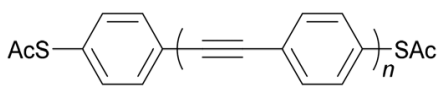

(a) 2.1 (OPE1-OPE3), ${ }^{240}$ c-AFM
(b) 3.7 (OPE2-OPE4), ${ }^{214}$ c-AFM
(c) 3.2 (OPE1 ${ }^{a}$-OPE3)/1.1 (OPE2 and OPE3 only), ${ }^{176}$ STM-BJ
(d) 2.3 (OPE2-OPE4), ${ }^{62}$ EGaIn
(e) 1.5 (OPE1 ${ }^{a}$-OPE3), ${ }^{124}$ Large area Au-molecule-PEDOT:PSS ${ }^{b}$<smiles>CC(C)(C)[As]c1ccc(C#Cc2ccccc2)cc1</smiles>

2.3 (OPE2-OPE4), ${ }^{62}$ EGaIn<smiles>CC(C)(C)c1ccc(C#Cc2ccc(C#N)cc2)cc1</smiles>

3.0 (OPE1-OPE3), ${ }^{61} \mathrm{Hg}$-Drop

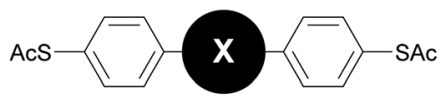

3.4 (OPE2-OPE5 + two OAE3s), ${ }^{125}$ STM-BJ and MCBJ
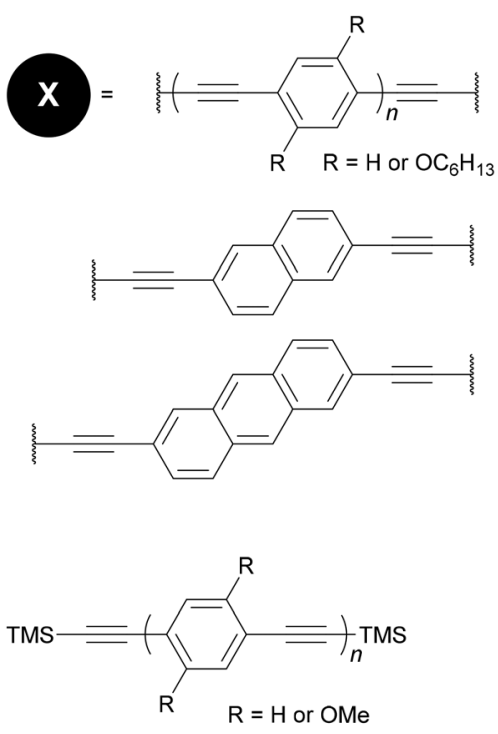

3.3 (OPE1-OPE3), ${ }^{172}$ MCBJ

(in situ deprotection, $\mathrm{C} \equiv \mathrm{C}$-Au bonding)

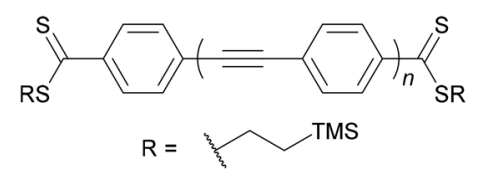

0.5 (OPE1-OPE3), ${ }^{176}$ STM-BJ 
Table 2 (Contd.)

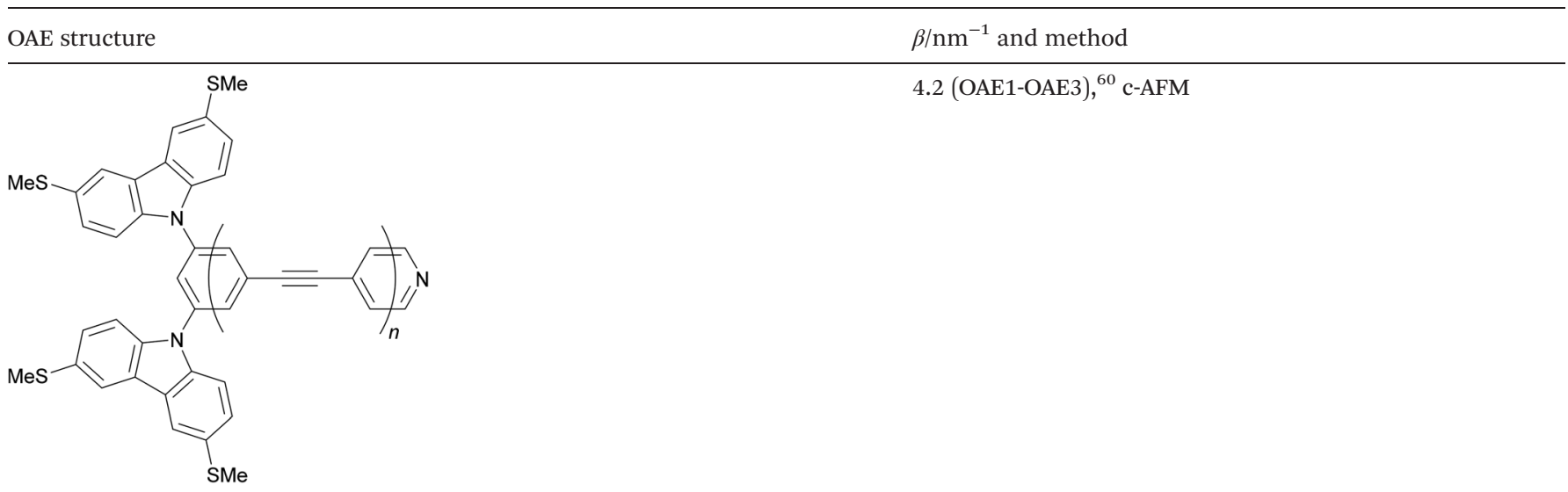

${ }^{a}$ For OPE1, benzenedithiol was used rather than the acetyl-protected species. ${ }^{b}$ PEDOT:PSS = poly(3,4-ethylenedioxythiophene) polystyrene sulfonate, a conductive polymer.
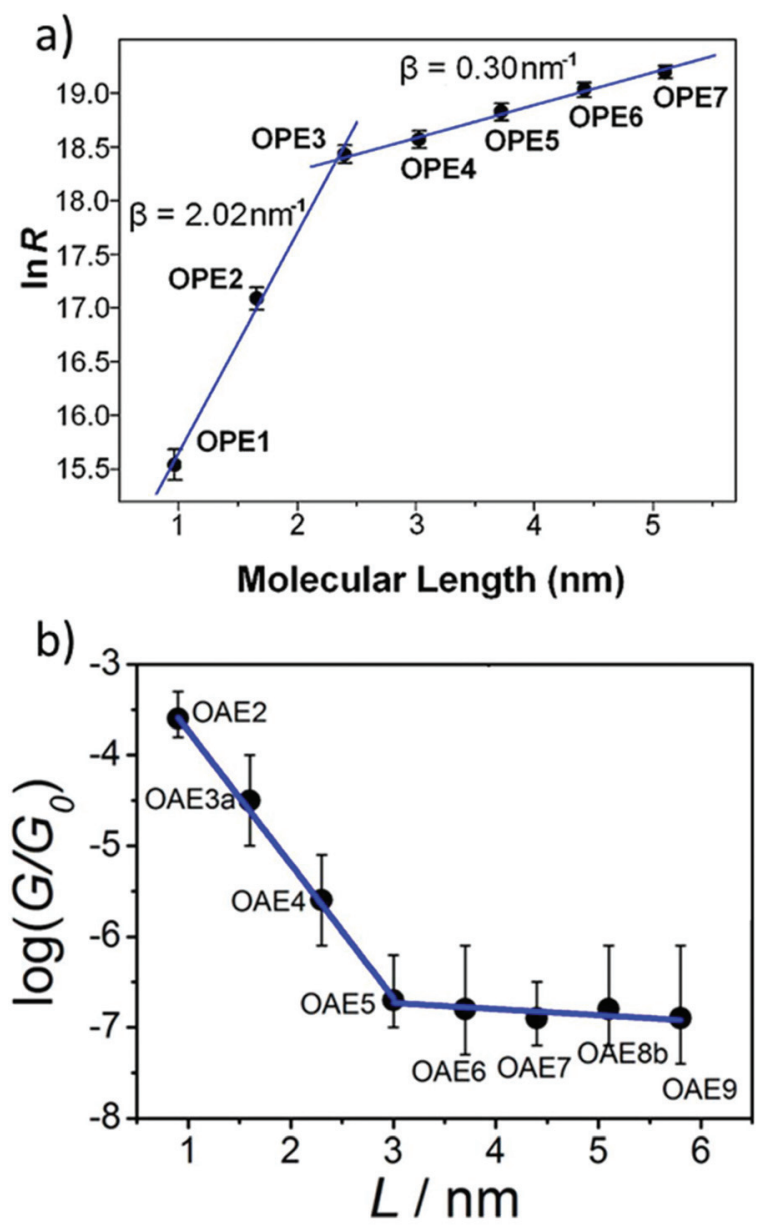

Fig. 26 Plots illustrating a transition of $\beta$-value with increasing molecular length for: (a) amine-anchored OPEs (entry 1, Table 2); this figure has been adapted from ref. 128 with permission from the American Chemical Society, copyright 2009; (b) pyridine-anchored OAEs (entry 2, Table 2); this figure has been adapted from ref. 112 with permission from the American Chemical Society, copyright 2013. Note that in plot (a) the $y$-axis represents resistance (i.e. the inverse of conductance) and hence the sign of the slopes differs between the two graphs.
Similar behaviour was observed in SAMs using isomeric anthracene-centred OAE3s (56 and 57, Fig. 28). ${ }^{126}$ 9,10Connectivity $(\mathbf{5 6}$, red) was predicted to be 16 times more conductive than 1,5-connectivity (57, blue). The experimental ratios determined using c-AFM were 10.2 (SMe anchoring group) and 14.3 (SAc anchoring group), in reasonable agreement with the prediction. ${ }^{126}$ The correlation between magic ratio rules and experiment in these cases is impressive, but it should be noted that to our knowledge they have only been shown to apply to all-hydrocarbon aryl units or heteroaryl units derived from bipartite lattices.

QI effects have also been observed in systems with multiple anchoring groups which might be expected to afford independent conductance pathways that interact additively. By comparing OAE2s with a para-pyridyl-, meta-amino- or 2-aminopyridylanchor (and a second para- or meta-pyridyl anchor) it was found that parallel conductance pathways do not interact additively (i.e. according to Kirchoff's law) if, in isolation, CQI is expected for one pathway and DQI for the other. ${ }^{185}$ Instead, the overall conductance of the parallel system was between that of the two isolated pathways. In contrast, where both pathways would be expected to exhibit DQI in isolation, Kirchoff's law appeared to hold. ${ }^{185}$

\subsection{Conjugation}

In section 3.1, it was stated that the molecular conductance of OAE3s with central 1,4-substituted naphthalene and 9,10-substituted anthracene units (13 and $\boldsymbol{p}$-23) did not lie on the same trend line as OPEs when investigating the $\beta$-value. ${ }^{125}$ An increase in conductance as the central ring system increased in size, while the length of the conductance pathway remained the same, was also observed by Liu et al. in an MCBJ-study of five acene-centred OAE3s with central aryl units ranging from benzene to pentacene (25a, 11, $\boldsymbol{p}$-23, 58 and 59, Fig. 29a). ${ }^{69}$ This behaviour relates to the conjugation of the central aryl unit. As the size of the acene, and hence conjugation, increases the HOMO-LUMO gap decreases. This, in turn, 
a)
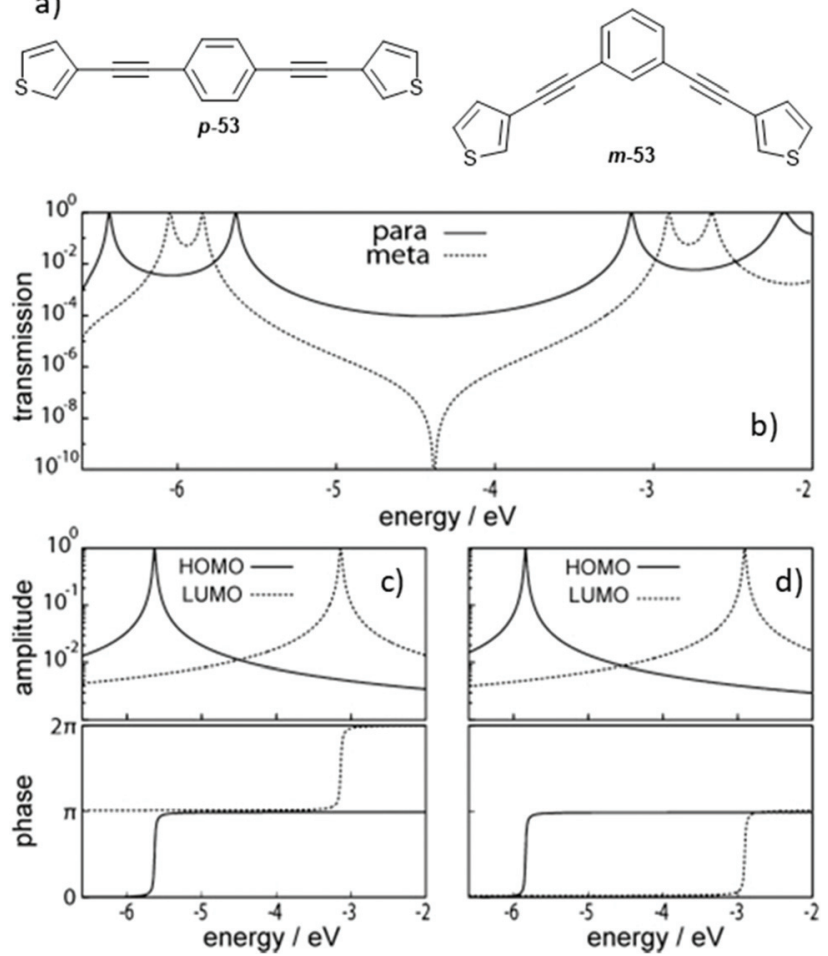

Fig. 27 Structures (a) and transmission functions (b) of para- and metaconnected OAE3s $p-53$ and $m-53$, together with the amplitude (top) and phase (bottom) of transmission through the HOMO and LUMO of $p-53$ (c) and $m-53$ (d). This figure has been adapted from ref. 156 with permission from Wiley-VCH, copyright 2013.

results in increased conductance as seen in the molecular transmission functions (Fig. 29b); the smaller HOMO-LUMO gap reduces the depth of the transmission minimum close to $E_{\mathrm{F}}{ }^{69}$ It is noteworthy that Liu et al. did not observe the same trend when SAMs of the molecules were measured using the EGaIn method. In this case conductance was fairly similar for all OAEs except for the pentacene system, for which it was significantly lower. ${ }^{69}$ This was attributed to intermolecular packing interactions between acene units in the tightly-packed SAM (which is also believed to be the source of lower-conductance features present in the MCBJ data). ${ }^{69}$

The impact of cross-conjugation on QI has been investigated by comparing aromatic systems to their quinoidal analogues. Linearly-conjugated 2,6-anthracene-centred OAE3 systems (e.g. 12) have been compared to cross-conjugated anthraquinone analogues (e.g. 32) in conductance studies using $\mathrm{MCBJ},{ }^{125}$ STM-BJ, ${ }^{67}$ c-AFM ${ }^{67,214}$ and EGaIn. ${ }^{65,67}$ Non-conjugated 9,10dihydroanthracene species (e.g. 60) are often also included. As shown in Fig. 30, anthracene systems are consistently the most conductive, followed by 9,10-dihydroanthracenes, then anthraquinones. For 2,6-connectivity, conductance therefore follows the trend of linear conjugation $>$ no $\pi$-conjugation $>$ cross-conjugation. Charge transport calculations illustrate that this can be related to DQI in the case of anthraquinone species. ${ }^{125,214}$

DQI is not a fundamental property of the anthraquinone unit; a comparison of 1,4- and 1,5-connectivities (where the
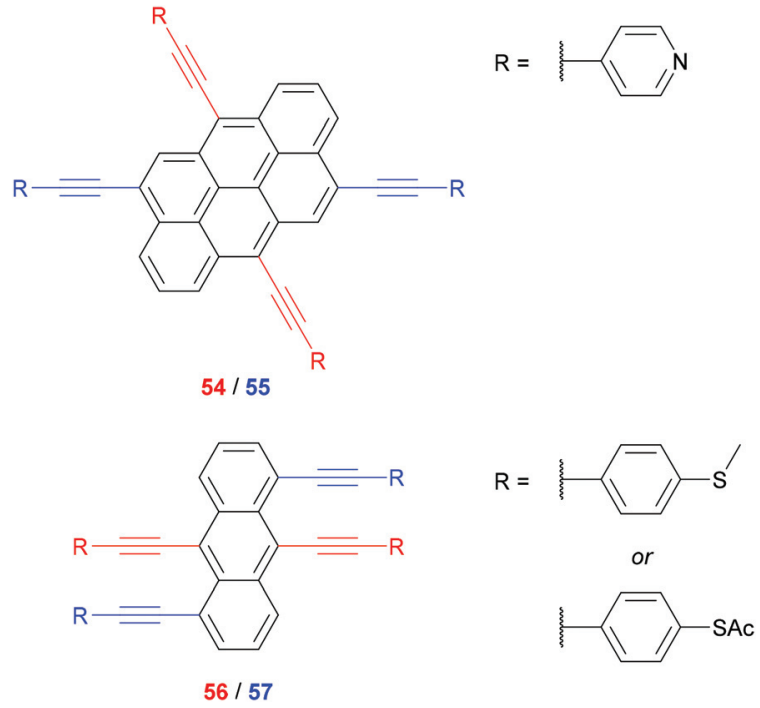

Fig. 28 Isomeric OAEs containing polycyclic aryl units which have been incorporated into OAE wires to compare different substitution patterns. For a given isomer, only either the red or blue substituents are present, the others are replaced by $\mathrm{H}$ (or solubilising $n$-octyloxy or 4-(2ethylhexyloxy)phenyl side chains for 54 and 55, respectively). The red and blue substitution patterns correspond to higher and lower conductance isomers, respectively.

conductance pathway respectively does or does not pass across the quinoidal ring, i.e. the wire is either linearly or cross-conjugated) shows no evidence of DQI in the 1,4-case. ${ }^{215}$ The conductance of the 1,5-species is much lower than the 1,4-analogue, which is attributed to DQI in the former (although the characteristic antiresonance is not seen in the calculated transmission function) ${ }^{215}$ Electrochemical reduction to the conjugated dihydroxyanthracene derivative increased conductance in both cases, but much more significantly for the 1,5-connectivity. This was related to the "switching off" of DQI in this case. ${ }^{215}$ Similar redox-switching behaviour has been reported for 2,6-connected anthraquinone ${ }^{217}$ and an anthraquinonederived extended TCNQ unit. ${ }^{63} \mathrm{~A}$ theoretical study indicated that the connectivity of cross-conjugated anthraquinones can influence QI behaviour; ${ }^{241}$ this observation has recently been rationalised using an approach based on curly arrows. ${ }^{34}$

Zhang et al. used OAE3s containing benzodithiophene (BDT) derivatives to investigate the relationship between conjugation and QI. ${ }^{66} \mathrm{~A}$ linearly-conjugated BDT (17) was compared to its quinone analogue (35) and an isomeric cross-conjugated BDT (61), alongside an anthraquinone species (32) for reference. Compared to linearly-conjugated $\mathbf{1 7}$, cross-conjugation alone was observed to reduce conductance much less than cross-conjugation due to a quinoidal system (Fig. 31). Calculated transmission functions showed that for the quinoidal systems a sharp DQI feature was present close to $E_{\mathrm{F}}$, whereas for the cross-conjugated isomer the equivalent antiresonance was significantly shifted towards the HOMO ${ }^{66} \mathrm{~A}$ key finding of this study is therefore that the nature of the functionality which imparts cross-conjugation has substantial 
a)

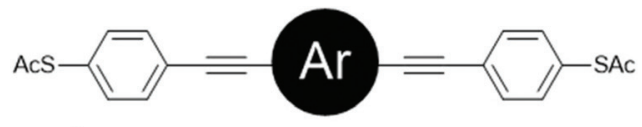<smiles>Ic1ccccc1</smiles>

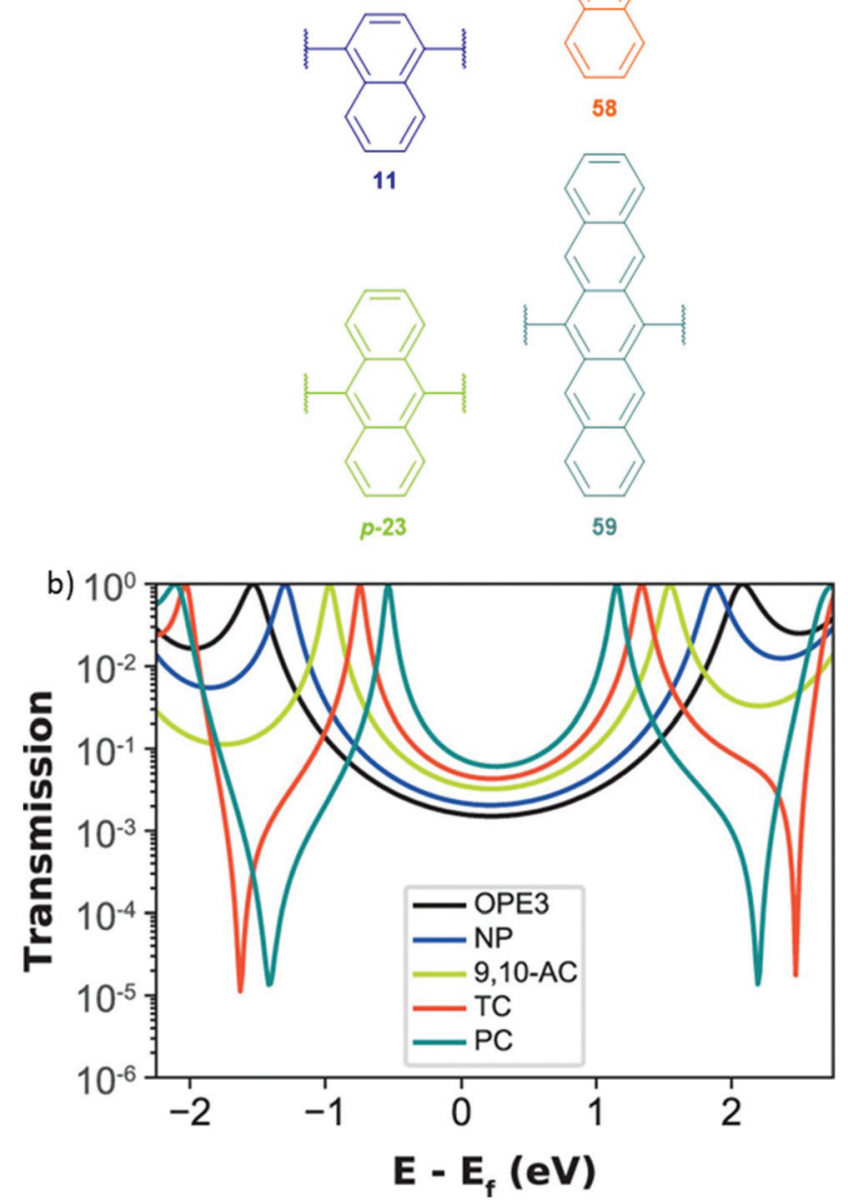

Fig. 29 (a) Structures of a series of thiol-anchored acene-centred OAE3s. The core acenes are: para-benzene (OPE3, 25a), 1,4-naphthalene (NP, 11), 9,10-anthracene (9,10-AC, $p$-23), 5,12-tetracene (TC, 58) and 6,13-pentacene (PC, 59); (b) Nonequilibrium Green's FunctionDensity Functional Theory (NEGF-DFT) based transmission functions for the molecules in (a). Panel (b) has been reproduced from ref. 69 with permission from the American Chemical Society, copyright 2020.

influence on the resulting QI behaviour. A study that examined a series of OAE3s with central anthraquinone-like species with different cross-conjugating groups also found considerable variation in conductance. ${ }^{67}$

Cross-conjugation has also been shown to supress the reduction in conductance associated with DQI. In a comparison of OAE3s with central dimethylfluorene or fluorenone units (62 and 63 in Fig. 32) para-connectivity (62, red) afforded higher conductance than meta-connectivity $(63$, blue $){ }^{138}$ However, the magnitude of this difference was significantly a)
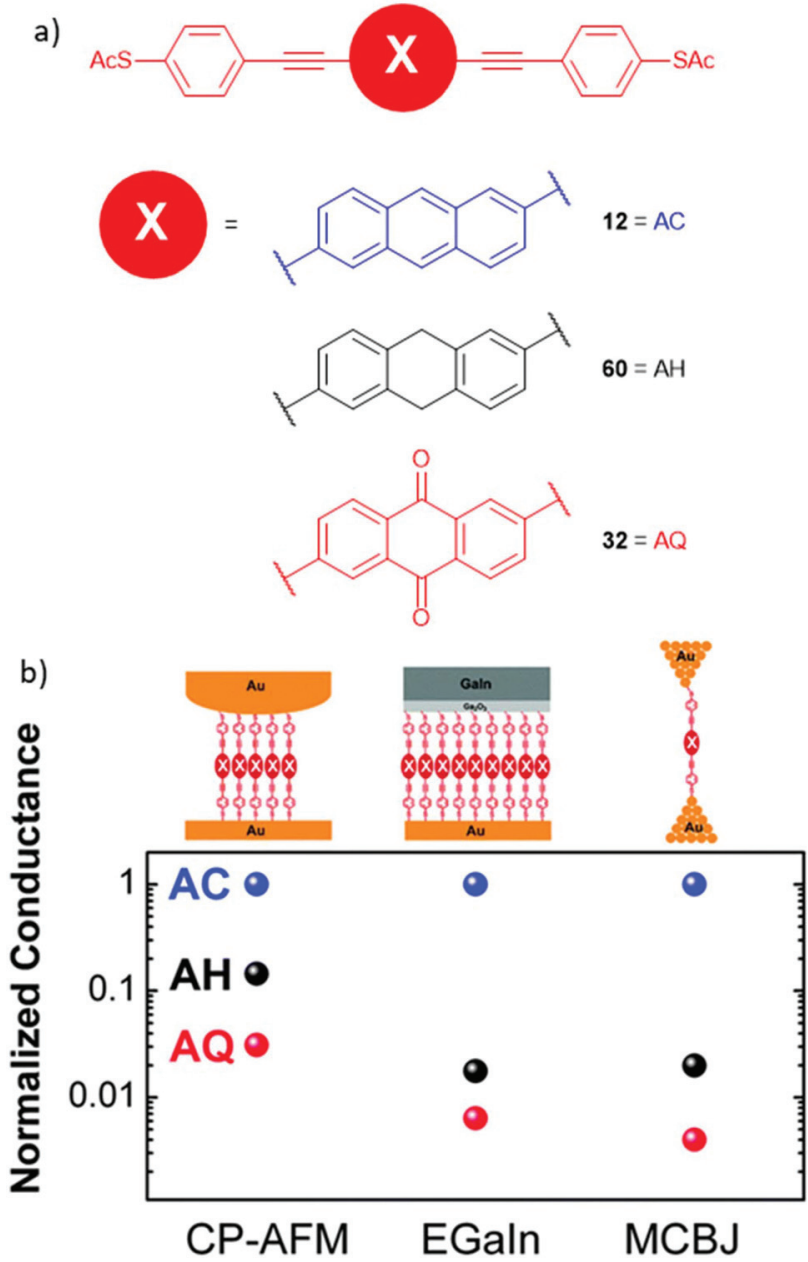

Fig. 30 Structures (a) and comparison of conductances (b) of thiolanchored OAE3 and OAE3-like species with central 2,6-connected anthracene (AC, blue, 14), 9,10-dihydroanthracene (AH, black, 60) and anthraquinone $(A Q$, red, 41) units determined using different experimental methods (CP-AFM = C-AFM). This figure has been adapted from ref. 214 with permission from the PCCP Owner Societies, copyright 2013. Some of the data included is originally from other works. ${ }^{65,125}$

lower for the fluorenone species $\mathbf{6 2} \mathbf{b}$ and $\mathbf{6 3 b}$ (2-3 times lower) than the dimethylfluorene species 62a and 63a ( $c a .2$ orders of magnitude lower). The carbonyl group of meta-linked fluorenone $\mathbf{6 3 b}$ is cross-conjugated with the anchoring groups, whereas there is no cross-conjugated functionality in the dimethylfluorene analogue 63a, suggesting that this small structural change is responsible for the drastically different conductance behaviour. ${ }^{138}$

\subsection{Aromaticity and heteroatom effects}

Heterocyclic systems have been used as arylene units in OAEs to investigate the effects of aromaticity on molecular conductance. For simple five-membered heterocycles, aromaticity increases in the order furan < pyrrole < thiophene; cyclopentadiene is a non-aromatic analogue that can easily be compared with this series. Chen et al. studied amine-anchored OAE3s 


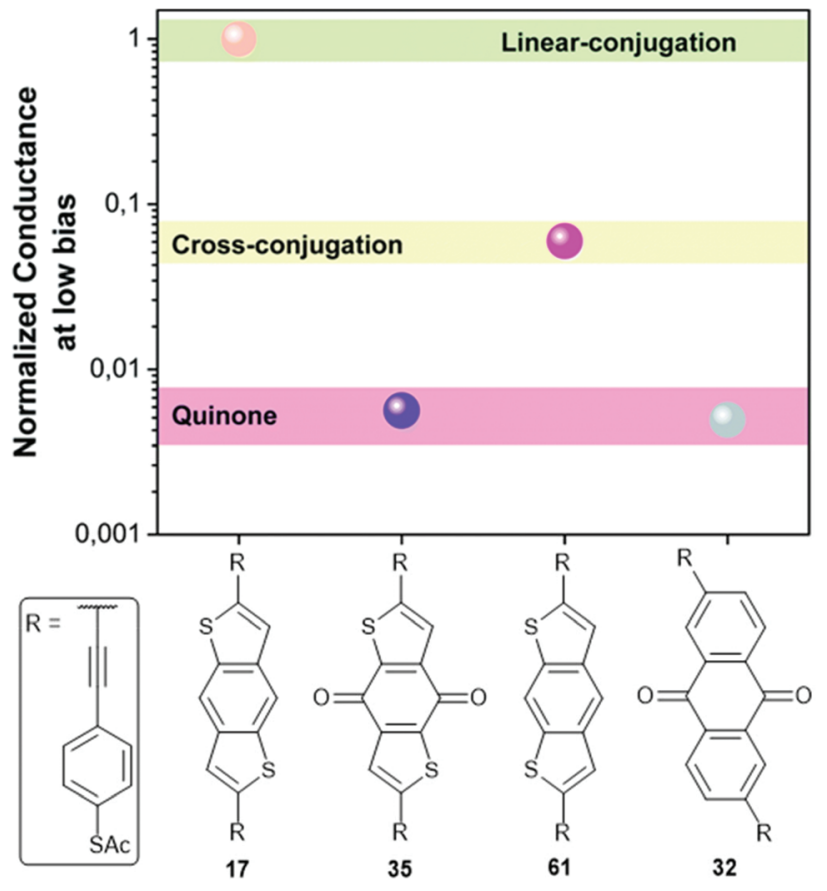

Fig. 31 Comparison of the conductance of three OAE3s incorporating BDT derivatives and an anthraquinone species measured using the EGaln method. This figure has been adapted from ref. 66 with permission from The Royal Society of Chemistry, copyright 2018.
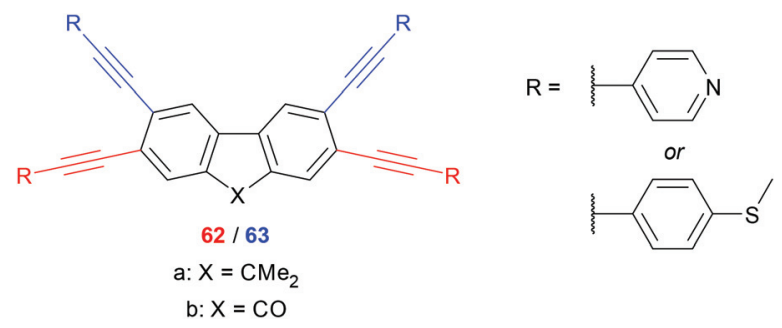

Fig. 32 Fluorene derivatives which have been incorporated into OAE wires to compare different substitution patterns. For a given isomer, only either the red or blue substituents, corresponding to higher and lower conductance isomers, respectively, are present, the others are replaced by $\mathrm{H}$.

with central thiophene, furan or dimethylcyclopentadiene rings (64b-d in Fig. 33) and found that conductance decreased as the aromaticity of the central ring increased. ${ }^{133}$ A later study by Yang et al. using pyridyl anchoring groups saw no variation with aromaticity for an analogous series of OAE3s also including a pyrrole derivative (8a-d in Fig. 33). ${ }^{118}$ The difference between the two studies was attributed to anchoring group effects. ${ }^{118}$ Contrasting results were observed with an asymmetric connectivity (65a-d in Fig. 33). In this case the pyrroleand furan-centred species were the most conductive, followed by the cyclopentadiene derivative, then the thiophene-centred OAE3. ${ }^{118}$ Conductance appeared to increase with the electronegativity of the varied group rather than relating to aromati-

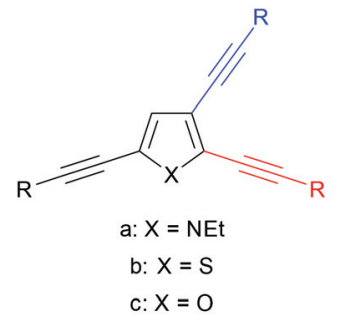

$\mathrm{d}: \mathrm{X}=\mathrm{CMe}_{2}$

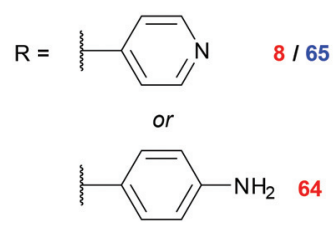

Fig. 33 Heterocyclic aryl units (and related species) which have been incorporated into OAE wires to compare different substitution patterns. For a given isomer, only either the red or blue substituents, corresponding to higher and lower conductance isomers, respectively are present, the others are replaced by $\mathrm{H}$.

city. ${ }^{118}$ The transmission functions of the asymmetric series also showed evidence of DQI ( $c f$. meta-connectivity).

A related study of "bridged biphenyl" species (15a-d, and 66a-d in Fig. 33) also explored the effects of aromaticity and heteroatoms on the conductance of OAE3s. ${ }^{137}$ For para-connectivity (15, red), conductance decreased as the aromaticity of the heterocyclic core increased. The overall trend in conductance was $\mathrm{X}=\mathrm{O}>\mathrm{NEt}>\mathrm{CMe}_{2}>\mathrm{S}$, meaning that the non-aromatic dimethylfluorene species (15d) did not follow the aromaticity trend. ${ }^{137}$ Contrasting results were obtained for meta-connectivity $(66$, blue) where conductance followed the trend $\mathrm{X}=$ $\mathrm{CMe}_{2} \approx \mathrm{NEt}>\mathrm{O} \approx \mathrm{S}$. Aromaticity alone cannot explain this conductance trend, and as for five-membered heterocycles ${ }^{118}$ electronegativity and QI appear to be important factors. ${ }^{137}$ In an alternative rationalisation, the heteroatoms can also be considered as cross-conjugating functionality ${ }^{34}$ ( $c f$. the discussion of the structurally-similar fluorenone-containing OAE 63b above $^{138}$ ). Additional evidence supporting an inversely proportional relationship between aromaticity and conductance is desirable, but these studies suggest it may prove difficult to conclusively separate the effects of aromaticity from associated factors.

Heteroatom effects have also been considered separately from aromaticity. The influence of heteroatoms, either within the OAE backbone or as pendent substituents, is minimal in the case of linearly-conjugated species for which CQI is expected to occur. $^{93,112,131,201}$ In contrast, clear effects are seen when heteroatoms are introduced into meta-linked OAEs for which DQI is expected. Liu et al. compared a meta-OPE3 to analogous OAEs in which the central ring is pyridine rather than benzene. ${ }^{132}$ Using the MCBJ method, they observed different conductance behaviour depending on the position of the nitrogen in the central ring (Fig. 34). When the nitrogen was meta to both alkynes (M1, Fig. 34), the conductance was comparable to the parent OPE3. 

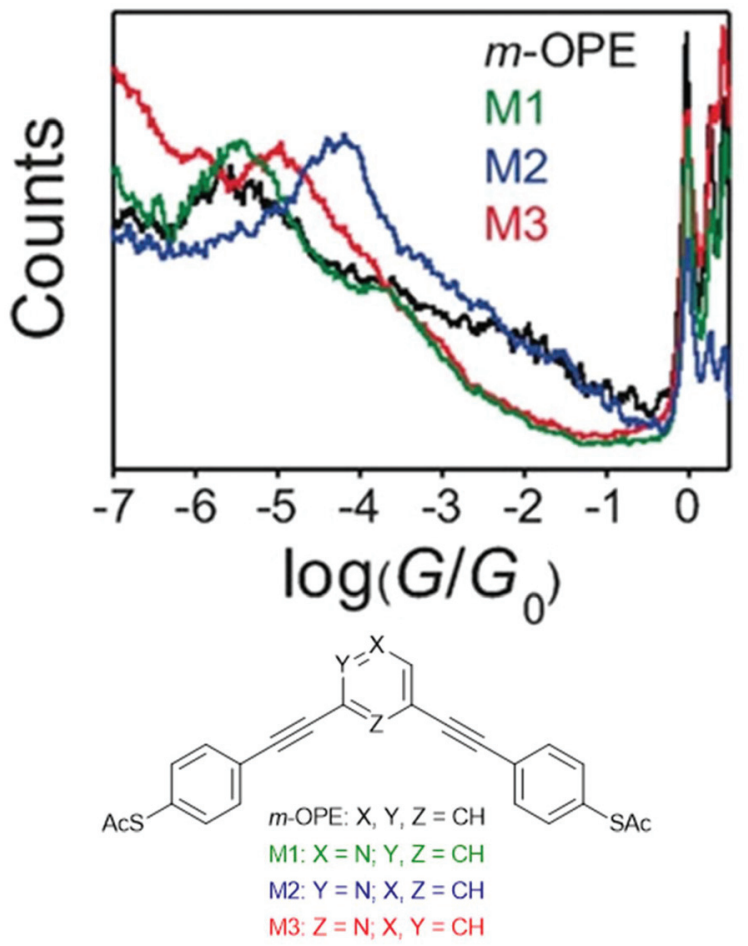

Fig. 34 MCBJ conductance histograms (top) and structures (bottom) for a series of meta-OAE3 molecular wires. This figure has been adapted from ref. 132 with permission from Wiley-VCH, copyright 2016.

The other two isomers (M2 and M3) each have higher conductance than the parent system. ${ }^{132}$

Comparable effects were seen in an investigation of metalinked OPE3s with methoxy-substituted analogues, ${ }^{153}$ indicating that pendent heteroatoms can also influence conductance. In this case, substitution in the mutually meta-position (relative to the alkynes) again afforded similar conductance to the parent system, whereas enhanced conductance was observed for substitution para to one alkyne and ortho to the other. ${ }^{153}$ In STM-BJ studies, substitution at the mutually ortho position did not afford an increase in conductance. However, computational studies showed this was a steric rather than an electronic effect; enhanced conductance was seen in the transmission function if the methoxy substituent was constrained to an unfavourable position in which its lone pair could interact with the OPE $\pi$-system. ${ }^{153}$

These $\mathrm{T}^{132,153}$ and other studies ${ }^{34-36}$ have related the increased conductance associated with the addition of heteroatoms to meta-linked OAEs to the electron-withdrawing or -donating properties of the heteroatom(s). Charge transport simulations showed that the presence of donor or acceptor groups at certain positions shifted DQI antiresonances away from $E_{\mathrm{F}}$, meaning $T(E)$ and hence conductance was higher in this region. ${ }^{35,36}$ Further experimental confirmation of these effects (including the scope of suitable functional groups), and analysis of their influence on measured molecular conductance would provide a means to better design new molecules which exploit these DQI effects. a)
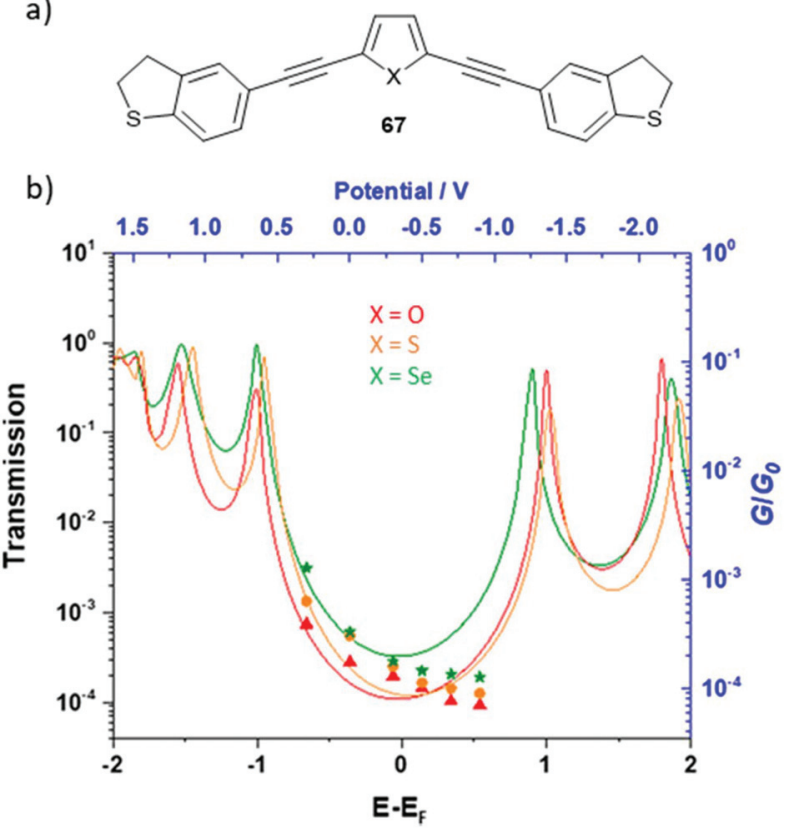

Fig. 35 (a) General structure of DHBT-anchored OAE3 wires 67 with a central heterocycle used to study gating effects on molecular conductance; (b) transmission functions (solid lines, black axes) and electrochemically gated STM-BJ conductance data (data points, blue axes) for OAE wires in (a) with $X=O, S$ and Se. Panel (b) has been adapted from ref. 134, with permission from the American Chemical Society, copyright 2021.

OAE3s with central five-membered heterocycles containing different chalcogens (67, Fig. 35a) were used in a study of gating effects on molecular conductance. ${ }^{134}$ Without gating, molecular conductance followed the trend $\mathrm{X}=\mathrm{O}<\mathrm{S}<$ Se. This was the inverse of the trend in HOMO-LUMO gaps for which $\mathrm{X}$ $=\mathrm{Se}<\mathrm{S}<0 .{ }^{134}$ When gating voltages ranging from -0.9 to $+0.3 \mathrm{~V}$ were applied, the range of possible conductance values followed the order $\mathrm{X}=\mathrm{O}<\mathrm{S}<\mathrm{Se}$, with those of the $\mathrm{X}=\mathrm{Se}$ species spanning an order of magnitude. The gated conductances showed reasonable agreement with the calculated transmission functions for the molecules, as shown in Fig. 35b. ${ }^{134}$ The observed variation of the gating effect with relatively small structural changes is pertinent to the design of molecular transistors.

\subsection{Antiaromaticity}

Aromatic systems have also been compared to related antiaromatic species. Gantenbein et al. compared different connectivities of biphenylene in OAE3s with structurally similar aromatic systems (Fig. 36). ${ }^{136}$ Wire 44, where the conductance pathway crosses the antiaromatic $4 \pi \mathrm{e}^{-}$central ring, was compared to OAE3s with central fluorene $(62, \mathrm{R}=$ para-(methylthio)phenyl) or biphenyl (68) units, i.e. with a non-aromatic or without any central ring. No significant conductance variation was seen between these three species. ${ }^{136}$ OAE3s with 1,4-connectivity across central biphenylene (69), naphthalene (70) and anthracene (71) units were also compared. In these cases the 


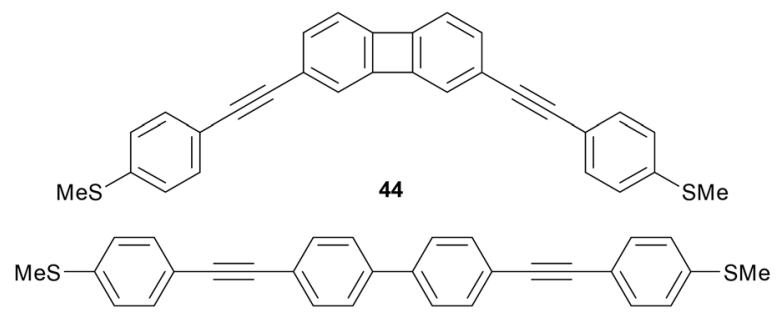

68
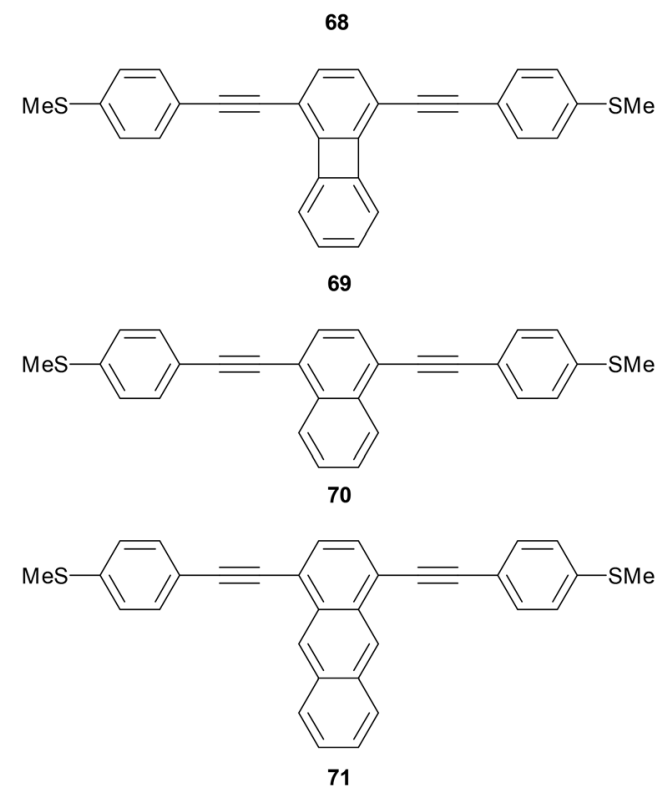

Fig. 36 Structures of biphenylene-containing molecular wires and selected reference species used to investigate the effect of antiaromaticity on molecular conductance.

fused (anti)aromatic ring(s) are pendent to the conductance pathway. Again, no significant conductance variation was observed. ${ }^{136}$ The limited effect of antiaromaticity in these cases could relate to the fusion of the antiaromatic cyclobutadiene system to aromatic benzene rings in biphenylene. This may reduce the effective antiaromaticity of the system as a whole.

To further investigate antiaromaticity, Schmidt et al. compared dibenzopentalene-centred OAE3s 72 and 73 with two aromatic model systems: a 9,10-anthracene-centred OAE3 (74) and an OPE3 (75) (Fig. 37). ${ }^{230}$ The $8 \pi \mathrm{e}^{-}$pentalene system at the core of dibenzopentalene is antiaromatic. The conductance pathways of $\mathbf{7 2}$ and model systems $\mathbf{7 4}$ and $\mathbf{7 5}$ are near-identical in length and comprise equivalent alternating single and multiple bonds. The conductance of these three systems is similar (Fig. 37), although that of 75 is slightly lower due to its larger HOMO-LUMO gap ( $c f$. the acene series ${ }^{69} 25 \mathrm{a}, 11, p-23,58$ and 59 in Fig. 29). ${ }^{230} 73$ has a longer conductance pathway but is significantly less conductive relative to 72 than would be expected based on this length difference alone. The conductance difference appears to relate to cross-conjugation rather than the antiaromaticity of dibenzopentalene, as supported by the presence of an antiresonance in the transmission function of $73 .{ }^{230}$ Small antiaromatic systems fused to aromatic rings
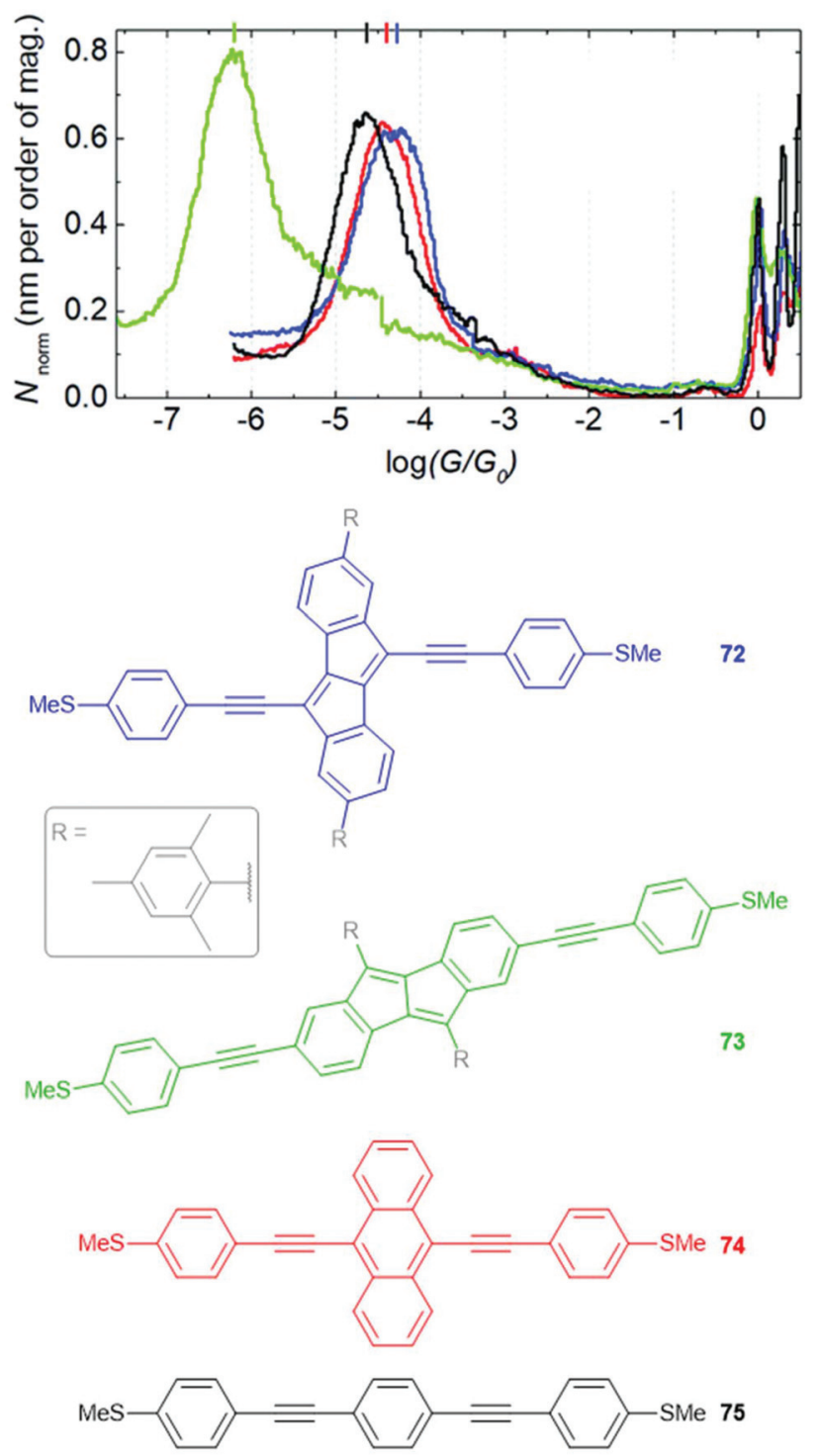

Fig. 37 STM-BJ 1D Conductance histograms (above) and structures (below) of OAE3 wires used by Schmidt et al. to investigate the effects of antiaromaticity on molecular conductance. The structures are coloured to match the corresponding histogram trace. This figure has been adapted from ref. 230 with permission from the Royal Society of Chemistry, copyright 2021.

appear to have only minimal influence on molecular conductance.

\subsection{Anchoring groups}

Comparisons between anchoring groups are not always straightforward as they may rely on results obtained in different laboratories using different experimental equipment and methods. ${ }^{158}$ However, multiple studies have specifically examined the effect of changing the anchoring group of otherwise identical OAEs. Reported trends include:

1. Conductance of OAE2s varies with anchoring group in the sequence $-\mathrm{SAc}>-\mathrm{NH}_{2}>$ pyridyl $\gg-\mathrm{CN}$, using STM-BJ and 
MCBJ. Thiol and pyridyl groups gave the most stable junctions, and showed the highest junction formation probability. ${ }^{160}$

2. Based on values for OAE2s in a study of oligoynes, the trend for conductance with anchoring group is DHBT $>-\mathrm{SAc}>$ $-\mathrm{NH}_{2}>$ pyridyl $\gg-\mathrm{CN}$, using STM-BJ and MCBJ. DHBT, thiol and pyridyl groups all showed high junction formation probability. ${ }^{102}$

3. A series of alkyne-anchored OPEs were more conductive than their thiol analogues in an MCBJ study. ${ }^{172}$

4. A study of OAE3s found conductance followed the trend $-\mathrm{SAc}>-\mathrm{SMe} \approx-\mathrm{NH}_{2}>$ pyridyl, whereas a study of junction lifetimes gave the trend pyridyl $>-\mathrm{SAc} \approx-\mathrm{NH}_{2}>-\mathrm{SMe}^{161}$

5. Solvent effects on the conductance of OAE2 wires varied with anchoring group in MCBJ measurements. With -SMe anchors, conductance increased by almost an order of magnitude when the solvent was changed from mesitylene to THF, whereas smaller changes were seen for -SAc or pyridyl anchors. $^{242}$

6. The effect of copper complexation on the conductance of phenanthroline-centred OAE3s 21a-c (Fig. 13) differed depending on the anchoring group in "self-breaking" MCBJ experiments. ${ }^{148}$ For the free wires, conductance followed the trend SAc $>$ alkyne $>$ pyridyl. Upon Cu-complexation, the conductance of the thiol-anchored OAE3 fell slightly, while that of the alkyne-anchored OAE3 fell by an order of magnitude. In contrast, the conductance of the pyridine-anchored OAE3 increased by a factor of $\mathrm{ca}$. 6. The different responses were attributed to the dominance of different molecular orbitals on conductance behaviour, as complexation changes orbital energies. $^{148}$

Anchor groups influence which molecular orbital dominates the conductance properties of a molecular wire (i.e. whether the HOMO or LUMO is closer in energy to $E_{\mathrm{F}}$ in the transmission function, see Fig. 6). This phenomenon is of particular relevance to the thermoelectric properties of molecular wires and is discussed further in section 4.1.

\section{Thermoelectric properties of OAEs}

This section is concerned with the measurement of thermal properties of OAEs in molecular junctions. Molecular wires such as OPEs have been proposed as candidate materials for low-cost, environmentally-friendly thermoelectric generators, ${ }^{27-29}$ i.e. devices that can harvest (waste) heat and transform it into electricity. An effective thermoelectric material should conduct electrons efficiently and heat poorly. Thermoelectric performance can be summarised using a 'thermoelectric figure of merit,' $Z T,{ }^{27-29}$ according to the equation:

$$
Z T=\frac{G S^{2} T}{\kappa_{\mathrm{p}}+\kappa_{\mathrm{e}}}
$$

A higher $Z T$ indicates more favourable performance. The electrical conductance, $G$, was discussed in detail in section 3 . $S$ is the Seebeck coefficient, or thermopower, of a material. $T$ is the temperature of the material (although as a thermoelectric device is reliant on a temperature gradient this term can prove problematic to define in practice). ${ }^{243}$ The terms $\kappa_{\mathrm{p}}$ and $\kappa_{\mathrm{e}}$ represent the thermal conductance due to phonons and electrons, respectively, and may be combined into a single $\kappa$ term representing total thermal conductance.

Experimentally, the Seebeck coefficient of a molecular wire is determined by measuring the thermoelectric voltage $(\Delta V)$ across a molecular junction with different temperature differentials $(\Delta T)$ between the electrodes. A plot of $\Delta V$ against $\Delta T$ should be linear with a gradient of $-S .{ }^{33,85}$ Transmission function calculations used in molecular conductance studies (Fig. 6) also provide information about the Seebeck coefficient. $S$ is proportional to the slope of $T(E)$, but has the opposite sign. When the HOMO is closest to $E_{\mathrm{F}}, T(E)$ has negative slope at $E_{\mathrm{F}}$ and $S$ is therefore positive. The opposite holds when the LUMO is closest to $E_{\mathrm{F} .}{ }^{85} \mathrm{DQI}$ antiresonances can result in large gradients of $T(E)$ in the HOMO-LUMO gap and have been identified as a potential means to increase $S .{ }^{244}$ However, as discussed in section 3.2, DQI results in reduced electronic conductance so the resulting increase in $S$ may not afford a concomitant increase in $Z T$. It has been proposed that by carefully controlling the position of a DQI feature, $S$ could be increased without significantly reducing $G ;^{34,244}$ note that the direction in which an antiresonance is shifted would affect the slope of $T(E)$ and hence the sign of $S$.

Surprisingly, given their ubiquity in charge transport studies, relatively few experimental studies have investigated the thermoelectric properties of OAEs, particularly with respect to structure-property relationships. Oligophenylenes are much more prevalent. ${ }^{29,30}$ Substituted benzene rings are therefore widely studied, including in the earliest report of Seebeck coefficients for single molecules from $2007 .^{85}$

\subsection{Influence of anchoring groups on Seebeck coefficient}

A key insight of early molecular thermopower studies is that the sign of the Seebeck coefficient is dependent on the anchoring group of the molecule. This is logical as the anchoring group determines the dominant orbital for conductance, and hence the slope of $T(E)$ at $E_{\mathrm{F} \cdot}{ }^{33}$ Early studies of functionalised benzenes showed that $S$ was positive for thiol ${ }^{33,85,203,245}$ and amine $^{245}$ anchoring groups but negative for nitrile anchoring groups. ${ }^{203}$ Subsequent studies of OAEs agree with these observations for thiols and amines, ${ }^{86,152}$ and additionally show that $S$ is negative for pyridyl, ${ }^{139,246}$ methylthio ${ }^{126,139,246}$ and $\mathrm{DHBT}^{202}$ anchoring groups. Interestingly, the structurally similar DMBT anchoring group was found to afford positive values of $S .{ }^{166}$ The electrode material has been shown to affect $S$; changing from Au to Ni electrodes in an STM-BJ study saw $S$ change from positive to negative for benzene-1,4-dithiol. This was attributed to spin hybridisation between the molecular orbitals and the Ni electrodes. ${ }^{247}$

Anchoring group effects in larger OAEs were investigated using c-AFM in a comparison of OAE3s with central 9,10-connected anthracene units $(76,77,74$ and $\boldsymbol{p}$-23, Fig. 38). These varied only in their anchoring groups, which were either both pyridyl (76), both methylthio (74), one of each (77), or both 


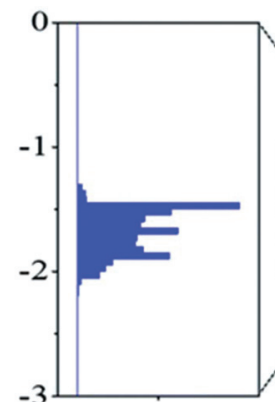

76

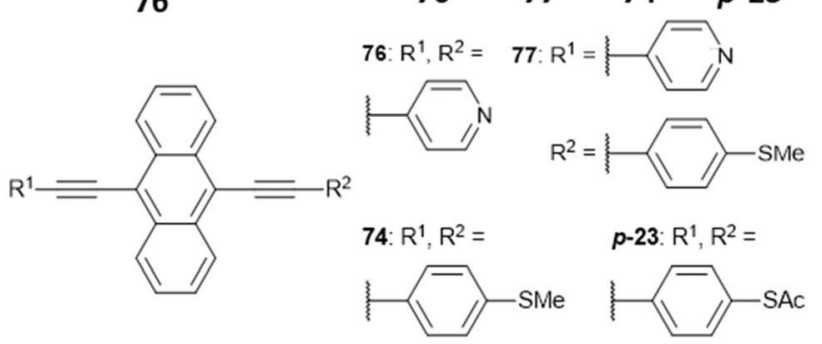

Fig. 38 Seebeck coefficient distributions from c-AFM studies (above) and structures (below) of OAE3 wires used by Ismael et al. to investigate anchoring group effects on thermopower in OAEs. This figure has been reproduced from ref. 139 with permission from The Royal Society of Chemistry, copyright 2020.

(acetyl protected) thiols $\left(\boldsymbol{p}\right.$-23). ${ }^{139}$ As shown in Fig. 38, the magnitude of $S$ was low for the bispyridyl species $76(-2.50 \mu \mathrm{V}$ $\mathrm{K}^{-1}$ ), increased by a factor of $c a .2$ when one pyridyl was replaced by para-(methylthio)phenyl to give 77 , and increased significantly to $-21.6 \mu \mathrm{V} \mathrm{K}^{-1}$ for $\mathbf{7 4}$, with two para-(methylthio) phenyl anchors. For thiol anchored $\boldsymbol{p}$-23 the sign of $S$ was positive, with an intermediate magnitude $\left(+11.0 \mu \mathrm{V} \mathrm{K}^{-1}\right)$ compared to analogues $\mathbf{7 6}$ and $\mathbf{7 4 .}{ }^{139}$ The tenfold increase in $S$ for $-\mathrm{SMe}$ $v s$. pyridyl anchors highlights the importance of anchor group selection in the design of OAEs for thermoelectric applications. The authors highlighted the relevance of strategies for structural control of $S$ in thin films to future device applications.

It should be noted that the structure-property relationships described above are not necessarily transferable to other systems. For example, for biphenylene-centred OAE3s the magnitude of $S$ was found to be larger with pyridyl anchoring groups $\left(-9.71 \pm 1.06 \mu \mathrm{V} \mathrm{K}^{-1}\right)$ than with $-\mathrm{SMe}$ anchoring groups $\left(-6.88 \pm 0.67 \mu \mathrm{V} \mathrm{K} \mathrm{K}^{-1}\right) .{ }^{246}$ However, in this case a DQI feature was observed in the transmission function of the pyridyl-anchored system, but not the -SMe anchored system. Clear scope remains for in-depth studies to better define all aspects of the relationship between anchoring groups, other structural features, electrode material, and the sign and magnitude of $S$.

\subsection{Seebeck coefficient: structure-property relationships}

Investigations into the effect of structural features of OAEs other than the anchoring group on $S$ are relatively rare. These predominantly recent works consider similar properties to comparable studies of electronic conductance: connectivity and QI, conjugation and aromaticity, and side groups.

Miao et al. determined $S$ for para- and meta-connected OPE3 dithiols in one of the first experimental studies to compare the effects of CQI and DQI on thermopower. ${ }^{152} S$ was higher for meta-connectivity when measured using both STM-BJ (meta: $+20.9 \pm 15.4 \mu \mathrm{V} \mathrm{K}{ }^{-1}$, para: $+10.8 \pm 9.5 \mu \mathrm{V} \mathrm{K}^{-1}$ ) and AFM (meta: $+22.5 \pm 1.1 \mu \mathrm{V} \mathrm{K}^{-1}$, para: $+8.0 \pm 0.8 \mu \mathrm{V} \mathrm{K}^{-1}$, Fig. 39a and b). ${ }^{152}$ These results agreed with expectations based on transmission function calculations, in which the DQI feature associated with meta-connectivity increased the slope of $T(E)$ around $E_{\mathrm{F}}$, and hence the magnitude of $S$, relative to para-connectivity (Fig. 39c). This crucial experimental confir-
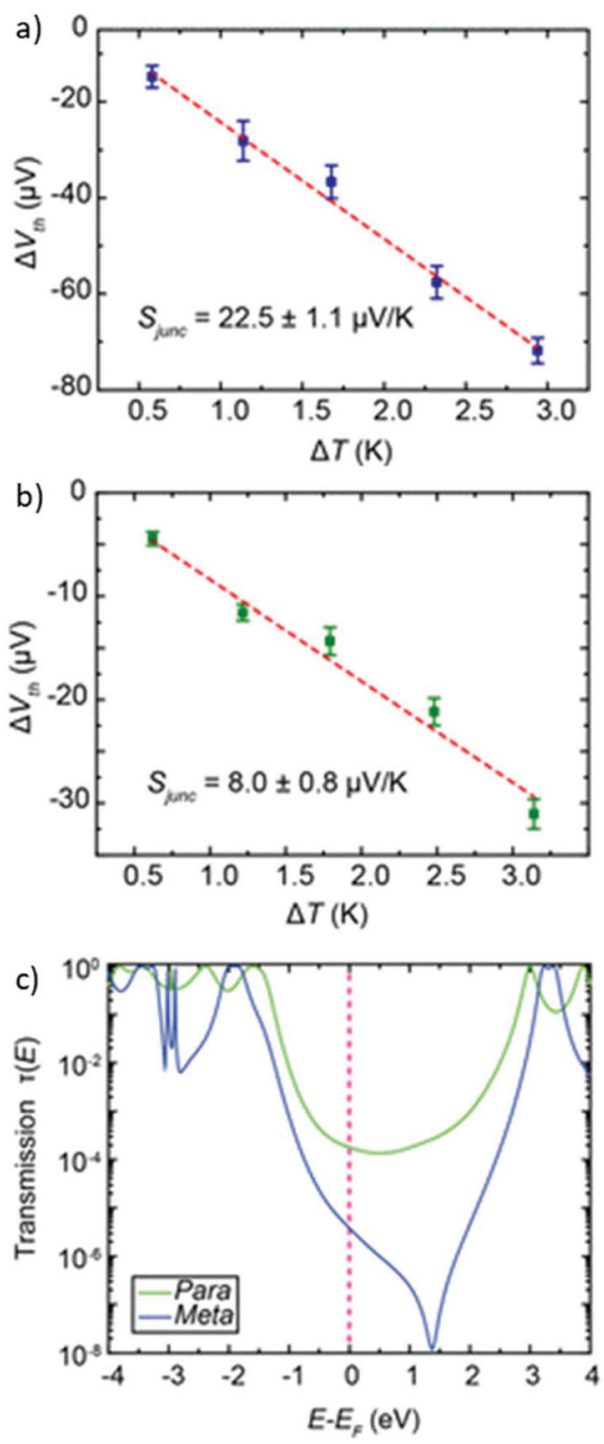

Fig. 39 Thermoelectric voltage as a function of $\Delta T$ for monolayers of meta-OPE3 dithiol (a) and para-OPE3 dithiol (b) determined using an AFM method (N.B. the gradient of these plots is equal to $-S$ ), and calculated transmission functions for single-molecule junctions containing the same molecules (c). This figure has been adapted from ref. 152 with permission from the American Chemical Society, copyright 2018. 
mation of theoretical predictions that QI could be used to manipulate thermoelectric properties prompted many subsequent studies of structure-property relationships.

Wang et al. compared two different conductance pathways for which CQI occurs using differently connected anthracenecentred OAE3s with thiol and methylthio anchors (56 and 57 in Fig. 28). ${ }^{126}$ As discussed in section 3.2, regardless of the anchoring group, electronic conductance was higher for 9,10(56, red) than 1,5 -connectivity $(57$, blue). In contrast, the effect of connectivity on $S$ depended on the anchoring group. For -SMe anchoring (negative $S$ ), the magnitude of $S$ was larger for the less conductive isomer $57\left(-31.8 \mu \mathrm{V} \mathrm{K} \mathrm{K}^{-1}\right.$ for 1,5- vs. $-23.4 \mu \mathrm{V} \mathrm{K}^{-1}$ for 9,10-connectivity) whereas for thiol anchoring (positive $S$ ) the more conductive isomer $\mathbf{5 6}$ also had higher $S$ $\left(+10.4 \mu \mathrm{V} \mathrm{K}^{-1}\right.$ for $1,5-v s .+12.1 \mu \mathrm{V} \mathrm{K}{ }^{-1}$ for 9,10 -connectivity). ${ }^{126}$ Analysis of the transmission functions emphasised the sensitivity of $S$ to molecular orbital energies and the need to correctly determine $E_{\mathrm{F}}$ in theoretical studies. While it is clear that CQI can be used to enhance $S$, the concomitant effects of anchoring groups are likely to make design of such systems more challenging than those based on DQI.

A comparison of methylthio-anchored OAE3s with central 1,4-connected biphenylene, naphthalene and anthracene units (69-71, respectively, Fig. 36) explored the effects of antiaromaticity and conjugation on $S .{ }^{246}$ Calculations showed that the energy of the LUMO was similar for all three systems, but that the HOMO-LUMO gap decreased in size as the central unit was varied from biphenylene to naphthalene to anthracene. The slope of $T(E)$ at (an empirically corrected) $E_{\mathrm{F}}$ suggested that the magnitude of $S$ should follow the trend $69>70>71$, as the centre of the HOMO-LUMO gap lies closer to (the corrected) $E_{\mathrm{F}}$ as conjugation increases. Experimentally the magnitude of $S$ followed the trend $69\left(-6.88 \pm 1.06 \mu \mathrm{V} \mathrm{K}^{-1}\right)>71$ $\left(-4.13 \pm 1.52 \mu \mathrm{V} \mathrm{K}^{-1}\right)>70\left(-1.62 \pm 0.21 \mu \mathrm{V} \mathrm{K}{ }^{-1}\right)$, which can be corrected for computationally by adjusting the energy levels. ${ }^{246}$ There was no clear relationship between $S$ and conjugation, the energetics of the system appeared to be more influential.

Side group functionalisation was shown to have a small effect on the thermopower of benzene-1,4-dithiol derivatives in 2008. ${ }^{203}$ Similarly limited variation of $S$ was observed recently by Dekkiche et al. in a comparison of DHBT-anchored OAE3s in which the central aryl unit was varied between benzene, 2,5-dimethoxybenzene and 9,10-connected anthracene. ${ }^{202}$ In this series, $S$ was largest for the benzene species $\left(-11.4 \pm 0.5 \mu \mathrm{V} \mathrm{K}{ }^{-1}\right)$, followed by the anthracene $(-8.7 \pm 1.6 \mu \mathrm{V}$ $\left.\mathrm{K}^{-1}\right)$ then the dimethoxybenzene derivative $\left(-7.9 \pm 1.3 \mu \mathrm{V} \mathrm{K} \mathrm{K}^{-1}\right)$. This trend could not be easily rationalised using transmission functions; the conductance is LUMO dominated but calculations could not accurately reproduce the subtle differences within the series. ${ }^{202}$ As for the above study where conjugation was varied, ${ }^{246}$ precise energetics appear to be more influential than structural effects. An interesting and unexpected observation was that the anthracene-centred wire displayed enhanced thermal stability compared to the rest of the series, allowing data to be collected for a larger range of $\Delta T$ values. $^{202}$
The thermopower of DMBT-anchored OPEs was recently compared to related metal bis(acetylide) complexes. $S$ increased by up to $c a$. $50 \%$ upon replacement of the central benzene ring of an OPE3 with a Ru or Pd centre, although in all cases the measured values were modest, with a maximum of $+7.1 \mu \mathrm{V} \mathrm{K}^{-1}$ for the Ru complex. ${ }^{166}$

Based on these early studies, it remains to be seen whether clear structure-property relationships relating to $S$ will be established to the same extent as those relating to $G$ discussed in section 3. It remains a challenge to separate the effects of different structural factors from both one another and influences such as the energetics of the junction. This is further complicated by the often small variation in $S$ for structurally similar systems.

\subsection{Thermal conductance}

Theoretical studies of OPEs have shown that structural modifications influence $\kappa_{\mathrm{p}}$, which is the dominant contributor to thermal conductance at room temperature. ${ }^{248}$ metaConnectivity afforded lower $\kappa_{\mathrm{p}}$ than para-connectivity due to phonon interference effects; this effect was more pronounced for OPE3 systems than for OPE1 species (i.e. single benzene rings). ${ }^{248,249}$ Calculations have also shown that replacing a proton with a halogen substituent reduced $\kappa_{\mathrm{p}}$ for OPE3 and OPE1 species, with a larger reduction in $\kappa_{\mathrm{p}}$ as the mass of the halogen increases. ${ }^{248}$

Experimental determination of $\kappa$ for molecular junctions is challenging. ${ }^{250}$ Approaches based on laser spectroscopy and scanning thermal microscopy exist, but have primarily been applied to alkanethiols. ${ }^{250}$ However, one of the first studies of single-molecule thermal conductance compared OPE3 dithiol with 1,8-octanedithiol. ${ }^{89}$ This ground-breaking work utilised a specially designed suspended micro-electro-mechanical system (MEMS) as a substrate for molecular assembly in conjunction with a custom-built STM-BJ setup (Fig. 40a). The thermal conductance of OPE3 dithiol $\left(20 \pm 6\right.$ pW K ${ }^{-1}$, Fig. 40b) was lower than that of octanedithiol $\left(29 \pm 8 \mathrm{pW} \mathrm{K}{ }^{-1}\right.$, Fig. $\left.40 \mathrm{c}\right) .{ }^{89}$ The authors note that this variation is fairly small, and highlight the potential of phonon engineering strategies, such as phonon interference, ${ }^{251}$ as a means to minimise $\kappa$ and to increase $Z T$. Structure-property relationships within series of OAEs have promise as a means to increase understanding of phonon engineering, provided that the capability to carry out single-molecule thermal conductance studies becomes more widespread.

A key consequence of the recent development of methods to experimentally determine $\kappa$ is that it is now possible to measure each of the values that contribute to the thermoelectric figure of merit $Z T$ (i.e. $G, S, T$ and $\kappa$ ). This is of fundamental importance to the characterisation of materials for thermoelectric applications, for which optimisation of $Z T$ is required in order to achieve viable devices.

\subsection{Outlook}

Most studies of the thermoelectric properties of OAEs are from the last few years. Moving forward OAEs have the potential to 
a)

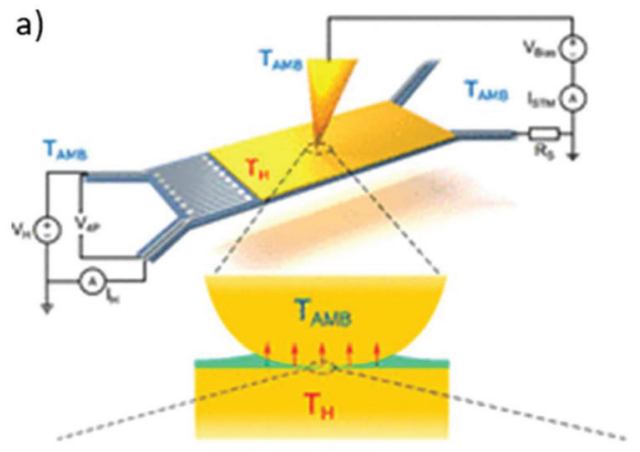

b)

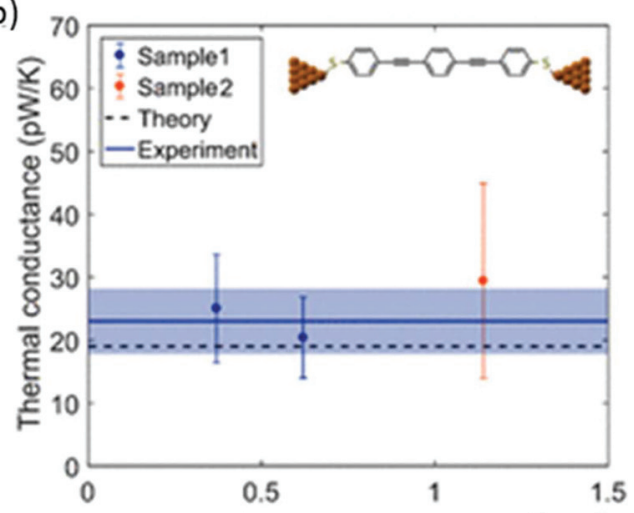

Slope of thermal background $\left(n W K^{1} \mathrm{~nm}^{-1}\right)$

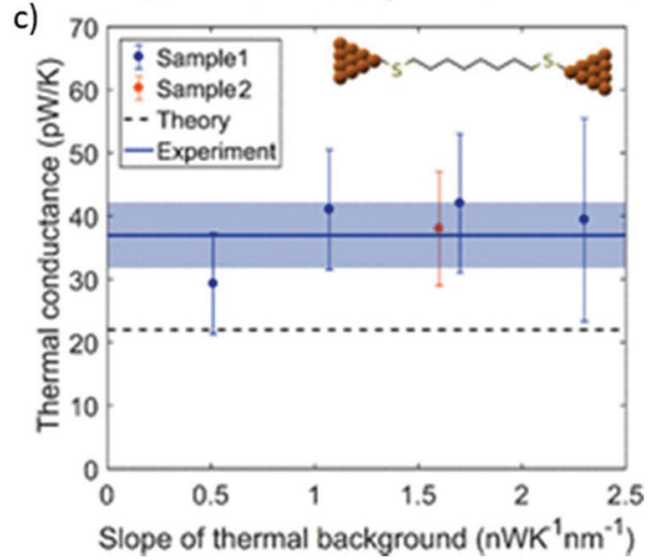

Fig. 40 (a) Illustration of the MEMS/STM-BJ setup used by Mosso et al. to determine single molecule thermal conductance; (b) data used to determine the single molecule thermal conductance of OPE3 dithiol; (c) data used to determine the single molecule thermal conductance of octanedithiol. This figure has been reproduced from ref. 89 with permission from the American Chemical Society, copyright 2019.

become the workhorse molecules of molecular thermoelectric research, paralleling their role in molecular electronics. It is reasonable to anticipate studies seeking a clearer understanding of structural effects on $S$ and $\kappa$ in order to optimise $Z T$. The wide structural variation possible with OAEs provides clear opportunities in phonon engineering, particularly as experimental measurements of thermal conductance become more accessible.

\section{Recent developments and future prospects}

The previous section anticipated growing interest in the thermoelectric properties of OAEs in the near future. This section will consider some other recent developments that are likely to influence future studies of OAEs in junctions.

\subsection{Data analysis}

In recent years considerable efforts have been made to apply machine learning to the analysis of molecular conductance traces. In early single-molecule conductance studies manual selection of conductance traces was not uncommon, ${ }^{24}$ despite being time-consuming and carrying a risk of bias. ${ }^{167}$ The development of data sorting methods such as clustering algorithms provides an opportunity to analyse data objectively and gain even more detailed insight into the behaviour of molecules in junctions. Many of these methods were pioneered using datasets collected for OAE derivatives. ${ }^{69,146,167,178,252-256}$

The utility of these techniques is exemplified by a study of porphyrin-centred OAE3s by El Abbassi et al., who used a clustering algorithm in conjunction with a range of model systems to assign classes of conductance trace to different junction geometries (Fig. 41). ${ }^{146}$ Note the identification of unconventional anchoring behaviour assigned to the less conductive classes B and C. The model systems each contained different features of the molecule shown in Fig. 41. By applying the clustering algorithm to conductance data for each model system, classes could be associated with specific structural features and the junction geometry deduced. This emphasises the importance of interdisciplinary collaboration in molecular electronics: additional synthesis greatly enhanced the outcome of the data analysis. The proposal of novel conductance pathways also lends itself to associated computational work, as these junction geometries can be modelled. Automated data analysis methods of this type will likely become commonplace in single-molecule conductance studies. They permit analysis of highly functionalised molecular wires that are likely to afford complex conductance traces.

\subsection{Junction materials and methods}

Historically, molecular junctions have relied on metallic electrodes, with single-molecule studies particularly reliant on gold electrodes. The above discussion has already made clear the growing interest in alternative junction materials and designs, including nickel electrodes in STM-BJ studies, ${ }^{247}$ ITO-molecule-Au junctions ${ }^{165}$ and bridging nanogaps between $\mathrm{CNT}^{76,77}$ or graphene $e^{73-75}$ electrodes with molecules. Graphene-based junctions are of particular interest owing to the notable properties of graphene and its compatibility with organic materials. ${ }^{257}$ Recently, two graphene-based MCBJ approaches have been reported. The first is based directly on the breaking of a graphene sheet suspended between gold electrodes, ${ }^{258}$ but to our knowledge has not yet been applied to the study of molecular junctions. A second approach with graphene-coated 
a)

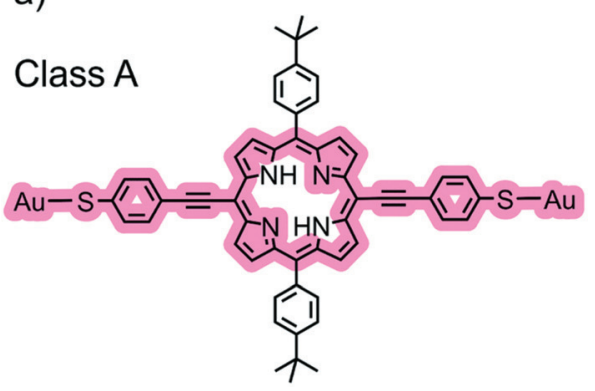

b)

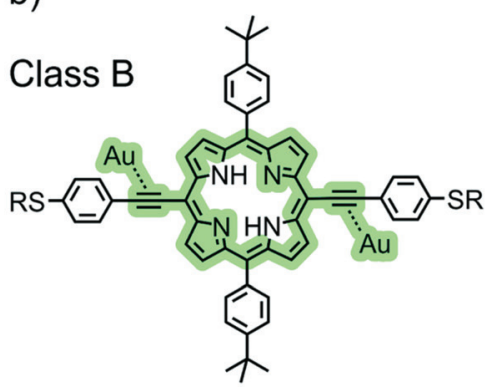

c)

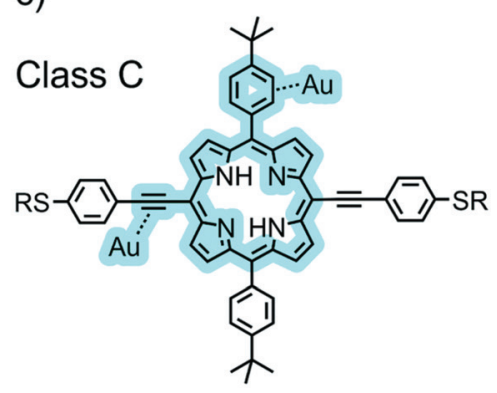

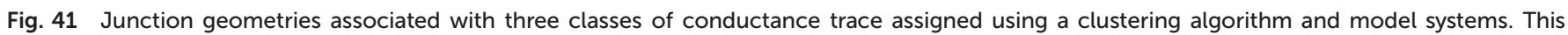
figure has been reproduced from ref. 146 with permission from The Royal Society of Chemistry, copyright 2019.

copper wires as electrodes has been used to measure the conductance of various fullerenes ${ }^{259}$ and the cross-plane transport of a series of polycyclic aromatic hydrocarbons. ${ }^{260}$

The development of new approaches and innovations for junctions with metal electrodes also continues. One recent example is based on the closure of nanogaps pre-coated with molecules using electrical field migration. A nanogap pretreated with 1,4-benzenedithiol was subjected to $I-V$ sweeps and repeatedly exhibited a conductance corresponding to that observed for the same molecule in an MCBJ. ${ }^{261}$ Another innovative development is the use of an external electric field to influence junction formation probability in STM-BJ experiments. ${ }^{262}$ It was found that OPEs with methylthio anchoring groups were increasingly likely to form junctions as the bias was increased, whereas thiol, amine and pyridyl anchors all showed reduced junction formation probability with increasing bias. This meant it was possible to demonstrate selective, preferential junction formation in a mixture of methylthioand thiol-anchored OPEs based on the applied electric field. ${ }^{262}$

OAE derivatives are outstanding candidate molecules for developing novel molecular junction methodologies and as benchmark compounds for investigations of modifications to existing techniques. The ease with which structural modification can be achieved facilitates the translation of OAE wires to new electrode surfaces that require alternative anchoring groups.

\subsection{Molecular spintronics}

In spintronics, electrons serve as more than simple charge carriers; their spin is also important, and can be used to create logic or memory devices. ${ }^{263}$ The idea of designing molecular wires with spintronic applications is longstanding, ${ }^{264,265}$ but experimental studies are an area of relatively recent and increasing growth. Radical functionalised OPE-like materials were identified as candidate spin-filters in a computational study in 2010. ${ }^{265}$ The proposed materials did not, however, have structures associated with stable radicals. A study of an OPE3 with a side group bearing a stable (2,2,6,6-tetramethylpiperidin-1-yl)oxyl (TEMPO) radical (78, Fig. 42) determined that this system, in which the radical is not conjugated to the backbone, was not a promising spin filter. ${ }^{266}$ However, experimental studies showed that $\mathbf{7 8}$ displayed unprecedentedly

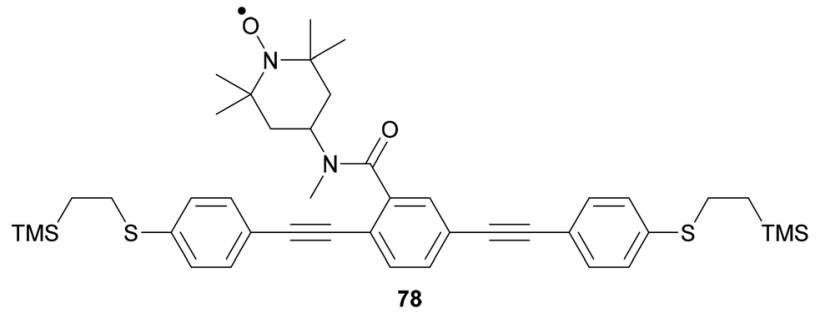

Fig. 42 OPE3 78, with a side group bearing a TEMPO radical functionality.

large magnetoresistance, much higher than that of an unfunctionalised OPE3. ${ }^{266}$

The limited spin filtering behaviour of $\mathbf{7 8}$ is supported by a recent computational study of other structurally similar radical-bearing OPE3s, which found that good orbital overlap between the $\pi$-systems of the OPE backbone and radical is needed to achieve appreciable spin filtering. ${ }^{267}$ Unfortunately this is difficult to achieve experimentally as significant steric shielding, which promotes divergence from coplanarity, is usually required to stabilise organic radicals. It was noted that larger spin polarisation (and potentially enhanced Kondo features, see below) might be achieved by using meta-OPE analogues, where reduced intramolecular steric hindrance would be expected. $^{267}$ This would lead to a more coplanar backbone and hence increased spin delocalisation along the OPE wire, although as discussed in section 3.2 lower overall conductance would be expected. Incorporating radical functionality directly into the molecular backbone may not be straightforward either; a (nonOAE) molecular wire with an otherwise stable Blatter radical in its backbone appeared to oxidise to a closed-shell species when bound between electrodes in STM-BJ experiments. ${ }^{268}$

An alternative approach to molecular spintronics is the use of junctions based on ferromagnetic electrodes, which have a different density of states for spin-up and spin-down electrons. Hybridisation upon binding with a molecule creates spin polarised molecular orbitals, and therefore the resulting molecular junctions have potential as spin filters. ${ }^{269}$ The combination of nickel electrodes and a series of OAE3 wires has been investigated computationally. ${ }^{270}$ By considering meta-OPE3 and analo- 
gous meta-OAE3s substituted with heteroatoms (both within the backbone and as pendent substituents) it was found that the location of a DQI feature affects not only the magnitude of conductance, as discussed in section 3.4, but also the extent of spin polarisation. The least conductive species gave the highest spin polarisation. However, through appropriate heteroatom substitution relatively high spin selectivity was achieved alongside appreciable molecular conductance. ${ }^{270}$ OAE wires designed for QI studies should therefore have experimental utility in spintronics in the near-future. Ni break junctions have already been developed, ${ }^{247,271}$ and methods to experimentally analyse spin filtering in molecular junctions have been demonstrated. ${ }^{272}$ Combining these techniques with appropriate molecules, such as OAEs, should allow detailed interdisciplinary studies of molecular spintronics. As a general rule low-spin and high-spin states are stable at low and high temperatures, respectively, and low temperature vacuum conditions are required to achieve a stable reversible spin switch. ${ }^{273}$ Therefore, it is an interesting challenge to raise operating temperatures to room temperature.

The Kondo effect, which is associated with unpaired spins, is another spin phenomenon that is of interest in molecular junction studies, ${ }^{19,274}$ with potential applications in switchable devices. The Kondo effect has been observed in junctions containing molecules bearing an unpaired electron and derives from interactions between electrons including the Pauli exclu- sion principle (see ref. 274 for a more detailed overview, which is beyond the scope of the present discussion). A typical manifestation in molecular junctions containing a spin- $\frac{1}{2}$ feature is the presence of a distinct zero-bias conductance below a critical Kondo temperature, $T_{\mathrm{K}}$. Cryogenic conditions are required to access $T_{\mathrm{K}}$, which for single-molecule junctions is typically on the order of tens of Kelvin or below. ${ }^{211,274,275}$ There are relatively few examples of OAE wires that display the Kondo effect as many studies have selected alternative backbones to more easily incorporate unpaired spins in the form of e.g. triphenylmethane radicals ${ }^{276}$ or metal complexes. ${ }^{277,278}$

The OPE5-exTTF cruciform molecule 79 (Fig. 43a, top) was studied experimentally in a three-terminal device to probe electrical control of organic magnetism. ${ }^{211}$ The molecule was reversibly switched between three redox states and the resulting radicals were found to couple antiferromagnetically or ferromagnetically depending on the relative locations of the charges on the molecular backbone. Differing Kondo features and zero-bias conductances were observed for the various charged states due to the differing number of interacting spins (Fig. 43b-d). ${ }^{211}$ The system was described as acting like a twoqubit gate, indicating the potential of Kondo effect molecules in quantum computing applications.

Kondo-like behaviour was reported for the dinuclear $\mathrm{Co}$ (II) complex 80 (Fig. 43a, bottom) in which the two metal ions
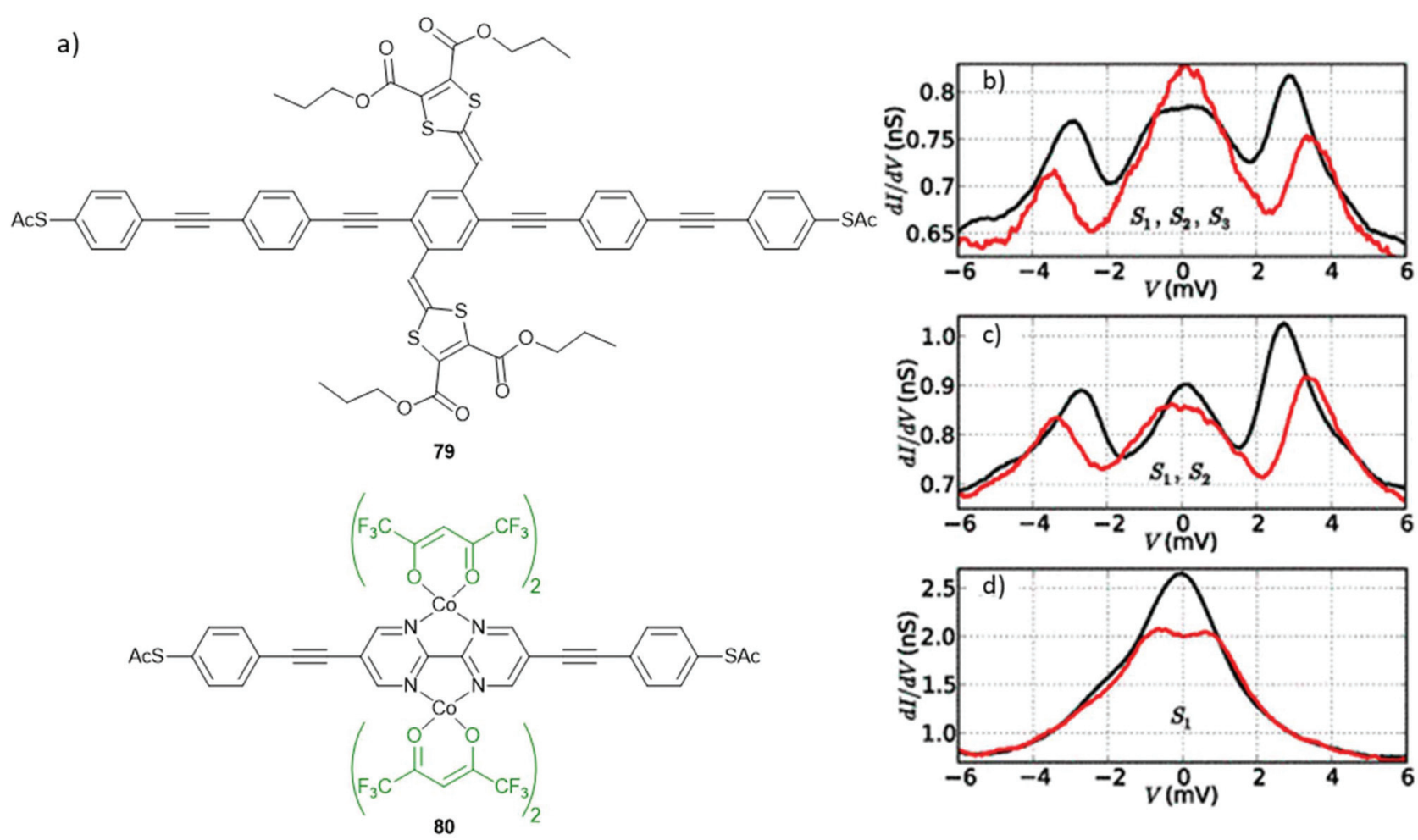

Fig. 43 (a) Structures of OAE wires used in studies investigating the Kondo effect; (b-d) Differential conductance versus voltage for a junction containing (deprotected) molecule 79 averaged over different gating voltage ranges [(b): 0.2-0.4 V, (c) 1.4-1.5 V, (d) 2.4-2.5 V] with either zero (black lines) or $8 \mathrm{~T}$ (red lines) applied magnetic field. The $S_{X}$ notation $(X=1-3)$ indicates the spin states interacting in each bias range. In each case, the non-zero conductance at zero-bias is indicative of the Kondo effect. Panels $(b-d)$ have been reproduced from ref. 211 with permission from the American Physical Society, copyright 2012. 
(bearing ancillary ligands) are each bound to the same OAE3 wire with a central ligating bipyrimidine unit. ${ }^{275}$ Switching behaviour based on applied bias was assigned to the existence of pseudosinglet and pseudo-triplet states. The latter state is magnetically active and exhibited a zero-bias conductance feature that could be split by an applied magnetic field, as seen for Kondo features in other studies. ${ }^{275}$ In contrast, the pseudo-singlet state and the free ligand did not display Kondo-like properties.

Behaviour resembling the Kondo effect was observed for TEMPO-functionalised wire 78 (Fig. 42). However, the authors of the study do not definitively assign the observed zero-bias conductance to the Kondo effect, noting that additional measurements would be required to confirm this and that the existing data would require an unusually large $T_{\mathrm{K}}$ of $c a$. $360 \mathrm{~K}^{266}$ There remains much to explore regarding the Kondo effect in molecular junctions, and appropriately functionalised OAE wires provide a convenient means to better understand and exploit this phenomenon.

\section{Conclusions}

The OAE framework provides a versatile and widely-used basis for molecular wire design. Through skilled synthetic chemistry the length, connectivity, aryl units, anchoring groups and side chain functionality of OAEs can all be systematically varied. This has enabled the design and preparation of a vast array of molecules with targeted properties that have been used to investigate and optimise the electronic or thermal properties of molecular wires. This unprecedented synthetic flexibility means that OAEs will almost certainly continue to be the workhorses of molecular electronics, for example in experimental and computational studies of QI behaviour. Their extensive prior study makes OAEs ideal materials to benchmark junctions based on new materials and methods. OAEs are expected to find increasing use in the expanding fields of molecular thermoelectrics and spintronics.

\section{Author contributions}

Both co-authors jointly planned the outline and scope of the article. L. J. O. initiated the writing and wrote the majority of the text, with input from M. R. B. Funding to support the work was obtained by M. R. B.

\section{Conflicts of interest}

There are no conflicts to declare.

\section{Acknowledgements}

This work was supported by EPSRC grant EP/P027520/1 and EC H2020 FET Open projects: grant agreement numbers 767187 'QuIET' and 766853 'EFINED'.

\section{Notes and references}

1 D. G. Whitten, Y. Tang, Z. Zhou, J. Yang, Y. Wang, E. H. Hill, H. C. Pappas, P. L. Donabedian and E. Y. Chi, Langmuir, 2019, 35, 307-325.

2 F. A. Monge, P. Jagadesan, V. Bondu, P. L. Donabedian, L. Ista, E. Y. Chi, K. S. Schanze, D. G. Whitten and A. M. Kell, ACS Appl. Mater. Interfaces, 2020, 12, 5568855695.

3 M. K. Smith and O. Š. Miljanić, Org. Biomol. Chem., 2015, 13, 7841-7845.

4 C. Schweez, P. Shushkov, S. Grimme and S. Höger, Angew. Chem., Int. Ed., 2016, 55, 3328-3333.

5 C. Rest, M. J. Mayoral, K. Fucke, J. Schellheimer, V. Stepanenko and G. Fernández, Angew. Chem., Int. Ed., 2014, 53, 700-705.

6 A. Krywko-Cendrowska, D. Szweda and R. Szweda, Processes, 2020, 8, 539.

7 M. Hergert, M. Bender, K. Seehafer and U. H. F. Bunz, Chem. - Eur. J., 2018, 24, 3132-3135.

8 H. Yang, Y. Du, S. Wan, G. D. Trahan, Y. Jin and W. Zhang, Chem. Sci., 2015, 6, 4049-4053.

9 F. Silvestri and A. Marrocchi, Int. J. Mol. Sci., 2010, 11, 1471-1508.

10 X. Zeng, R. Kieffer, B. Glettner, C. Nürnberger, F. Liu, K. Pelz, M. Prehm, U. Baumeister, H. Hahn, H. Lang, G. A. Gehring, C. H. M. Weber, J. K. Hobbs, C. Tschierske and G. Ungar, Science, 2011, 331, 1302-1306.

11 D. Lehnherr, C. Chen, Z. Pedramrazi, C. R. DeBlase, J. M. Alzola, I. Keresztes, E. B. Lobkovsky, M. F. Crommie and W. R. Dichtel, Chem. Sci., 2016, 7, 6357-6364.

12 M. Rickhaus, A. Vargas Jentzsch, L. Tejerina, I. Grübner, M. Jirasek, T. D. W. Claridge and H. L. Anderson, J. Am. Chem. Soc., 2017, 139, 16502-16505.

13 S. H. Chanteau and J. M. Tour, J. Org. Chem., 2003, 68, 8750-8766.

14 B. Xu and N. J. Tao, Science, 2003, 301, 1221-1223.

15 M. A. Reed, C. Zhou, C. J. Muller, T. P. Burgin and J. M. Tour, Science, 1997, 278, 252-254.

16 D. J. Wold and C. D. Frisbie, J. Am. Chem. Soc., 2000, 122, 2970-2971.

17 R. C. Chiechi, E. A. Weiss, M. D. Dickey and G. M. Whitesides, Angew. Chem., Int. Ed., 2008, 47, 142144.

18 D. Xiang, X. Wang, C. Jia, T. Lee and X. Guo, Chem. Rev., 2016, 116, 4318-4440.

19 P. Gehring, J. M. Thijssen and H. S. J. van der Zant, Nat. Rev. Phys., 2019, 1, 381-396.

20 D. Vuillaume, ChemPlusChem, 2019, 84, 1215-1221.

21 Y. Zhang, Z. Zhao, D. Fracasso and R. C. Chiechi, Isr. J. Chem., 2014, 54, 513-533.

22 G. Wang, T.-W. Kim and T. Lee, J. Mater. Chem., 2011, 21, 18117-18136.

23 R. M. Metzger, Chem. Rev., 2015, 115, 5056-5115.

24 C. Huang, A. V. Rudnev, W. Hong and T. Wandlowski, Chem. Soc. Rev., 2015, 44, 889-901. 
25 F. Evers, R. Korytár, S. Tewari and J. M. van Ruitenbeek, Rev. Mod. Phys., 2020, 92, 035001.

26 L. Herrer, S. Martín and P. Cea, Appl. Sci., 2020, 10, 6064.

27 L. Cui, R. Miao, C. Jiang, E. Meyhofer and P. Reddy, J. Chem. Phys., 2017, 146, 092201.

28 K. Wang, E. Meyhofer and P. Reddy, Adv. Funct. Mater., 2020, 30, 1904534.

29 L. Rincón-García, C. Evangeli, G. Rubio-Bollinger and N. Agraït, Chem. Soc. Rev., 2016, 45, 4285-4306.

30 S. Park, H. Kang and H. J. Yoon, J. Mater. Chem. A, 2019, 7, 14419-14446.

31 C. J. Lambert, Chem. Soc. Rev., 2015, 44, 875-888.

32 H. Sadeghi, Nanotechnology, 2018, 29, 373001.

33 A. Tan, J. Balachandran, S. Sadat, V. Gavini, B. D. Dunietz, S.-Y. Jang and P. Reddy, J. Am. Chem. Soc., 2011, 133, 8838-8841.

34 L. J. O'Driscoll and M. R. Bryce, Nanoscale, 2021, 13, 1103-1123.

35 M. H. Garner, G. C. Solomon and M. Strange, J. Phys. Chem. C, 2016, 120, 9097-9103.

36 L. A. Zotti and E. Leary, Phys. Chem. Chem. Phys., 2020, 22, 5638-5646.

37 J. S. Schumm, D. L. Pearson and J. M. Tour, Angew. Chem., Int. Ed. Engl., 1994, 33, 1360-1363.

38 R. Wu, J. S. Schumm, D. L. Pearson and J. M. Tour, J. Org. Chem., 1996, 61, 6906-6921.

39 J. M. Tour, L. Jones, D. L. Pearson, J. J. S. Lamba, T. P. Burgin, G. M. Whitesides, D. L. Allara, A. N. Parikh and S. Atre, J. Am. Chem. Soc., 1995, 117, 9529-9534.

40 J. M. Tour, Acc. Chem. Res., 2000, 33, 791-804.

41 L. Jones, J. S. Schumm and J. M. Tour, J. Org. Chem., 1997, 62, 1388-1410.

42 D. L. Pearson and J. M. Tour, J. Org. Chem., 1997, 62, 1376-1387.

43 S. Huang and J. M. Tour, J. Org. Chem., 1999, 64, 8898-8906. 44 L. A. Bumm, J. J. Arnold, M. T. Cygan, T. D. Dunbar, T. P. Burgin, L. Jones, D. L. Allara, J. M. Tour and P. S. Weiss, Science, 1996, 271, 1705-1707.

45 J. Chen, M. A. Reed, C. L. Asplund, A. M. Cassell, M. L. Myrick, A. M. Rawlett, J. M. Tour and P. G. V. Patten, Appl. Phys. Lett., 1999, 75, 624-626.

46 T. A. Su, M. Neupane, M. L. Steigerwald, L. Venkataraman and C. Nuckolls, Nat. Rev. Mater., 2016, 1, 16002.

47 J. Reichert, R. Ochs, D. Beckmann, H. B. Weber, M. Mayor and H. v. Löhneysen, Phys. Rev. Lett., 2002, 88, 176804.

48 X. Xiao, B. Xu and N. J. Tao, Nano Lett., 2004, 4, 267-271.

49 X. Xiao, L. A. Nagahara, A. M. Rawlett and N. Tao, J. Am. Chem. Soc., 2005, 127, 9235-9240.

50 W. Haiss, H. van Zalinge, S. J. Higgins, D. Bethell, H. Höbenreich, D. J. Schiffrin and R. J. Nichols, J. Am. Chem. Soc., 2003, 125, 15294-15295.

51 W. Haiss, R. J. Nichols, H. van Zalinge, S. J. Higgins, D. Bethell and D. J. Schiffrin, Phys. Chem. Chem. Phys., 2004, 6, 4330-4337.

52 R. J. Nichols, W. Haiss, S. J. Higgins, E. Leary, S. Martin and D. Bethell, Phys. Chem. Chem. Phys., 2010, 12, 2801-2815.
53 M. P. Murrell, M. E. Welland, S. J. O'Shea, T. M. H. Wong, J. R. Barnes, A. W. McKinnon, M. Heyns and S. Verhaverbeke, Appl. Phys. Lett., 1993, 62, 786-788.

54 D. J. Wold, R. Haag, M. A. Rampi and C. D. Frisbie, J. Phys. Chem. B, 2002, 106, 2813-2816.

55 A. M. Rawlett, T. J. Hopson, L. A. Nagahara, R. K. Tsui, G. K. Ramachandran and S. M. Lindsay, Appl. Phys. Lett., 2002, 81, 3043-3045.

56 Z. Wei, T. Hansen, M. Santella, X. Wang, C. R. Parker, X. Jiang, T. Li, M. Glyvradal, K. Jennum, E. Glibstrup, N. Bovet, X. Wang, W. Hu, G. C. Solomon, M. B. Nielsen, X. Qiu, T. Bjørnholm, K. Nørgaard and B. W. Laursen, Adv. Funct. Mater., 2015, 25, 1700-1708.

57 T. P. Weihs, Z. Nawaz, S. P. Jarvis and J. B. Pethica, Appl. Phys. Lett., 1991, 59, 3536-3538.

58 N. A. Burnham, R. J. Colton and H. M. Pollock, Phys. Rev. Lett., 1993, 70, 247-247.

59 E. Gomar-Nadal, G. K. Ramachandran, F. Chen, T. Burgin, C. Rovira, D. B. Amabilino and S. M. Lindsay, J. Phys. Chem. B, 2004, 108, 7213-7218.

60 L. J. O’Driscoll, X. Wang, M. Jay, A. S. Batsanov, H. Sadeghi, C. J. Lambert, B. J. Robinson and M. R. Bryce, Angew. Chem., Int. Ed., 2020, 59, 882-889.

61 C. J. Querebillo, A. Terfort, D. L. Allara and M. Zharnikov, J. Phys. Chem. C, 2013, 117, 25556-25561.

62 M. Carlotti, M. Degen, Y. Zhang and R. C. Chiechi, J. Phys. Chem. C, 2016, 120, 20437-20445.

63 M. Carlotti, S. Soni, S. Kumar, Y. Ai, E. Sauter, M. Zharnikov and R. C. Chiechi, Angew. Chem., Int. Ed., 2018, 57, 15681-15685.

64 F. C. Simeone, H. J. Yoon, M. M. Thuo, J. R. Barber, B. Smith and G. M. Whitesides, J. Am. Chem. Soc., 2013, 135, 18131-18144.

65 D. Fracasso, H. Valkenier, J. C. Hummelen, G. C. Solomon and R. C. Chiechi, J. Am. Chem. Soc., 2011, 133, 9556-9563.

66 Y. Zhang, G. Ye, S. Soni, X. Qiu, T. L. Krijger, H. T. Jonkman, M. Carlotti, E. Sauter, M. Zharnikov and R. C. Chiechi, Chem. Sci., 2018, 9, 4414-4423.

67 M. Carlotti, S. Soni, X. Qiu, E. Sauter, M. Zharnikov and R. C. Chiechi, Nanoscale Adv., 2019, 1, 2018-2028.

68 S. Soni, G. Ye, J. Zheng, Y. Zhang, A. Asyuda, M. Zharnikov, W. Hong and R. C. Chiechi, Angew. Chem., Int. Ed., 2020, 59, 14308-14312.

69 Y. Liu, L. Ornago, M. Carlotti, Y. Ai, M. El Abbassi, S. Soni, A. Asyuda, M. Zharnikov, H. S. J. van der Zant and R. C. Chiechi, J. Phys. Chem. C, 2020, 124, 2277622783.

70 A. S. Blum, J. G. Kushmerick, S. K. Pollack, J. C. Yang, M. Moore, J. Naciri, R. Shashidhar and B. R. Ratna, J. Phys. Chem. B, 2004, 108, 18124-18128.

71 C. Ouyang, K. Hashimoto, H. Tsuji, E. Nakamura and Y. Majima, ACS Omega, 2018, 3, 5125-5130.

72 G. J. Ashwell, L. J. Phillips, B. J. Robinson, B. UrasinskaWojcik, C. J. Lambert, I. M. Grace, M. R. Bryce, R. Jitchati, M. Tavasli, T. I. Cox, I. C. Sage, R. P. Tuffin and S. Ray, ACS Nano, 2010, 4, 7401-7406. 
73 B. Limburg, J. O. Thomas, G. Holloway, H. Sadeghi, S. Sangtarash, I. C.-Y. Hou, J. Cremers, A. Narita, K. Müllen, C. J. Lambert, G. A. D. Briggs, J. A. Mol and H. L. Anderson, Adv. Funct. Mater., 2018, 28, 1803629.

74 J. O. Thomas, B. Limburg, J. K. Sowa, K. Willick, J. Baugh, G. A. D. Briggs, E. M. Gauger, H. L. Anderson and J. A. Mol, Nat. Commun., 2019, 10, 4628.

75 B. Limburg, J. O. Thomas, J. K. Sowa, K. Willick, J. Baugh, E. M. Gauger, G. A. D. Briggs, J. A. Mol and H. L. Anderson, Nanoscale, 2019, 11, 14820-14827.

76 C. Thiele, H. Vieker, A. Beyer, B. S. Flavel, F. Hennrich, D. M. Torres, T. R. Eaton, M. Mayor, M. M. Kappes, A. Gölzhäuser, H. v. Löhneysen and R. Krupke, Appl. Phys. Lett., 2014, 104, 103102.

77 C. W. Marquardt, S. Grunder, A. Błaszczyk, S. Dehm, F. Hennrich, H. v. Löhneysen, M. Mayor and R. Krupke, Nat. Nanotechnol., 2010, 5, 863-867.

78 M. L. Kirk, R. Dangi, D. Habel-Rodriguez, J. Yang, D. A. Shultz and J. Zhang, Chem. Sci., 2020, 11, 1142511434.

79 K. Pettersson, J. Wiberg, T. Ljungdahl, J. Mårtensson and B. Albinsson, J. Phys. Chem. A, 2006, 110, 319-326.

80 C. Atienza, N. Martín, M. Wielopolski, N. Haworth, T. Clark and D. M. Guldi, Chem. Commun., 2006, 32023204.

81 K. E. Linton, M. A. Fox, L.-O. Pålsson and M. R. Bryce, Chem. - Eur. J., 2015, 21, 3997-4007.

82 P. J. Low, Coord. Chem. Rev., 2013, 257, 1507-1532.

83 M. A. Fox, B. Le Guennic, R. L. Roberts, D. A. Brue, D. S. Yufit, J. A. K. Howard, G. Manca, J.-F. Halet, F. Hartl and P. J. Low, J. Am. Chem. Soc., 2011, 133, 18433-18446.

84 P. Wautelet, J. Le Moigne, V. Videva and P. Turek, J. Org. Chem., 2003, 68, 8025-8036.

85 P. Reddy, S.-Y. Jang, R. A. Segalman and A. Majumdar, Science, 2007, 315, 1568-1571.

86 J. R. Widawsky, P. Darancet, J. B. Neaton and L. Venkataraman, Nano Lett., 2012, 12, 354-358.

87 M. Tsutsui, T. Morikawa, Y. He, A. Arima and M. Taniguchi, Sci. Rep., 2015, 5, 11519.

88 L. Cui, S. Hur, Z. A. Akbar, J. C. Klöckner, W. Jeong, F. Pauly, S.-Y. Jang, P. Reddy and E. Meyhofer, Nature, 2019, 572, 628-633.

89 N. Mosso, H. Sadeghi, A. Gemma, S. Sangtarash, U. Drechsler, C. Lambert and B. Gotsmann, Nano Lett., 2019, 19, 7614-7622.

90 A. Tan, S. Sadat and P. Reddy, Appl. Phys. Lett., 2010, 96, 013110.

91 S. Park and H. J. Yoon, Nano Lett., 2018, 18, 7715-7718.

92 F. G. Guijarro, S. Medina Rivero, S. Gunasekaran, I. Arretxea, R. Ponce Ortiz, R. Caballero, P. de la Cruz, F. Langa, L. Venkataraman and J. Casado, RSC Adv., 2020, 10, 41264-41271.

93 R. Huber, M. T. González, S. Wu, M. Langer, S. Grunder, V. Horhoiu, M. Mayor, M. R. Bryce, C. Wang, R. Jitchati, C. Schönenberger and M. Calame, J. Am. Chem. Soc., 2008, 130, 1080-1084.
94 S. Martin, W. Haiss, S. J. Higgins and R. J. Nichols, Nano Lett., 2010, 10, 2019-2023.

95 C. R. Arroyo, R. Frisenda, K. Moth-Poulsen, J. S. Seldenthuis, T. Bjørnholm and H. S. J. van der Zant, Nanoscale Res. Lett., 2013, 8, 234.

96 X. Zeng, C. Wang, A. S. Batsanov, M. R. Bryce, J. Gigon, B. Urasinska-Wojcik and G. J. Ashwell, J. Org. Chem., 2010, 75, 130-136.

97 Z. Xie, I. Bâldea, A. T. Demissie, C. E. Smith, Y. Wu, G. Haugstad and C. D. Frisbie, J. Am. Chem. Soc., 2017, 139, 5696-5699.

98 V. Kolivoška, J. Šebera, T. Sebechlebská, M. Lindner, J. Gasior, G. Mészáros, M. Mayor, M. Valášek and M. Hromadová, Chem. Commun., 2019, 55, 33513354.

99 B. Capozzi, E. J. Dell, T. C. Berkelbach, D. R. Reichman, L. Venkataraman and L. M. Campos, J. Am. Chem. Soc., 2014, 136, 10486-10492.

100 T. Ohto, T. Inoue, H. Stewart, Y. Numai, Y. Aso, Y. Ie, R. Yamada and H. Tada, J. Phys. Chem. Lett., 2019, 10, 5292-5296.

101 Y. Ie, Y. Okamoto, T. Inoue, T. Seo, T. Ohto, R. Yamada, H. Tada and Y. Aso, J. Am. Chem. Soc., 2021, 143, 599603.

102 P. Moreno-García, M. Gulcur, D. Z. Manrique, T. Pope, W. Hong, V. Kaliginedi, C. Huang, A. S. Batsanov, M. R. Bryce, C. Lambert and T. Wandlowski, J. Am. Chem. Soc., 2013, 135, 12228-12240.

103 D. C. Milan, O. A. Al-Owaedi, M.-C. Oerthel, S. MarquésGonzález, R. J. Brooke, M. R. Bryce, P. Cea, J. Ferrer, S. J. Higgins, C. J. Lambert, P. J. Low, D. Z. Manrique, S. Martin, R. J. Nichols, W. Schwarzacher and V. M. García-Suárez, J. Phys. Chem. C, 2016, 120, 1566615674.

104 Y. Zang, T. Fu, Q. Zou, F. Ng, H. Li, M. L. Steigerwald, C. Nuckolls and L. Venkataraman, Nano Lett., 2020, 20, 8415-8419.

105 E. Leary, B. Limburg, A. Alanazy, S. Sangtarash, I. Grace, K. Swada, L. J. Esdaile, M. Noori, M. T. González, G. Rubio-Bollinger, H. Sadeghi, A. Hodgson, N. Agraït, S. J. Higgins, C. J. Lambert, H. L. Anderson and R. J. Nichols, J. Am. Chem. Soc., 2018, 140, 12877-12883.

106 T. Hines, I. Diez-Perez, J. Hihath, H. Liu, Z.-S. Wang, J. Zhao, G. Zhou, K. Müllen and N. Tao, J. Am. Chem. Soc., 2010, 132, 11658-11664.

107 Z. Cai, W.-Y. Lo, T. Zheng, L. Li, N. Zhang, Y. Hu and L. Yu, J. Am. Chem. Soc., 2016, 138, 10630-10635.

108 R. Frisenda, D. Stefani and H. S. J. van der Zant, Acc. Chem. Res., 2018, 51, 1359-1367.

109 G. Sedghi, K. Sawada, L. J. Esdaile, M. Hoffmann, H. L. Anderson, D. Bethell, W. Haiss, S. J. Higgins and R. J. Nichols, J. Am. Chem. Soc., 2008, 130, 8582-8583.

110 A. Mishchenko, D. Vonlanthen, V. Meded, M. Bürkle, C. Li, I. V. Pobelov, A. Bagrets, J. K. Viljas, F. Pauly, F. Evers, M. Mayor and T. Wandlowski, Nano Lett., 2010, 10, 156-163. 
111 W. Haiss, C. Wang, I. Grace, A. S. Batsanov, D. J. Schiffrin, S. J. Higgins, M. R. Bryce, C. J. Lambert and R. J. Nichols, Nat. Mater., 2006, 5, 995-1002.

112 X. Zhao, C. Huang, M. Gulcur, A. S. Batsanov, M. Baghernejad, W. Hong, M. R. Bryce and T. Wandlowski, Chem. Mater., 2013, 25, 4340-4347.

113 R. Chinchilla and C. Nájera, Chem. Rev., 2007, 107, 874922.

114 R. Chinchilla and C. Nájera, Chem. Soc. Rev., 2011, 40, 5084-5121.

115 N. M. Jenny, M. Mayor and T. R. Eaton, Eur. J. Org. Chem., 2011, 4965-4983.

116 P. Zwick, C. Hsu, M. El Abbassi, O. Fuhr, D. Fenske, D. Dulić, H. S. J. van der Zant and M. Mayor, J. Org. Chem., 2020, 85, 15072-15081.

117 Y. Geng, S. Sangtarash, C. Huang, H. Sadeghi, Y. Fu, W. Hong, T. Wandlowski, S. Decurtins, C. J. Lambert and S.-X. Liu, J. Am. Chem. Soc., 2015, 137, 4469-4476.

118 Y. Yang, M. Gantenbein, A. Alqorashi, J. Wei, S. Sangtarash, D. Hu, H. Sadeghi, R. Zhang, J. Pi, L. Chen, X. Huang, R. Li, J. Liu, J. Shi, W. Hong, C. J. Lambert and M. R. Bryce, J. Phys. Chem. C, 2018, 122, 14965-14970.

119 G. Petrillo, M. Novi, G. Garbarino and M. Filiberti, Tetrahedron Lett., 1988, 29, 4185-4188.

120 D. T. Gryko, C. Clausen, K. M. Roth, N. Dontha, D. F. Bocian, W. G. Kuhr and J. S. Lindsey, J. Org. Chem., 2000, 65, 7345-7355.

121 C. Wang, A. S. Batsanov, M. R. Bryce and I. Sage, Synthesis, 2003, 2089-2095.

122 J. Liang, R. E. G. Smith, A. Vezzoli, L. Xie, D. C. Milan, R. Davidson, A. Beeby, P. J. Low, S. J. Higgins, B. Mao and R. J. Nichols, Electrochim. Acta, 2016, 220, 436-443.

123 S. Martín, I. Grace, M. R. Bryce, C. Wang, R. Jitchati, A. S. Batsanov, S. J. Higgins, C. J. Lambert and R. J. Nichols, J. Am. Chem. Soc., 2010, 132, 9157-9164.

124 H. Valkenier, E. H. Huisman, P. A. van Hal, D. M. de Leeuw, R. C. Chiechi and J. C. Hummelen, J. Am. Chem. Soc., 2011, 133, 4930-4939.

125 V. Kaliginedi, P. Moreno-García, H. Valkenier, W. Hong, V. M. García-Suárez, P. Buiter, J. L. H. Otten, J. C. Hummelen, C. J. Lambert and T. Wandlowski, J. Am. Chem. Soc., 2012, 134, 5262-5275.

126 X. Wang, T. L. R. Bennett, A. Ismael, L. A. Wilkinson, J. Hamill, A. J. P. White, I. M. Grace, O. V. Kolosov, T. Albrecht, B. J. Robinson, N. J. Long, L. F. Cohen and C. J. Lambert, J. Am. Chem. Soc., 2020, 142, 85558560 .

127 C. Wang, A. S. Batsanov and M. R. Bryce, J. Org. Chem., 2006, 71, 108-116.

128 Q. Lu, K. Liu, H. Zhang, Z. Du, X. Wang and F. Wang, ACS Nano, 2009, 3, 3861-3868.

129 G. J. Ashwell, B. Urasinska, C. Wang, M. R. Bryce, I. Grace and C. J. Lambert, Chem. Commun., 2006, 4706-4708.

130 C. Thiele, L. Gerhard, T. R. Eaton, D. M. Torres, M. Mayor, W. Wulfhekel, H. v. Löhneysen and M. Lukas, New J. Phys., 2015, 17, 053043.
131 D. Miguel, L. Álvarez de Cienfuegos, A. Martín-Lasanta, S. P. Morcillo, L. A. Zotti, E. Leary, M. Bürkle, Y. Asai, R. Jurado, D. J. Cárdenas, G. Rubio-Bollinger, N. Agraït, J. M. Cuerva and M. T. González, J. Am. Chem. Soc., 2015, 137, 13818-13826.

132 X. Liu, S. Sangtarash, D. Reber, D. Zhang, H. Sadeghi, J. Shi, Z.-Y. Xiao, W. Hong, C. J. Lambert and S.-X. Liu, Angew. Chem., Int. Ed., 2017, 56, 173-176.

133 W. Chen, H. Li, J. R. Widawsky, C. Appayee, L. Venkataraman and R. Breslow, J. Am. Chem. Soc., 2014, 136, 918-920.

134 Y.-H. Wang, F. Yan, D.-F. Li, Y.-F. Xi, R. Cao, J.-F. Zheng, Y. Shao, S. Jin, J.-Z. Chen and X.-S. Zhou, J. Phys. Chem. Lett., 2021, 12, 758-763.

135 L.-J. Wang, A. Yong, K.-G. Zhou, L. Tan, J. Ye, G.-P. Wu, Z.-G. Xu and H.-L. Zhang, Chem. - Asian J., 2013, 8, 19011909.

136 M. Gantenbein, X. Li, S. Sangtarash, J. Bai, G. Olsen, A. Alqorashi, W. Hong, C. J. Lambert and M. R. Bryce, Nanoscale, 2019, 11, 20659-20666.

137 M. Gantenbein, L. Wang, A. A. Al-jobory, A. K. Ismael, C. J. Lambert, W. Hong and M. R. Bryce, Sci. Rep., 2017, 7, 1794.

138 A. Alanazy, E. Leary, T. Kobatake, S. Sangtarash, M. T. González, H.-W. Jiang, G. R. Bollinger, N. Agräit, H. Sadeghi, I. Grace, S. J. Higgins, H. L. Anderson, R. J. Nichols and C. J. Lambert, Nanoscale, 2019, 11, 13720-13724.

139 A. Ismael, X. Wang, T. L. R. Bennett, L. A. Wilkinson, B. J. Robinson, N. J. Long, L. F. Cohen and C. J. Lambert, Chem. Sci., 2020, 11, 6836-6841.

140 M. Famili, C. Jia, X. Liu, P. Wang, I. M. Grace, J. Guo, Y. Liu, Z. Feng, Y. Wang, Z. Zhao, S. Decurtins, R. Häner, Y. Huang, S.-X. Liu, C. J. Lambert and X. Duan, Chem, 2019, 5, 474-484.

141 F. Schwarz, M. Koch, G. Kastlunger, H. Berke, R. Stadler, K. Venkatesan and E. Lörtscher, Angew. Chem., Int. Ed., 2016, 55, 11781-11786.

142 C. Huang, S. Chen, K. Baruël Ørnsø, D. Reber, M. Baghernejad, Y. Fu, T. Wandlowski, S. Decurtins, W. Hong, K. S. Thygesen and S.-X. Liu, Angew. Chem., Int. Ed., 2015, 54, 14304-14307.

143 Z. Li, H. Li, S. Chen, T. Froehlich, C. Yi, C. Schönenberger, M. Calame, S. Decurtins, S.-X. Liu and E. Borguet, J. Am. Chem. Soc., 2014, 136, 8867-8870.

144 Z. Li, T.-H. Park, J. Rawson, M. J. Therien and E. Borguet, Nano Lett., 2012, 12, 2722-2727.

145 Z. Li, M. Smeu, T.-H. Park, J. Rawson, Y. Xing, M. J. Therien, M. A. Ratner and E. Borguet, Nano Lett., 2014, 14, 5493-5499.

146 M. El Abbassi, P. Zwick, A. Rates, D. Stefani, A. Prescimone, M. Mayor, H. S. J. van der Zant and D. Dulić, Chem. Sci., 2019, 10, 8299-8305.

147 E. Koyama, Y. Naitoh, H. Tokuhisa, T. Nakamura, M. Horikawa, T. Ishida, K. Fujiwara, W. Mizutani, Y. Nagawa and M. Kanesato, Jpn. J. Appl. Phys., 2008, 47, 7369-7371. 
148 J. Ponce, C. R. Arroyo, S. Tatay, R. Frisenda, P. Gaviña, D. Aravena, E. Ruiz, H. S. J. van der Zant and E. Coronado, J. Am. Chem. Soc., 2014, 136, 8314-8322.

149 T. Brandl, M. El Abbassi, D. Stefani, R. Frisenda, G. D. Harzmann, H. S. J. van der Zant and M. Mayor, Eur. J. Org. Chem., 2019, 5334-5343.

150 M. Kiguchi, H. Nakamura, Y. Takahashi, T. Takahashi and T. Ohto, J. Phys. Chem. C, 2010, 114, 22254-22261.

151 G. Yang, H. Wu, J. Wei, J. Zheng, Z. Chen, J. Liu, J. Shi, Y. Yang and W. Hong, Chin. Chem. Lett., 2018, 29, 147-150.

152 R. Miao, H. Xu, M. Skripnik, L. Cui, K. Wang, K. G. L. Pedersen, M. Leijnse, F. Pauly, K. Wärnmark, E. Meyhofer, P. Reddy and H. Linke, Nano Lett., 2018, 18, 5666-5672.

153 F. Jiang, D. I. Trupp, N. Algethami, H. Zheng, W. He, A. Alqorashi, C. Zhu, C. Tang, R. Li, J. Liu, H. Sadeghi, J. Shi, R. Davidson, M. Korb, A. N. Sobolev, M. Naher, S. Sangtarash, P. J. Low, W. Hong and C. J. Lambert, Angew. Chem., Int. Ed., 2019, 58, 18987-18993.

154 M. Mayor, H. B. Weber, J. Reichert, M. Elbing, C. von Hänisch, D. Beckmann and M. Fischer, Angew. Chem., Int. Ed., 2003, 42, 5834-5838.

155 D. Z. Manrique, C. Huang, M. Baghernejad, X. Zhao, O. A. Al-Owaedi, H. Sadeghi, V. Kaliginedi, W. Hong, M. Gulcur, T. Wandlowski, M. R. Bryce and C. J. Lambert, Nat. Commun., 2015, 6, 6389.

156 C. R. Arroyo, S. Tarkuc, R. Frisenda, J. S. Seldenthuis, C. H. M. Woerde, R. Eelkema, F. C. Grozema and H. S. J. van der Zant, Angew. Chem., Int. Ed., 2013, 52, 3152-3155.

157 B. Huang, X. Liu, Y. Yuan, Z.-W. Hong, J.-F. Zheng, L.-Q. Pei, Y. Shao, J.-F. Li, X.-S. Zhou, J.-Z. Chen, S. Jin and B.-W. Mao, J. Am. Chem. Soc., 2018, 140, 17685-17690.

158 E. Leary, A. La Rosa, M. T. González, G. Rubio-Bollinger, N. Agraït and N. Martín, Chem. Soc. Rev., 2015, 44, 920942.

159 S. Wu, M. T. González, R. Huber, S. Grunder, M. Mayor, C. Schönenberger and M. Calame, Nat. Nanotechnol., 2008, 3, 569-574.

160 W. Hong, D. Z. Manrique, P. Moreno-García, M. Gulcur, A. Mishchenko, C. J. Lambert, M. R. Bryce and T. Wandlowski, J. Am. Chem. Soc., 2012, 134, 2292-2304.

161 R. Frisenda, S. Tarkuç, E. Galán, M. L. Perrin, R. Eelkema, F. C. Grozema and H. S. J. van der Zant, Beilstein J. Nanotechnol., 2015, 6, 1558-1567.

162 K. Yoshida, I. V. Pobelov, D. Z. Manrique, T. Pope, G. Mészáros, M. Gulcur, M. R. Bryce, C. J. Lambert and T. Wandlowski, Sci. Rep., 2015, 5, 9002.

163 L. A. Zotti, T. Kirchner, J.-C. Cuevas, F. Pauly, T. Huhn, E. Scheer and A. Erbe, Small, 2010, 6, 1529-1535.

164 Y. S. Park, J. R. Widawsky, M. Kamenetska, M. L. Steigerwald, M. S. Hybertsen, C. Nuckolls and L. Venkataraman, J. Am. Chem. Soc., 2009, 131, 1082010821.

165 I. J. Planje, R. J. Davidson, A. Vezzoli, A. Daaoub, S. Sangtarash, H. Sadeghi, S. Martín, P. Cea,
C. J. Lambert, A. Beeby, S. J. Higgins and R. J. Nichols, ACS Sens., 2021, 6, 530-537.

166 M. Naher, D. C. Milan, O. A. Al-Owaedi, I. J. Planje, S. Bock, J. Hurtado-Gallego, P. Bastante, Z. M. Abd Dawood, L. Rincón-García, G. Rubio-Bollinger, S. J. Higgins, N. Agraït, C. J. Lambert, R. J. Nichols and P. J. Low, J. Am. Chem. Soc., 2021, 143, 3817-3829.

167 S. Bock, O. A. Al-Owaedi, S. G. Eaves, D. C. Milan, M. Lemmer, B. W. Skelton, H. M. Osorio, R. J. Nichols, S. J. Higgins, P. Cea, N. J. Long, T. Albrecht, S. Martín, C. J. Lambert and P. J. Low, Chem. - Eur. J., 2017, 23, 2133-2143.

168 E. Lörtscher, C. J. Cho, M. Mayor, M. Tschudy, C. Rettner and H. Riel, ChemPhysChem, 2011, 12, 1677-1682.

169 J. J. Stapleton, T. A. Daniel, S. Uppili, O. M. Cabarcos, J. Naciri, R. Shashidhar and D. L. Allara, Langmuir, 2005, 21, 11061-11070.

170 R. R. Ferradás, S. Marqués-González, H. M. Osorio, J. Ferrer, P. Cea, D. C. Milan, A. Vezzoli, S. J. Higgins, R. J. Nichols, P. J. Low, V. M. García-Suárez and S. Martín, RSC Adv., 2016, 6, 75111-75121.

171 S. Marqués-González, D. S. Yufit, J. A. K. Howard, S. Martín, H. M. Osorio, V. M. García-Suárez, R. J. Nichols, S. J. Higgins, P. Cea and P. J. Low, Dalton Trans., 2013, 42, 338-341.

172 W. Hong, H. Li, S.-X. Liu, Y. Fu, J. Li, V. Kaliginedi, S. Decurtins and T. Wandlowski, J. Am. Chem. Soc., 2012, 134, 19425-19431.

173 M. Baghernejad, Y. Yang, O. A. Al-Owaedi, Y. Aeschi, B.-F. Zeng, Z. M. Abd Dawood, X. Li, J. Liu, J. Shi, S. Decurtins, S.-X. Liu, W. Hong and C. J. Lambert, Chem. - Eur. J., 2020, 26, 5264-5269.

174 P. Pla-Vilanova, A. C. Aragonès, S. Ciampi, F. Sanz, N. Darwish and I. Diez-Perez, Nanotechnology, 2015, 26, 381001.

175 L. Herrer, A. González-Orive, S. Marqués-González, S. Martín, R. J. Nichols, J. L. Serrano, P. J. Low and P. Cea, Nanoscale, 2019, 11, 7976-7985.

176 Y. Xing, T.-H. Park, R. Venkatramani, S. Keinan, D. N. Beratan, M. J. Therien and E. Borguet, J. Am. Chem. Soc., 2010, 132, 7946-7956.

177 I. L. Herrer, A. K. Ismael, D. C. Milán, A. Vezzoli, S. Martín, A. González-Orive, I. Grace, C. Lambert, J. L. Serrano, R. J. Nichols and P. Cea, J. Phys. Chem. Lett., 2018, 9, 5364-5372.

178 J. M. Hamill, X. T. Zhao, G. Mészáros, M. R. Bryce and M. Arenz, Phys. Rev. Lett., 2018, 120, 016601.

179 M. A. Karimi, S. G. Bahoosh, M. Valášek, M. Bürkle, M. Mayor, F. Pauly and E. Scheer, Nanoscale, 2016, 8, 10582-10590.

180 L. Gerhard, K. Edelmann, J. Homberg, M. Valášek, S. G. Bahoosh, M. Lukas, F. Pauly, M. Mayor and W. Wulfhekel, Nat. Commun., 2017, 8, 14672.

181 J. Šebera, V. Kolivoška, M. Valášek, J. Gasior, R. Sokolová, G. Mészáros, W. Hong, M. Mayor and M. Hromadová, J. Phys. Chem. C, 2017, 121, 12885-12894. 
182 Y. Ie, T. Hirose, H. Nakamura, M. Kiguchi, N. Takagi, M. Kawai and Y. Aso, J. Am. Chem. Soc., 2011, 133, 30143022.

183 E. Escorihuela, P. Cea, S. Bock, D. C. Milan, S. Naghibi, H. M. Osorio, R. J. Nichols, P. J. Low and S. Martin, J. Mater. Chem. C, 2020, 8, 672-682.

184 L. Herrer, A. Ismael, S. Martín, D. C. Milan, J. L. Serrano, R. J. Nichols, C. Lambert and P. Cea, Nanoscale, 2019, 11, 15871-15880.

185 L. C. Chen, J. Zheng, J. Liu, X. T. Gong, Z. Z. Chen, R. X. Guo, X. Huang, Y. P. Zhang, L. Zhang, R. Li, X. Shao, W. Hong and H. L. Zhang, Small, 2020, 16, e2002808.

186 C. A. Martin, D. Ding, J. K. Sørensen, T. Bjørnholm, J. M. van Ruitenbeek and H. S. J. van der Zant, J. Am. Chem. Soc., 2008, 130, 13198-13199.

187 M. T. González, E. Leary, R. García, P. Verma, M. Á. Herranz, G. Rubio-Bollinger, N. Martín and N. Agraït, J. Phys. Chem. C, 2011, 115, 17973-17978.

188 A. K. Mahapatro, J. Ying, T. Ren and D. B. Janes, Nano Lett., 2008, 8, 2131-2136.

189 L. M. Ballesteros, S. Martín, J. Cortés, S. MarquésGonzález, S. J. Higgins, R. J. Nichols, P. J. Low and P. Cea, Chem. - Eur. J., 2013, 19, 5352-5363.

190 C. Tang, J. Zheng, Y. Ye, J. Liu, L. Chen, Z. Yan, Z. Chen, L. Chen, X. Huang, J. Bai, Z. Chen, J. Shi, H. Xia and W. Hong, iScience, 2020, 23, 100770.

191 Y. Fu, S. Chen, A. Kuzume, A. Rudnev, C. Huang, V. Kaliginedi, M. Baghernejad, W. Hong, T. Wandlowski, S. Decurtins and S.-X. Liu, Nat. Commun., 2015, 6, 6403.

192 L. M. Ballesteros, S. Martín, J. Cortés, S. MarquésGonzález, F. Pérez-Murano, R. J. Nichols, P. J. Low and P. Cea, Adv. Mater. Interfaces, 2014, 1, 1400128.

193 R. Ezquerra, S. G. Eaves, S. Bock, B. W. Skelton, F. PérezMurano, P. Cea, S. Martín and P. J. Low, J. Mater. Chem. C, 2019, 7, 6630-6640.

194 C. R. Parker, Z. Wei, C. R. Arroyo, K. Jennum, T. Li, M. Santella, N. Bovet, G. Zhao, W. Hu, H. S. J. van der Zant, M. Vanin, G. C. Solomon, B. W. Laursen, K. Nørgaard and M. B. Nielsen, Adv. Mater., 2013, 25, 405409.

195 C. R. Parker, E. Leary, R. Frisenda, Z. Wei, K. S. Jennum, E. Glibstrup, P. B. Abrahamsen, M. Santella, M. A. Christensen, E. A. Della Pia, T. Li, M. T. Gonzalez, X. Jiang, T. J. Morsing, G. Rubio-Bollinger, B. W. Laursen, K. Nørgaard, H. van der Zant, N. Agrait and M. B. Nielsen, J. Am. Chem. Soc., 2014, 136, 16497-16507.

196 M. Valášek, M. Lindner and M. Mayor, Beilstein J. Nanotechnol., 2016, 7, 374-405.

197 T. Gu and J.-F. Nierengarten, Tetrahedron Lett., 2001, 42, 3175-3178.

198 Y. Shirai, Y. Zhao, L. Cheng and J. M. Tour, Org. Lett., 2004, 6, 2129-2132.

199 E. Leary, M. T. González, C. van der Pol, M. R. Bryce, S. Filippone, N. Martín, G. Rubio-Bollinger and N. Agraït, Nano Lett., 2011, 11, 2236-2241.
200 X. Guo, J. P. Small, J. E. Klare, Y. Wang, M. S. Purewal, I. W. Tam, B. H. Hong, R. Caldwell, L. Huang, S. Brien, J. Yan, R. Breslow, S. J. Wind, J. Hone, P. Kim and C. Nuckolls, Science, 2006, 311, 356.

201 M. T. González, X. Zhao, D. Z. Manrique, D. Miguel, E. Leary, M. Gulcur, A. S. Batsanov, G. Rubio-Bollinger, C. J. Lambert, M. R. Bryce and N. Agraït, J. Phys. Chem. C, 2014, 118, 21655-21662.

202 H. Dekkiche, A. Gemma, F. Tabatabaei, A. S. Batsanov, T. Niehaus, B. Gotsmann and M. R. Bryce, Nanoscale, 2020, 12, 18908-18917.

203 K. Baheti, J. A. Malen, P. Doak, P. Reddy, S.-Y. Jang, T. D. Tilley, A. Majumdar and R. A. Segalman, Nano Lett., 2008, 8, 715-719.

204 J. Chen, W. Wang, M. A. Reed, A. M. Rawlett, D. W. Price and J. M. Tour, Appl. Phys. Lett., 2000, 77, 1224-1226.

205 J. J. Stapleton, P. Harder, T. A. Daniel, M. D. Reinard, Y. Yao, D. W. Price, J. M. Tour and D. L. Allara, Langmuir, 2003, 19, 8245-8255.

206 L.-J. Wang, K.-G. Zhou, L. Tan, H. Wang, Z.-F. Shi, G.-P. Wu, Z.-G. Xu, X.-P. Cao, H.-X. He and H.-L. Zhang, Chem. - Eur. J., 2011, 17, 8414-8423.

207 H. Masai, T. Fujihara, Y. Tsuji and J. Terao, Chem. - Eur. J., 2017, 23, 15073-15079.

208 J. Inatomi, S. Fujii, S. Marqués-González, H. Masai, Y. Tsuji, J. Terao and M. Kiguchi, J. Phys. Chem. C, 2015, 119, 19452-19457.

209 M. Kiguchi, S. Nakashima, T. Tada, S. Watanabe, S. Tsuda, Y. Tsuji and J. Terao, Small, 2012, 8, 726-730.

210 H. Masai, J. Terao, S. Seki, S. Nakashima, M. Kiguchi, K. Okoshi, T. Fujihara and Y. Tsuji, J. Am. Chem. Soc., 2014, 136, 1742-1745.

211 J. Fock, M. Leijnse, K. Jennum, A. S. Zyazin, J. Paaske, P. Hedegård, M. B. Nielsen and H. S. J. van der Zant, Phys. Rev. B: Condens. Matter Mater. Phys., 2012, 86, 235403.

212 X. Liu, X. Li, S. Sangtarash, H. Sadeghi, S. Decurtins, R. Häner, W. Hong, C. J. Lambert and S.-X. Liu, Nanoscale, 2018, 10, 18131-18134.

213 F. Yan, F. Chen, X.-H. Wu, J. Luo, X.-S. Zhou, J. R. Horsley, A. D. Abell, J. Yu, S. Jin and B.-W. Mao, J. Phys. Chem. C, 2020, 124, 8496-8503.

214 H. Valkenier, C. M. Guédon, T. Markussen, K. S. Thygesen, S. J. van der Molen and J. C. Hummelen, Phys. Chem. Chem. Phys., 2014, 16, 653-662.

215 M. Baghernejad, X. Zhao, K. B. Ørnsø, M. Füeg, P. Moreno-García, A. V. Rudnev, V. Kaliginedi, S. Vesztergom, C. Huang, W. Hong, P. Broekmann, T. Wandlowski, K. S. Thygesen and M. R. Bryce, J. Am. Chem. Soc., 2014, 136, 17922-17925.

216 R. García, M. Á. Herranz, E. Leary, M. T. González, G. R. Bollinger, M. Bürkle, L. A. Zotti, Y. Asai, F. Pauly, J. C. Cuevas, N. Agraït and N. Martín, Beilstein J. Org. Chem., 2015, 11, 1068-1078.

217 M. Koole, J. M. Thijssen, H. Valkenier, J. C. Hummelen and H. S. J. van der Zant, Nano Lett., 2015, 15, 5569-5573. 
218 F. Giacalone, M. Á. Herranz, L. Grüter, M. T. González, M. Calame, C. Schönenberger, C. R. Arroyo, G. RubioBollinger, M. Vélez, N. Agraït and N. Martín, Chem. Commun., 2007, 4854-4856.

219 J. Liu, X. Zhao, Q. Al-Galiby, X. Huang, J. Zheng, R. Li, C. Huang, Y. Yang, J. Shi, D. Z. Manrique, C. J. Lambert, M. R. Bryce and W. Hong, Angew. Chem., Int. Ed., 2017, 56, 13061-13065.

220 Y. Li, M. Baghernejad, A.-G. Qusiy, D. Z. Manrique, G. Zhang, J. Hamill, Y. Fu, P. Broekmann, W. Hong, T. Wandlowski, D. Zhang and C. Lambert, Angew. Chem., Int. Ed., 2015, 54, 13586-13589.

221 V. Kolivoska, P. Moreno-García, V. Kaliginedi, W. Hong, M. Mayor, N. Weibel and T. Wandlowski, Electrochim. Acta, 2013, 110, 709-717.

222 Y. Kim, T. J. Hellmuth, D. Sysoiev, F. Pauly, T. Pietsch, J. Wolf, A. Erbe, T. Huhn, U. Groth, U. E. Steiner and E. Scheer, Nano Lett., 2012, 12, 3736-3742.

223 D. Kim, H. Jeong, W.-T. Hwang, Y. Jang, D. Sysoiev, E. Scheer, T. Huhn, M. Min, H. Lee and T. Lee, Adv. Funct. Mater., 2015, 25, 5918-5923.

224 J. Koo, Y. Jang, L. Martin, D. Kim, H. Jeong, K. Kang, W. Lee, J. Kim, W.-T. Hwang, D. Xiang, E. Scheer, M. Kabdulov, T. Huhn, F. Pauly and T. Lee, ACS Appl. Mater. Interfaces, 2019, 11, 11645-11653.

225 H. B. Li, B. E. Tebikachew, C. Wiberg, K. Moth-Poulsen and J. Hihath, Angew. Chem., Int. Ed., 2020, 59, 1164111646.

226 C. Tang, Y. Tang, Y. Ye, Z. Yan, Z. Chen, L. Chen, L. Zhang, J. Liu, J. Shi, H. Xia and W. Hong, Chem, 2020, 6, 2770-2781.

227 D. Stefani, K. J. Weiland, M. Skripnik, C. Hsu, M. L. Perrin, M. Mayor, F. Pauly and H. S. J. van der Zant, Nano Lett., 2018, 18, 5981-5988.

228 P. Zwick, K. J. Weiland, J. Malinčík, D. Stefani, D. Häussinger, H. S. J. van der Zant, D. Dulić and M. Mayor, J. Org. Chem., 2020, 85, 118-128.

229 R. Frisenda, V. A. E. C. Janssen, F. C. Grozema, H. S. J. van der Zant and N. Renaud, Nat. Chem., 2016, 8, 1099-1104.

230 M. Schmidt, D. Wassy, M. Hermann, M. T. González, N. Agräit, L. A. Zotti, B. Esser and E. Leary, Chem. Commun., 2021, 57, 745-748.

231 D. C. Milan, A. Vezzoli, I. J. Planje and P. J. Low, Dalton Trans., 2018, 47, 14125-14138.

232 Q. Lu, C. Yao, X. Wang and F. Wang, J. Phys. Chem. C, 2012, 116, 17853-17861.

233 Y. Yuan, J.-F. Yan, D.-Q. Lin, B.-W. Mao and Y.-F. Yuan, Chem. - Eur. J., 2018, 24, 3545-3555.

234 F. Schwarz, G. Kastlunger, F. Lissel, C. Egler-Lucas, S. N. Semenov, K. Venkatesan, H. Berke, R. Stadler and E. Lörtscher, Nat. Nanotechnol., 2016, 11, 170-176.

235 O. A. Al-Owaedi, S. Bock, D. C. Milan, M.-C. Oerthel, M. S. Inkpen, D. S. Yufit, A. N. Sobolev, N. J. Long, T. Albrecht, S. J. Higgins, M. R. Bryce, R. J. Nichols, C. J. Lambert and P. J. Low, Nanoscale, 2017, 9, 99029912.
236 K. Liu, X. Wang and F. Wang, ACS Nano, 2008, 2, 23152323.

237 B. Kim, J. M. Beebe, C. Olivier, S. Rigaut, D. Touchard, J. G. Kushmerick, X. Y. Zhu and C. D. Frisbie, J. Phys. Chem. C, 2007, 111, 7521-7526.

238 F. Meng, Y.-M. Hervault, L. Norel, K. Costuas, C. Van Dyck, V. Geskin, J. Cornil, H. H. Hng, S. Rigaut and X. Chen, Chem. Sci., 2012, 3, 3113-3118.

239 R. Venkatramani, E. Wierzbinski, D. H. Waldeck and D. N. Beratan, Faraday Discuss., 2014, 174, 57-78.

240 K. Liu, G. Li, X. Wang and F. Wang, J. Phys. Chem. C, 2008, 112, 4342-4349.

241 J. Alqahtani, H. Sadeghi, S. Sangtarash and C. J. Lambert, Angew. Chem., Int. Ed., 2018, 57, 15065-15069.

242 Z. Tang, S. Hou, Q. Wu, Z. Tan, J. Zheng, R. Li, J. Liu, Y. Yang, H. Sadeghi, J. Shi, I. Grace, C. J. Lambert and W. Hong, Sci. Bull., 2020, 65, 944-950.

243 G. J. Snyder and A. H. Snyder, Energy Environ. Sci., 2017, 10, 2280-2283.

244 S. Sangtarash, H. Sadeghi and C. J. Lambert, Phys. Chem. Chem. Phys., 2018, 20, 9630-9637.

245 J. A. Malen, P. Doak, K. Baheti, T. D. Tilley, R. A. Segalman and A. Majumdar, Nano Lett., 2009, 9, 1164-1169.

246 H. Chen, S. Sangtarash, G. Li, M. Gantenbein, W. Cao, A. Alqorashi, J. Liu, C. Zhang, Y. Zhang, L. Chen, Y. Chen, G. Olsen, H. Sadeghi, M. R. Bryce, C. J. Lambert and W. Hong, Nanoscale, 2020, 12, 15150-15156.

247 S. K. Lee, T. Ohto, R. Yamada and H. Tada, Nano Lett., 2014, 14, 5276-5280.

248 J. C. Klöckner, J. C. Cuevas and F. Pauly, Phys. Rev. B, 2017, 96, 245419.

249 T. Markussen, J. Chem. Phys., 2013, 139, 244101.

250 S. Park, J. Jang, H. Kim, D. I. Park, K. Kim and H. J. Yoon, J. Mater. Chem. A, 2020, 8, 19746-19767.

251 H. Sadeghi, J. Phys. Chem. C, 2019, 123, 12556-12562.

252 M. Lemmer, M. S. Inkpen, K. Kornysheva, N. J. Long and T. Albrecht, Nat. Commun., 2016, 7, 12922.

253 D. Cabosart, M. E. Abbassi, D. Stefani, R. Frisenda, M. Calame, H. S. J. van der Zant and M. L. Perrin, Appl. Phys. Lett., 2019, 114, 143102.

254 A. Magyarkuti, N. Balogh, Z. Balogh, L. Venkataraman and A. Halbritter, Nanoscale, 2020, 12, 8355-8363.

255 F. Huang, R. Li, G. Wang, J. Zheng, Y. Tang, J. Liu, Y. Yang, Y. Yao, J. Shi and W. Hong, Phys. Chem. Chem. Phys., 2020, 22, 1674-1681.

256 M. El Abbassi, J. Overbeck, O. Braun, M. Calame, H. S. J. van der Zant and M. L. Perrin, Commun. Phys., 2021, 4, 50.

257 C. Yang, A. Qin, B. Z. Tang and X. Guo, J. Chem. Phys., 2020, 152, 120902.

258 S. Caneva, P. Gehring, V. M. García-Suárez, A. GarcíaFuente, D. Stefani, I. J. Olavarria-Contreras, J. Ferrer, C. Dekker and H. S. J. van der Zant, Nat. Nanotechnol., 2018, 13, 1126-1131.

259 Z. Tan, D. Zhang, H.-R. Tian, Q. Wu, S. Hou, J. Pi, H. Sadeghi, Z. Tang, Y. Yang, J. Liu, Y.-Z. Tan, Z.-B. Chen, 
J. Shi, Z. Xiao, C. Lambert, S.-Y. Xie and W. Hong, Nat. Commun., 2019, 10, 1748.

260 S. Zhao, Q. Wu, J. Pi, J. Liu, J. Zheng, S. Hou, J. Wei, R. Li, H. Sadeghi, Y. Yang, J. Shi, Z. Chen, Z. Xiao, C. Lambert and W. Hong, Sci. Adv., 2020, 6, eaba6714.

261 Y. Naitoh, Y. Tani, E. Koyama, T. Nakamura, T. Sumiya, T. Ogawa, G. Misawa, H. Shima, K. Sugawara, H. Suga and H. Akinaga, J. Phys. Chem. C, 2020, 124, 1400714015.

262 B.-F. Zeng, G. Wang, Q.-Z. Qian, Z.-X. Chen, X.-G. Zhang, Z.-X. Lu, S.-Q. Zhao, A.-N. Feng, J. Shi, Y. Yang and W. Hong, Small, 2020, 16, 2004720.

263 S. Sanvito, Chem. Soc. Rev., 2011, 40, 3336-3355.

264 A. R. Rocha, V. M. García-suárez, S. W. Bailey, C. J. Lambert, J. Ferrer and S. Sanvito, Nat. Mater., 2005, 4, 335-339.

265 C. Herrmann, G. C. Solomon and M. A. Ratner, J. Am. Chem. Soc., 2010, 132, 3682-3684.

266 R. Hayakawa, M. A. Karimi, J. Wolf, T. Huhn, M. S. Zöllner, C. Herrmann and E. Scheer, Nano Lett., 2016, 16, 4960-4967.

267 M. S. Zöllner, R. Nasri, H. Zhang and C. Herrmann, J. Phys. Chem. C, 2021, 125, 1208-1220.

268 J. Z. Low, G. Kladnik, L. L. Patera, S. Sokolov, G. Lovat, E. Kumarasamy, J. Repp, L. M. Campos, D. Cvetko, A. Morgante and L. Venkataraman, Nano Lett., 2019, 19, 2543-2548.
269 S. Delprat, M. Galbiati, S. Tatay, B. Quinard, C. Barraud, F. Petroff, P. Seneor and R. Mattana, J. Phys. D: Appl. Phys., 2018, 51, 473001.

270 S. Qiu, Y.-Y. Miao, G.-P. Zhang, J.-F. Ren, C.-K. Wang and G.-C. Hu, J. Phys. Chem. C, 2020, 124, 12144-12152.

271 R. J. Brooke, C. Jin, D. S. Szumski, R. J. Nichols, B.-W. Mao, K. S. Thygesen and W. Schwarzacher, Nano Lett., 2015, 15, 275-280.

272 A. N. Pal, D. Li, S. Sarkar, S. Chakrabarti, A. Vilan, L. Kronik, A. Smogunov and O. Tal, Nat. Commun., 2019, 10, 5565 .

273 M. Cinchetti, V. A. Dediu and L. E. Hueso, Nat. Mater., 2017, 16, 507-515.

274 G. D. Scott and D. Natelson, ACS Nano, 2010, 4, 3560-3579.

275 S. Wagner, F. Kisslinger, S. Ballmann, F. Schramm, R. Chandrasekar, T. Bodenstein, O. Fuhr, D. Secker, K. Fink, M. Ruben and H. B. Weber, Nat. Nanotechnol., 2013, 8, 575-579.

276 R. Frisenda, R. Gaudenzi, C. Franco, M. Mas-Torrent, C. Rovira, J. Veciana, I. Alcon, S. T. Bromley, E. Burzurí and H. S. J. van der Zant, Nano Lett., 2015, 15, 3109-3114.

277 W. Liang, M. P. Shores, M. Bockrath, J. R. Long and H. Park, Nature, 2002, 417, 725-729.

278 J. J. Parks, A. R. Champagne, T. A. Costi, W. W. Shum, A. N. Pasupathy, E. Neuscamman, S. Flores-Torres, P. S. Cornaglia, A. A. Aligia, C. A. Balseiro, G. K. L. Chan, H. D. Abruña and D. C. Ralph, Science, 2010, 328, 1370. 